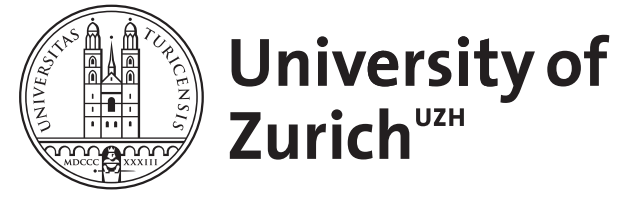

\title{
One-dimensional Chern-Simons theory
}

Alekseev, A ; Mnëv, P

\begin{abstract}
We study a one-dimensional toy version of the Chern-Simons theory. We construct its simplicial version which comprises features of a low-energy effective gauge theory and of a topological quantum field theory in the sense of Atiyah.
\end{abstract}

DOI: https://doi.org/10.1007/s00220-011-1290-1

Posted at the Zurich Open Repository and Archive, University of Zurich ZORA URL: https://doi.org/10.5167/uzh-71558

Journal Article

Published Version

Originally published at:

Alekseev, A; Mnëv, P (2011). One-dimensional Chern-Simons theory. Communications in Mathematical Physics, 307(1):185-227.

DOI: https://doi.org/10.1007/s00220-011-1290-1 


\title{
One-Dimensional Chern-Simons Theory
}

\author{
Anton Alekseev ${ }^{1}$, Pavel Mnëv ${ }^{2,3}$ \\ 1 Section of Mathematics, University of Geneva, 2-4 Rue du Lièvre, C.P. 64, 1211 Genève 4, Switzerland. \\ E-mail: Anton.Alekseev@unige.ch \\ 2 Petersburg Department of V. A. Steklov Institute of Mathematics, Fontanka 27, 191023 St. Petersburg, \\ Russia. E-mail: pmnev@pdmi.ras.ru \\ 3 Institut für Mathematik, Universität Zürich-Irchel, Winterthurerstrasse 190, CH-8057 Zürich, Switzerland
}

Received: 1 December 2010 / Accepted: 31 December 2010

Published online: 29 June 2011 - C Springer-Verlag 2011

\begin{abstract}
We study a one-dimensional toy version of the Chern-Simons theory. We construct its simplicial version which comprises features of a low-energy effective gauge theory and of a topological quantum field theory in the sense of Atiyah.
\end{abstract}

\section{Contents}

1. Introduction . . . . . . . . . . . . . . . . . 186

1.1 The logic of the paper . . . . . . . . . . . . . . . . . . . 187

1.2 The main results . . . . . . . . . . . . . . . . . . . . . . 188

1.3 A speculation: towards generalizations to topological quantum field theories in higher dimensions . . . . . . . . . . . . . . . . . . 188

1.4 Authorship . . . . . . . . . . . . . . . . . . . . . . . . 189

2. Simplicial Chern-Simons Theory on the Circle . . . . . . . . . . . . . 189

2.1 Continuum theory on the circle: fields, BV structure, action . . . . . . 190

2.2 Effective action on the cohomology of the circle . . . . . . . . . . . 192

2.2.1 Harmonic gauge. . . . . . . . . . . . . . . . . . . . . . . 192

2.2.2 Effective action on cohomology. . . . . . . . . . . . . . . 192

2.3 Simplicial Chern-Simons action on circle . . . . . . . . . . . 193

2.3.1 Cyclic Whitney gauge. . . . . . . . . . . . . . . . . . . 193

2.3.2 Chain homotopy, dressed chain homotopy. . . . . . . . . . . . . . 194

2.3.3 Simplicial action. . . . . . . . . . . . . . . . . . . . . . 197

2.3.4 Remarks. . . . . . . . . . . . . . . . . . . . . . . 198

3. Approach Through Operator Formalism . . . . . . . . . . . . . . . . 201

3.1 One-dimensional Chern-Simons theory in operator formalism . . . . . 202

3.1.1 First approximation. . . . . . . . . . . . . . . . 202

3.1.2 Imposing the cyclic Whitney gauge. . . . . . . . . . . . . . 202

3.1.3 Consistency check: the effective action on cohomology. . . . . . . 203

3.1.4 Consistency check: the case of mutually commuting $\left\{A_{k}\right\}$ and $\psi^{\prime}=0.205$ 
3.2 One-dimensional Chern-Simons with boundary . . . . . . . . 206

3.2.1 One-dimensional simplicial Chern-Simons in the operator formalism.

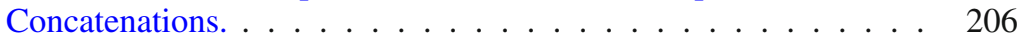

3.2.2 Simplicial aggregations. . . . . . . . . . . . . . . . . 207

3.2.3 Quantum master equation. . . . . . . . . . . . . . 210

4. Back to Path Integral . . . . . . . . . . . . . . . . . . . . . . . . . 212

4.1 Representation of $C l(\mathfrak{g})$, complex polarization of $\mathfrak{g}$. . . . . . . . . 212

4.2 One-dimensional Chern-Simons in terms of Atiyah-Segal's axioms . . 214

4.3 Integrating out the bulk fields . . . . . . . . . . . . . . 215

4.4 From operator formalism to path integral . . . . . . . . . . . . . . 217

4.4.1 Abelian one-dimensional Chern-Simons theory. . . . . . . . . . . 217

4.4.2 Path integral for the non-abelian one-dimensional Chern-Simons theory in the cyclic Whitney gauge. End of proof of Theorem 2. . . . 219

4.5 Simplicial action on an interval . . . . . . . . . . . . . 221

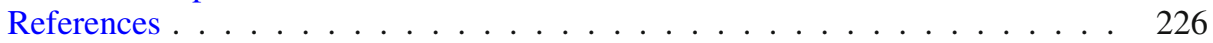

\section{Introduction}

We begin by considering a one-dimensional version of the Chern-Simons theory on a circle. This is a gauge theory in the Batalin-Vilkovisky formalism defined by the action

$$
S(A, \psi)=\frac{1}{2} \int(\psi, d \psi)+(\psi,[A, \psi]),
$$

where the field $\psi$ is an odd function on the circle with values in a quadratic Lie algebra $\mathfrak{g}$ (that is, $\mathfrak{g}$ has an invariant non-degenerate scalar product, e.g. the Killing form in the case of $\mathfrak{g}$ semisimple), and the field $A$ is an even 1 -form with values in $\mathfrak{g}$. We address the problem of constructing an effective BV action induced by a triangulation of the circle (that is, a splitting of the circle into a finite number of segments).

This problem is interesting by itself since it is related to discretization of differential geometry. In fact, the action (1) encodes the structure of a unimodular cyclic differential graded Lie algebra (DGLA) on $\mathfrak{g}$-valued differential forms on the circle (for details, see [7]). Then, the effective action is a generating function of a discretized (homotopy) version of this DGLA structure induced on the chains of a triangulation (which can be viewed as discrete analogs of differential forms).

Another motivation for studying the effective action in the simple model defined by action (1) is a hope to get a new insight for constructing a discrete version of the 3-dimensional Chern-Simons theory. Such a discrete Chern-Simons theory would allow defining quantum invariants of 3-manifolds as finite-dimensional integrals, and it would be compatible with the gauge symmetry (i.e. it would satisfy the Batalin-Vilkovisky quantum master equation); cf. Sect. 1.3.

We show that the effective action of the one-dimensional Chern-Simons theory on a triangulated circle is given by an explicit albeit somewhat cumbersome formula (42). It immediately raises a number of questions. Indeed, the result is expected to satisfy the quantum master equation (QME) and to be compatible with simplicial aggregations (merging several 1-simplices of the triangulation). How can we check this directly? Another desire is to represent the effective action in a "simplicially-local" form (that is, as a sum of contributions where each term depends only on two neighboring segments).

It turns out that answers to these questions come from the following construction. First, we give a new definition of the one-dimensional simplicial Chern-Simons theory 
using the operator formalism, i.e. in the language of Clifford algebras $C l(\mathfrak{g})$ (Sect. 3.2.1). The partition function for a simplicial complex is then an element of $C l(\mathfrak{g})^{\otimes i}$ (where $i$ is half the number of boundary points of the simplicial complex), and it is a product of local $\mathrm{Cl}(\mathfrak{g})$-valued expressions (61) for 1-simplices. In particular, for a triangulated circle the partition function is a scalar (depending on simplicial "bulk fields"). In Sect. 4.4.2, we establish the equivalence between the operator formalism and the path integral formalism of Sect. 2. In the operator formalism, the consistency with simplicial aggregations is checked in a straightforward manner (Sect. 3.2.2). The partition function $Z_{\mathcal{I}}$ on the interval $\mathcal{I}$ satisfies Eq. (68):

$$
\hbar \frac{\partial}{\partial \tilde{\psi}^{a}} \frac{\partial}{\partial A^{a}} Z_{\mathcal{I}}+\frac{1}{\hbar}\left[\frac{1}{6} f^{a b c} \hat{\psi}^{a} \hat{\psi}^{b} \hat{\psi}^{c}, Z_{\mathcal{I}}\right]_{C l(\mathfrak{g})}=0
$$

which is a version of the quantum master equation (QME) adjusted for the presence of the boundary. It immediately implies the QME with boundary contributions for an arbitrary one-dimensional simplicial complex (73), and the usual QME for the triangulated circle (44).

In order to formulate the one-dimensional simplicial Chern-Simons theory in the spirit of Atiyah's axioms of TQFT (Sect. 4.2), we choose a complex polarization of $\mathfrak{g}$ :

$$
\mathfrak{g}_{\mathbb{C}}=\mathfrak{h} \oplus \overline{\mathfrak{h}} .
$$

This is always possible if $\mathfrak{g}$ is even-dimensional. Note however that in this way we break the original $O(\mathfrak{g})$-symmetry of the problem. The (compexified) Clifford algebra $C l\left(\mathfrak{g}_{\mathbb{C}}\right)$ is isomorphic to the matrix (super-)algebra $\operatorname{End}(\wedge \mathfrak{h})=\operatorname{End}(\operatorname{Fun}(\Pi \mathfrak{h}))$. Therefore, the space of states associated to a point in the one-dimensional Chern-Simons theory is $\mathcal{H}_{p t}=\operatorname{Fun}(\Pi \mathfrak{h})-$ the super vector space of polynomials in $(\operatorname{dim} \mathfrak{g}) / 2$ odd variables. The super-space $\mathcal{H}_{p t}$ is endowed with an odd third-order differential operator $\delta$. One-dimensional "cobordisms" are now equipped with triangulations. To a triangulated cobordism $\Theta$ we associate the "space of bulk fields" $\mathcal{F}_{\Theta}^{\text {bulk }}$, equipped with the BV Laplacian $\Delta_{\Theta}^{\text {bulk }}$. The partition function for a triangulated cobordism satisfies the quantum master equation (82). In addition to the operations of gluing and disjoint union (which are standard in Atiyah's picture), simplicial aggregations are allowed for triangulated cobordisms. The original continuum theory can be thought of as the simplicial theory in the limit of dense triangulation.

Matrix elements of the partition function for a triangulated cobordism can be written as path integrals for the one-dimensional Chern-Simons theory with BV gauge fixing in the bulk and holomorphic-antiholomorphic boundary conditions (Sect. 4.4.2). This brings us back to the formalism of effective BV actions. The action for an interval is given by a Gaussian integral, and one can compute it explicitly (see Eq. (117)).

1.1. The logic of the paper. In Sect. 2, we construct an effective BV action for the one-dimensional Chern-Simons theory on a triangulated circle. Section 2.2 contains a warm-up calculation of the effective BV action induced on the de Rham cohomology of the circle. In Sects. 2.3.1 and 2.3.2, we handle the gauge fixing in the path integral. The final result of Sect. 2 is the explicit formula (42) for the simplicial action on a triangulated circle (Theorem 1).

In Sect. 3, we address the Chern-Simons theory on a circle via the quantum mechanical operator formalism. The resulting effective BV action on a triangulation is given by formulae $(51,52)$. The comparison with the path integral formalism (Theorem 2 ) is 
postponed until Sect. 4.4.2. Two quick consistency checks of Theorem 2 are performed in Sect. 3.1.3 and Sect. 3.1.4. In Sect. 3.2.1, we explain how the operator formalism helps to give an Atiyah-style axiomatic formulation of one-dimensional simplicial Chern-Simons theory. In more detail, in this approach one associates operator-valued effective actions to triangulated one-dimensional cobordisms. This picture comprises the features of an Atiyah TQFT (concatenation of cobordisms is sent to the composition of operators) and of a simplicial theory (simplicial aggregations are sent to certain BV integrals for the effective actions, cf. Sect. 3.2.2). In Sect. 3.2.3, we prove that a version of the BatalinVilkovisky quantum master equation (QME) is fulfilled for the partition function of a triangulated 1-cobordism (Theorem 3). This implies the usual QME for the simplicial action (42) on the triangulated circle (see Corollary 1).

In Sect. 4, we prove Theorem 2. The proof follows the standard route of using the heat kernel expansion of the evolution operator in order to recover the path integral representation (Sect. 4.4). Along the way, we identify the space of states associated to a point in the one-dimensional Chern-Simons theory (Sect. 4.2). In order to do that (and to define integral kernels of evolution operators) one needs to introduce a complex polarization of the gauge Lie algebra $\mathfrak{g}$ (Sect. 4.1). In Sect. 4.5, we compute the Chern-Simons partition function for an interval by perturbative techniques (Proposition 4).

\subsection{The main results.}

- Theorem 1: an explicit formula (42) for the effective BV action of the one-dimensional Chern-Simons theory on a circle induced on cochains of a triangulation of the circle. An essential part of this result is the unique gauge fixing in the path integral given in Sect. 2.3.1.

- Expressions $(51,52)$ for the exponential of the simplicial action on the triangulated circle in terms of the Clifford algebra $C l(\mathfrak{g})$, and Theorem 2 giving a comparison with (42).

- Construction of the one-dimensional Chern-Simons theory as an Atiyah-style TQFT defined on triangulated 1-cobordisms. It comprises features of an Atiyah TQFT (concatenations), of a simplicial theory (simplicial aggregations) and of a Lagrangian gauge theory (quantum master equation). The partition function of this theory on a triangulated circle is the exponential of simplicial action considered in Sect. 2. The construction is presented in Sects. 3.2.1, 4.2.

- Theorem 3: the quantum master equation with boundary terms.

1.3. A speculation: towards generalizations to topological quantum field theories in higher dimensions. Our discussion of the one-dimensional Chern-Simons theory in Sect. 4.2 inspires a hope that a hybrid Atiyah-Lagrange picture can apply in other topological quantum field theories, e.g. in more general AKSZ theories (which include the 3-dimensional Chern-Simons theory and the 2-dimensional Poisson sigma model). This is the subject of the work in progress by A. Cattaneo, P. M. and N. Reshetikhin [8].

By the loose term "hybrid Atiyah-Lagrange picture" we mean a functor which associates to a $D$-cobordism $B_{\text {in }} \stackrel{\Sigma}{\rightarrow} B_{\text {out }}$ a partition function

$$
Z_{\Sigma} \in \mathcal{H}_{B_{\text {in }}}^{*} \otimes \mathcal{H}_{B_{\text {out }}} \otimes \operatorname{Fun}\left(\mathcal{F}_{\Sigma}^{\text {bulk }}\right) .
$$

It is a function on the "space of bulk fields" $\mathcal{F}_{\Sigma}^{\text {bulk }}$ (a finite-dimensional BV manifold) associated to the cobordism, and it takes values in linear operators from the space 
of states associated to incoming boundary $\mathcal{H}_{B_{\text {in }}}$ to the space of states associated to the outgoing boundary $\mathcal{H}_{B_{\text {out }}}$. Gluing of two cobordisms along the common boundary should be mapped to the composition of operators (as in Atiyah's picture). Gauge symmetry should be present in the form of a quantum master equation with boundary terms,

$$
\left(\hbar \Delta_{\Sigma}^{\text {bulk }}-\hbar^{-1} \delta_{B_{\text {in }}}^{*}+\hbar^{-1} \delta_{B_{\text {out }}}\right) Z_{\Sigma}=0
$$

cf. (82). Here $\Delta_{\Sigma}^{\text {bulk }}$ is the BV Laplacian on $\operatorname{Fun}\left(\mathcal{F}_{\Sigma}^{\text {bulk }}\right)$ and $\delta_{B}$ is a certain coboundary operator on the space of states $\mathcal{H}_{B}$ (the coboundary property $\delta_{B}^{2}=0$ might get spoiled by an anomaly, cf. (83)). Thus, a TQFT in the Atiyah-Lagrange picture would have features of both Atiyah's TQFT and of an effective gauge theory in the Lagrangian Batalin-Vilkovisky formalism.

One important example of an Atiyah-Lagrange TQFT would be an effective theory on zero modes of a continuum TQFT in Lagrangian formalism, with matrix elements of the partition function given by perturbative path integrals.

A more ambitious task would be to construct a discrete TQFT in the Atiyah-Lagrange picture, where cobordisms and boundary components carry "discretization data" (e.g. triangulations). Then, spaces of bulk fields and spaces of states depend on the discretization data, and aggregations of discretization data are mapped to finite-dimensional fiber BV integrals at the level of partition functions. One hopes to construct such discrete TQFTs from continuum ones by perturbative path integrals (this would be the higher-dimensional version of the integrals (104), (105)).

For an AKSZ sigma model on $\operatorname{Maps}(\bullet, \mathcal{M})$, our study gives an educated guess for the space of states $\mathcal{H}_{B}$ and for the coboundary operator $\delta_{B}$ appearing in the quantum master equation. In more detail, the space of states $\mathcal{H}_{B}$ should be the geometric quantization of the symplectic supermanifold $\Phi_{B}=\operatorname{Maps}(B, \mathcal{M})$ (the phase super-space). The AKSZ construction endows $\Phi_{B}$ with a cohomological hamiltonian vector field $Q_{B}=\left\{S_{B}, \bullet\right\}$, where $S_{B}$ is a certain odd function on $\Phi_{B}$ satisfying the Maurer-Cartan equation $\left\{S_{B}, S_{B}\right\}=0$. A natural candidate for the coboundary operator $\delta_{B}: \mathcal{H}_{B} \rightarrow \mathcal{H}_{B}$ is the quantization of the Maurer-Cartan element $S_{B}$.

In the case of the one-dimensional Chern-Simons theory, we have the target space $\mathcal{M}=\Pi \mathfrak{g}$, the phase space associated to a point $\Phi_{p t}=\Pi \mathfrak{g}$, the Maurer-Cartan element (4) $S_{p t}=\frac{1}{6} f^{a b c} X^{a} X^{b} X^{c}$, the space of states for a point (80) $\mathcal{H}_{p t}=\operatorname{Fun}(\Pi \mathfrak{h})-$ the geometric quantization space for $\Phi_{p t}=\Pi \mathfrak{g}$ (cf. Remark 22). The coboundary (up to possible anomaly (83)) operator for a point $\delta_{p t}$ is the quantization of $S_{p t}$, see (81). For other AKSZ theories, spaces of states constructed in this way will typically be infinite-dimensional complexes. However one may hope to pass to finite-dimensional deformation retracts of $\left(\mathcal{H}_{B}, \delta_{B}\right)$ by means of the homological perturbation theory.

1.4. Authorship. The idea of looking at the one-dimensional Chern-Simons theory and some parts of Sect. 3 (operator formalism approach) are joint work of both authors (the idea to use operator formalism for the one-dimensional Chern-Simons theory was suggested by A. A.). Other parts of the paper are due to P. M.

\section{Simplicial Chern-Simons Theory on the Circle}

In this section we study the Chern-Simons theory on the circle in Batalin-Vilkovisky (BV) formalism and construct an effective BV action induced on cochains of a triangulation. 
Much of this discussion is inspired by [7] and [15]. In particular, the reader is referred to Sects. 2 and 3.2 of [7] for details of the effective BV action construction.

2.1. Continuum theory on the circle: fields, BV structure, action. Let $\mathfrak{g}$ be a quadratic Lie algebra with Lie bracket [, ] and non-degenerate ad-invariant pairing (, ). We will denote by $\left\{T^{a}\right\}$ an orthonormal basis in $\mathfrak{g}$ and by $f^{a b c}=\left(T^{a},\left[T^{b}, T^{c}\right]\right)$ the structure constants in this basis. We will also use the Einstein summation convention for the Lie algebra indices.

The Chern-Simons theory on a 3-manifold $M$ can be constructed as an AKSZ sigma model [1] with the space of fields

$$
\mathcal{F}=\operatorname{Maps}(\Pi T M, \Pi \mathfrak{g})=\Pi \mathfrak{g} \otimes \Omega^{\bullet}(M) .
$$

That is, $\mathcal{F}$ is the space of maps of super-manifolds from the parity-shifted tangent bundle of $M$ to the parity-shifted Lie algebra. Equivalently, this is the space of differential forms on $M$ with values in $\Pi \mathfrak{g}$. From the canonical integration measure on $\Pi T M$ and the even symplectic structure $\omega_{\Pi \mathfrak{g}}=\frac{1}{2} \delta X^{a} \wedge \delta X^{a}$ on $\Pi \mathfrak{g}$ (we denote by $\left\{X^{a}\right\}$ the set of odd coordinates on $\Pi \mathfrak{g}$ associated to the orthonormal basis $\left\{T^{a}\right\}$ on $\mathfrak{g}$ ) one constructs an odd symplectic form (the "BV 2-form") on $\mathcal{F}$ :

$$
\omega=\frac{1}{2} \int_{M}(\delta \alpha, \delta \alpha)
$$

Here the superfield $\alpha$ is the canonical odd map (the parity-shifted identity operator)

$$
\alpha: \mathcal{F} \rightarrow \mathfrak{g} \otimes \Omega^{\bullet}(M)
$$

which can be viewed as the generating function for coordinates on $\mathcal{F}$ with values in $\mathfrak{g}$-valued differential forms on $M$. By splitting $\alpha$ into components according to the degrees of differential forms we obtain

$$
\alpha=A^{(0)}+A^{(1)}+A^{(2)}+A^{(3)},
$$

where $A^{(p)}$ takes values in $\mathfrak{g}$-valued $p$-forms. ${ }^{1}$ Since $\alpha$ is totally odd, $A^{(0)}$ and $A^{(2)}$ are intrinsically odd, $A^{(1)}$ and $A^{(3)}$ are intrinsically even (the intrinsic parity is the total parity minus the de Rham degree modulo 2). The Chern-Simons action is built of the 1 -form $\frac{1}{2} X^{a} \wedge \delta X^{a}$ on $\Pi \mathfrak{g}$ (which is a primitive for $\omega_{\Pi \mathfrak{g}}$ ) and the odd function on $\Pi \mathfrak{g}$

$$
\theta=\frac{1}{6} f^{a b c} X^{a} X^{b} X^{c}
$$

which satisfies $\{\theta, \theta\}_{\Pi \mathfrak{g}}=0$. The action is given by formula,

$$
S=\int_{M} \frac{1}{2}(\alpha, d \alpha)+\frac{1}{6}(\alpha,[\alpha, \alpha])
$$

By the general construction [1], $S$ satisfies the classical master equation

$$
\{S, S\}=0,
$$

where $\{$,$\} is the BV anti-bracket on functions on \mathcal{F}$ defined by the odd symplectic form $\omega$.

\footnotetext{
1 In the BV formalism, $A^{(1)}$ is the "classical field", $A^{(0)}$ is the "ghost"; $A^{(2)}$ and $A^{(3)}$ are the "anti-fields" for $A^{(1)}$ and $A^{(0)}$, respectively.
} 
We would like to define the one-dimensional Chern-Simons theory on the circle $\mathcal{S}^{1}$ by substituting $M=\mathcal{S}^{1}$ into the construction described above. Then, the space of fields becomes

$$
\mathcal{F}=\operatorname{Maps}\left(\Pi T \mathcal{S}^{1}, \Pi \mathfrak{g}\right)=\Pi \mathfrak{g} \otimes \Omega^{0}\left(\mathcal{S}^{1}\right) \oplus \Pi \mathfrak{g} \otimes \Omega^{1}\left(\mathcal{S}^{1}\right) .
$$

The superfield $\alpha$ can now be written as

$$
\alpha=\psi+A,
$$

where the component $\psi=T^{a} \psi^{a}(\tau)$ takes values in $\mathfrak{g}$-valued functions on the circle and is intrinsically odd ( $\tau$ is the coordinate on $\mathcal{S}^{1}$ ); and the component $A=d \tau T^{a} A^{a}(\tau)$ takes values in $\mathfrak{g}$-valued 1 -forms on the circle and is intrinsically even. Thus, $\left\{\psi^{a}(\tau), A^{a}(\tau)\right\}$ are odd and even coordinates on $\mathcal{F}$, respectively. The space $\mathcal{F}$ is equipped with an odd symplectic structure (2):

$$
\omega=\int_{\mathcal{S}^{1}}(\delta \psi, \delta A)
$$

defining the anti-bracket $\{\bullet, \bullet\}: \operatorname{Fun}(\mathcal{F}) \times \operatorname{Fun}(\mathcal{F}) \rightarrow \operatorname{Fun}(\mathcal{F})$,

$$
\{f, g\}=\int_{\mathcal{S}^{1}} d \tau f\left(\frac{\overleftarrow{\delta}}{\delta \psi^{a}(\tau)} \frac{\vec{\delta}}{\delta A^{a}(\tau)}-\frac{\overleftarrow{\delta}}{\delta A^{a}(\tau)} \frac{\vec{\delta}}{\delta \psi^{a}(\tau)}\right) g
$$

and the BV Laplacian $\Delta: \operatorname{Fun}(\mathcal{F}) \rightarrow \operatorname{Fun}(\mathcal{F})$

$$
\Delta f=\int_{\mathcal{S}^{1}} d \tau \frac{\delta}{\delta \psi^{a}(\tau)} \frac{\delta}{\delta A^{a}(\tau)} f .
$$

Note that the operator $\Delta$ is ill-defined on local functionals.

The action (5) can be written in terms of components (6) of the superfield as

$$
S=\frac{1}{2} \int_{\mathcal{S}^{1}}((\psi, d \psi)+(\psi,[A, \psi])) .
$$

Here $d$ is the de Rham differential on $\mathcal{S}^{1}$. By the general AKSZ construction, ${ }^{2}$ the action $S$ satisfies the classical master equation

$$
\{S, S\}=0 .
$$

Naiively, one could also say that the unimodularity of $\mathfrak{g}$ implies unimodularity of $\mathfrak{g} \otimes \Omega^{\bullet}\left(\mathcal{S}^{1}\right)$, and therefore the quantum master equation is fulfilled

$$
\frac{1}{2}\{S, S\}+\hbar \Delta S=0
$$

However, $\Delta S$ is ill-defined in continuum theory.

Remark 1. The $\mathbb{Z}_{2}$-grading on the space of fields of the Chern-Simons theory on a 3 -manifold can be promoted to a $\mathbb{Z}$-grading (by setting $\mathcal{F}=\operatorname{Maps}(T[1] M, \mathfrak{g}[1]$ )) in such a way that the odd symplectic form $\Omega$ attains grade ${ }^{3}-1$ (so that the anti-bracket has degree +1 ) and the action $S$ is in degree zero. However, this does not apply to the onedimensional Chern-Simons theory which is essentially $\mathbb{Z}_{2}$-graded: there is no consistent $\mathbb{Z}$-grading on the space of fields.

\footnotetext{
2 Or, in the algebraic language, due to relations (Leibniz identity, Jacobi identity, cyclicity of differential, cyclicity of Lie bracket) in the cyclic dg Lie algebra $\mathfrak{g} \otimes \Omega^{\bullet}\left(\mathcal{S}^{1}\right)$, cf. [7].

${ }^{3}$ Grade is defined as the total degree minus the de Rham degree of a differential form.
} 
2.2. Effective action on the cohomology of the circle.

2.2.1. Harmonic gauge. Let's split the space of differential forms on the circle into constant 0 - and 1 -forms and those with vanishing integral ${ }^{4}: \Omega^{\bullet}\left(\mathcal{S}^{1}\right)=\Omega^{\prime \bullet}\left(\mathcal{S}^{1}\right) \oplus \Omega^{\prime \prime \bullet}\left(\mathcal{S}^{1}\right)$, where

$$
\begin{gathered}
\Omega^{\prime \bullet}\left(\mathcal{S}^{1}\right)=\{f+d \tau g \mid f, g \in \mathbb{R}\} \\
\Omega^{\prime \prime \bullet}\left(\mathcal{S}^{1}\right)=\left\{f^{\prime \prime}(\tau)+d \tau g^{\prime \prime}(\tau) \mid \int_{\mathcal{S}^{1}} d \tau f^{\prime \prime}(\tau)=0, \int_{\mathcal{S}^{1}} d \tau g^{\prime \prime}(\tau)=0\right\} .
\end{gathered}
$$

It induces the splitting for fields into infrared and ultraviolet parts $\mathcal{F}=\mathcal{F}^{\prime} \oplus \mathcal{F}^{\prime \prime}$, where

$$
\begin{aligned}
\mathcal{F}^{\prime} & =\left\{\psi_{0}+d \tau A_{0} \mid \psi_{0} \in \Pi \mathfrak{g}, A_{0} \in \mathfrak{g}\right\} \\
\mathcal{F}^{\prime \prime} & =\left\{\psi^{\prime \prime}+A^{\prime \prime} \mid \int_{\mathcal{S}^{1}} d \tau \psi^{\prime \prime}(\tau)=0, \int_{\mathcal{S}^{1}} A^{\prime \prime}=0\right\}
\end{aligned}
$$

This splitting respects both the BV 2-form and the de Rham differential. We define the Lagrangian subspace $\mathcal{L} \subset \mathcal{F}^{\prime \prime}$ as

$$
\mathcal{L}=\left\{\psi^{\prime \prime}+A^{\prime \prime} \mid A^{\prime \prime}=0\right\}
$$

2.2.2. Effective action on cohomology. ${ }^{5}$ We define the effective action $W$ on $\mathcal{F}^{\prime}$ by the fiber BV integral

$$
\begin{aligned}
e^{\frac{1}{\hbar} W\left(\psi_{0}, A_{0}, \hbar\right)} & \left.=\int_{\mathcal{L}} e^{\frac{1}{\hbar} S\left(\psi_{0}+\psi^{\prime \prime}\right.}, d \tau A_{0}+A^{\prime \prime}\right) \\
& =\int \mathcal{D} \psi^{\prime \prime} e^{\frac{1}{2 \hbar} \int_{\mathcal{S}^{1}}\left(\psi_{0}+\psi^{\prime \prime},\left(d+d \tau \operatorname{ad}_{A_{0}}\right)\left(\psi_{0}+\psi^{\prime \prime}\right)\right)} .
\end{aligned}
$$

The Lagrangian subspace $\mathcal{L} \subset \mathcal{F}^{\prime \prime}$ is uniquely ${ }^{6}$ fixed by the requirement that the "free" part of the action $\frac{1}{2} \int_{\mathcal{S}^{1}}(\psi, d \psi)$ be non-degenerate when restricted to $\mathcal{L}$.

Integral (12) is Gaussian, and it yields the following result.

Proposition 1. The effective BV action $W$ of the one-dimensional Chern-Simons theory is given by

$$
e^{\frac{1}{\hbar} W\left(\psi_{0}, A_{0}, \hbar\right)}=\operatorname{det}_{\mathfrak{g}}^{1 / 2}\left(\frac{\sinh \frac{\operatorname{ad}_{A_{0}}}{2}}{\frac{\operatorname{ad}_{A_{0}}}{2}}\right) \cdot e^{-\frac{1}{2 \hbar}\left(\psi_{0}, \operatorname{ad}_{A_{0}} \psi_{0}\right)}
$$

\footnotetext{
${ }^{4}$ Properties of being constant for a 1 -form and being of integral zero for a 0 -form are non-covariant. This is not a problem as choosing a gauge always relies on introducing some additional structure. In the case of harmonic gauge, this extra structure is the parametrization of the circle.

${ }^{5}$ More precisely, we consider the effective action as a function on $\Pi \mathfrak{g} \otimes H^{\bullet}\left(\mathcal{S}^{1}\right)$, i.e. on the parity-shifted de Rham cohomology of the circle (which we represented by harmonic forms) with coefficients in $\mathfrak{g}$.

6 This a special property of the one-dimensional theory related to the fact that the de Rham operator $d: \Omega^{\prime \prime 0}\left(\mathcal{S}^{1}\right) \rightarrow \Omega^{\prime \prime 1}\left(\mathcal{S}^{1}\right)$ is an isomorphism, and there is unique chain homotopy $K=d^{-1}: \Omega^{\prime \prime 1}\left(\mathcal{S}^{1}\right) \rightarrow$ $\Omega^{\prime \prime 0}\left(\mathcal{S}^{1}\right)$.
} 
A simple form of the 0-loop part is due to the fact that multiplication by constant 1 -forms respects the splitting of forms into infrared and ultraviolet parts $(9,10){ }^{7}$ The functional determinant is easily computed e.g. by using the exponential basis $\left\{e^{2 \pi i k \tau}\right\}$ for 0 -forms on the circle.

The effective action (13) satisfies the quantum master equation

$$
\frac{\partial}{\partial \psi_{0}^{a}} \frac{\partial}{\partial A_{0}^{a}} e^{\frac{1}{\hbar} W\left(\psi_{0}, A_{0}, \hbar\right)}=0 .
$$

The classical master equation is implied by the Jacobi identity and by cyclicity property of the Lie bracket on $\mathfrak{g}$; the quantum part of master equation follows from the fact that the one-loop part of $W$ is manifestly ad-invariant.

2.3. Simplicial Chern-Simons action on circle. Let's assume that the circle $\mathcal{S}^{1}$ is glued of $n$ intervals $\mathcal{I}_{1}=\left[\mathfrak{p}_{1}, \mathfrak{p}_{2}\right], \ldots, \mathcal{I}_{n}=\left[\mathfrak{p}_{n}, \mathfrak{p}_{1}\right]$ (where $\mathfrak{p}_{1}, \ldots, \mathfrak{p}_{n}$ is a cyclically ordered collection of points on the circle), and that each interval $\mathcal{I}_{k}$ is equipped with a coordinate function $\tau: \mathcal{I}_{k} \rightarrow[0,1]$. We will denote a point of $\mathcal{I}_{k}$ with coordinate $\tau$ by $(k, \tau)$. We will assume $n$ to be odd (otherwise, our gauge will be inconsistent, see Remark 2). We denote this "triangulation" of the circle by $\Xi_{n}$.

2.3.1. Cyclic Whitney gauge. Splitting of fields into infrared and ultraviolet parts is defined by splitting for differential forms on the circle $\Omega^{\bullet}\left(\mathcal{S}^{1}\right)=\Omega_{\Xi_{n}}^{\prime \bullet}\left(\mathcal{S}^{1}\right) \oplus \Omega_{\Xi_{n}}^{\prime \prime \bullet}\left(\mathcal{S}^{1}\right)$, where we split 0 -forms into continuous piecewise-linear ones and those with vanishing integrals over each $\mathcal{I}_{k}$, and we split 1-forms into piecewise-constant ones and the orthogonal complement of piecewise-linear 0 -forms:

$$
\begin{aligned}
& \Omega_{\Xi_{n}}^{\prime \bullet}\left(\mathcal{S}^{1}\right)=\left\{f^{\prime}+d \tau g^{\prime} \quad\left|\quad f^{\prime}\right|_{\mathcal{I}_{k}}=(1-\tau) f_{k}+\tau f_{k+1},\left.g^{\prime}\right|_{\mathcal{I}_{k}}=g_{k} \forall k\right\}, \\
& \Omega_{\Xi_{n}}^{\prime \prime \bullet}\left(\mathcal{S}^{1}\right)=\left\{f^{\prime \prime}+d \tau g^{\prime \prime} \quad \mid \quad \int_{\mathcal{I}_{k}} d \tau f^{\prime \prime}=0, \int_{I_{k}} \tau d \tau g^{\prime \prime}+\int_{I_{k+1}}(1-\tau) d \tau g^{\prime \prime}=0 \forall k\right\},
\end{aligned}
$$

where $f_{k}, g_{k} \in \mathbb{R}$ are numbers. Thus, $\Omega_{\Xi_{n}}^{\prime \bullet}\left(\mathcal{S}^{1}\right) \cong \mathbb{R}^{n \mid n}$ (as a super-space). As a cochain complex, $\Omega_{\Xi_{n}}^{\bullet}\left(\mathcal{S}^{1}\right)$ is isomorphic to $C^{\bullet}\left(\Xi_{n}\right)$, the cochain complex of the simplicial complex $\Xi_{n}$. As in Sect. 2.2.1, this splitting agrees with the de Rham differential, and the associated splitting for fields $\mathcal{F}=\mathcal{F}^{\prime} \oplus \mathcal{F}^{\prime \prime}$ agrees with the BV 2-form.

We call splitting (14) the "cyclic Whitney gauge", because our representatives $\Omega^{\prime \bullet}$ for cell cochains of triangulation are exactly the Whitney forms [17] for $\Xi_{n}$. The word "cyclic" indicates that $\Omega^{\prime \prime}$ is constructed as an orthogonal complement of $\Omega^{\prime \bullet}$ with respect to the Poincaré pairing $\int_{\mathcal{S}^{1}} \bullet \wedge \bullet$.

Coordinates on $\mathcal{F}^{\prime}$ are given by values of $\psi^{\prime}$ at the vertices of triangulation:

$$
\psi_{k}=\psi^{\prime}\left(\mathfrak{p}_{k}\right) \in \Pi \mathfrak{g},
$$

and by integrals of $A$ over intervals:

$$
A_{k}=\int_{\mathcal{I}_{k}} A^{\prime} \in \mathfrak{g} .
$$

\footnotetext{
7 Higher order terms in the 0-loop effective action would correspond to Massey operations on the de Rham cohomology (cf. $[7,15])$. In the case of the circle, Massey operations vanish.
} 
The BV 2-form on $\mathcal{F}^{\prime}$ is given by

$$
\omega^{\prime}=\sum_{k=1}^{n} \frac{\delta \psi_{k}^{a}+\delta \psi_{k+1}^{a}}{2} \wedge \delta A_{k}^{a}
$$

We will denote $\mathcal{F}^{\prime}=\mathcal{F}_{\Xi_{n}}$ to emphasize its dependence on $n$. We have $\mathcal{F}_{\Xi_{n}} \cong \Pi \mathfrak{g} \otimes$ $C^{\bullet}\left(\Xi_{n}\right)$.

Remark 2. The requirement that $n$ be odd is needed since for $n$ even the piecewise-linear 0-form

$$
f(k, \tau)=(-1)^{k}(\tau-1 / 2)
$$

belongs to both the infrared and ultraviolet subspaces.

As in Sect. 2.2.1, we define the Lagrangian subspace $\mathcal{L} \subset \mathcal{F}^{\prime \prime}$ by setting the 1-form part of the ultraviolet field to zero (11).

2.3.2. Chain homotopy, dressed chain homotopy. Let us define the infrared projector $\mathcal{P}^{\prime}: \Omega^{\bullet}\left(\mathcal{S}^{1}\right) \rightarrow \Omega_{\Xi_{n}}^{\prime \bullet}\left(\mathcal{S}^{1}\right)$ by formula

$$
\begin{aligned}
f+d \tau \cdot g \mapsto & \sum_{k=1}^{n}\left(\int_{\mathcal{I}_{k}} d \tau^{\prime} f-(1-2 \tau) \cdot\left(\int_{\mathcal{I}_{k+1}} d \tau^{\prime} f-\int_{\mathcal{I}_{k+2}} d \tau^{\prime} f\right.\right. \\
& \left.\left.+\cdots+\int_{\mathcal{I}_{k-2}} d \tau^{\prime} f-\int_{\mathcal{I}_{k-1}} d \tau^{\prime} f\right)\right) \theta_{\mathcal{I}_{k}} \\
& +d \tau \sum_{k=1}^{n}\left(\int_{\mathcal{I}_{k}} d \tau^{\prime} g+\int_{\mathcal{I}_{k+1}}\left(1-2 \tau^{\prime}\right) d \tau^{\prime} g-\int_{\mathcal{I}_{k+2}}\left(1-2 \tau^{\prime}\right) d \tau^{\prime} g\right. \\
& \left.+\cdots-\int_{\mathcal{I}_{k-1}}\left(1-2 \tau^{\prime}\right) d \tau^{\prime} g\right) \theta_{\mathcal{I}_{k}}
\end{aligned}
$$

where $\theta_{\mathcal{I}_{k}}$ is the function on the circle with value 1 on $\mathcal{I}_{k}$ and zero elsewhere.

The chain homotopy $\kappa: \Omega^{1}\left(\mathcal{S}^{1}\right) \rightarrow \Omega^{0}\left(\mathcal{S}^{1}\right)$ is uniquely defined by the properties

$$
\begin{aligned}
d \kappa+\kappa d & =\mathrm{id}-\mathcal{P}^{\prime}, \\
\mathcal{P}^{\prime} \kappa & =0, \\
\kappa \mathcal{P}^{\prime} & =0 .
\end{aligned}
$$

Lemma 1. The operator $\kappa$ defined by relations (19), (20), (21) acts on the 1-form $d \tau \cdot g \in \Omega^{1}\left(\mathcal{S}^{1}\right) b y^{8}$

$$
\kappa(d \tau \cdot g)(k, \tau)=\sum_{k^{\prime}=1}^{n} \int_{\mathcal{I}_{k^{\prime}}} d \tau^{\prime} \kappa\left((k, \tau),\left(k^{\prime}, \tau^{\prime}\right)\right) g\left(k^{\prime}, \tau^{\prime}\right),
$$

\footnotetext{
${ }^{8}$ Recall that $(k, \tau)$ denotes a point on the circle which belongs to the interval $\mathcal{I}_{k}$ and which has a local coordinate $\tau$. Hence, the integral kernel here is actually a function on $\mathcal{S}^{1} \times \mathcal{S}^{1}$.
} 
where the integral kernel is given by

$$
\kappa\left((k, \tau),\left(k^{\prime}, \tau^{\prime}\right)\right)= \begin{cases}\theta\left(\tau-\tau^{\prime}\right)-\frac{1}{2}-\tau+\tau^{\prime} & \text { if } k=k^{\prime} \\ (-1)^{k-k^{\prime}} 2\left(\frac{1}{2}-\tau\right)\left(\frac{1}{2}-\tau^{\prime}\right) & \text { if } k^{\prime}<k<k^{\prime}+n\end{cases}
$$

and $\theta$ is the unit step function. In addition, the kernel has the anti-symmetry property:

$$
\kappa\left(\left(k^{\prime}, \tau^{\prime}\right),(k, \tau)\right)=-\kappa\left((k, \tau),\left(k^{\prime}, \tau^{\prime}\right)\right) .
$$

To obtain formula (23), one observes that relations (19) and (20) imply the differential equation

$$
\frac{\partial}{\partial \tau} \kappa\left((k, \tau),\left(k^{\prime}, \tau^{\prime}\right)\right)=\delta_{k, k^{\prime}} \delta\left(\tau-\tau^{\prime}\right)+C_{k}\left(k^{\prime}, \tau^{\prime}\right)
$$

subject to conditions

$$
\int_{0}^{1} d \tau \kappa\left((k, \tau),\left(k^{\prime}, \tau^{\prime}\right)\right)=0, \quad \kappa\left((k, 1),\left(k^{\prime}, \tau^{\prime}\right)\right)=\kappa\left((k+1,0),\left(k^{\prime}, \tau^{\prime}\right)\right) \quad \forall k .
$$

Here $C_{k}\left(k^{\prime}, \tau^{\prime}\right)$ are some functions independent of $\tau$. Solving (24) together with (25) immediately yields (23). This proves the uniqueness property. In order to prove existence, one checks that (23) satisfies (19), (20), (21).

We will also need the "dressed" chain homotopy (i.e. dressed by the connection) $\kappa_{A^{\prime}}: \mathfrak{g} \otimes \Omega^{1}\left(\mathcal{S}^{1}\right) \rightarrow \mathfrak{g} \otimes \Omega^{0}\left(\mathcal{S}^{1}\right)$, where $A^{\prime}=\sum_{k=1}^{n} A_{k} \theta_{\mathcal{I}_{k}}$ is a piecewise-constant $\mathfrak{g}$-valued 1 -form on the circle. The operator $\kappa_{A^{\prime}}$ is uniquely defined by the properties

$$
\begin{aligned}
\left(\mathrm{id}-\mathcal{P}^{\prime}\right) d_{A^{\prime}} \kappa_{A^{\prime}}+\kappa_{A^{\prime}} d_{A^{\prime}}\left(\mathrm{id}-\mathcal{P}^{\prime}\right) & =\mathrm{id}-\mathcal{P}^{\prime}, \\
\mathcal{P}^{\prime} \kappa_{A^{\prime}} & =0, \\
\kappa_{A^{\prime}} \mathcal{P}^{\prime} & =0,
\end{aligned}
$$

where $d_{A^{\prime}}=d+\operatorname{ad}_{A^{\prime}}$. One can summarize these properties by saying that $\kappa_{A^{\prime}}$ : $\Omega_{\Xi_{n}}^{\prime \prime 1}\left(\mathcal{S}^{1}\right) \rightarrow \Omega_{\Xi_{n}}^{\prime \prime 0}\left(\mathcal{S}^{1}\right)$ is the inverse of the operator $\left(\mathrm{id}-\mathcal{P}^{\prime}\right) d_{A^{\prime}}\left(\mathrm{id}-\mathcal{P}^{\prime}\right): \Omega_{\Xi_{n}}^{\prime \prime 0}\left(\mathcal{S}^{1}\right) \rightarrow$ $\Omega_{\Xi_{n}}^{\prime \prime 1}\left(\mathcal{S}^{1}\right)$. The dressed chain homotopy can either be expressed perturbatively as a series

$$
\kappa_{A^{\prime}}=\kappa-\kappa \operatorname{ad}_{A^{\prime}} \kappa+\kappa \operatorname{ad}_{A^{\prime}} \kappa \operatorname{ad}_{A^{\prime}} \kappa-\cdots,
$$

or it can be computed explicitly by solving the differential equation (26).

To present an explicit formula for the integral kernel of $\kappa_{A^{\prime}}$ (defined as in (22)), we have to first introduce some notation. Let $F(\mathcal{A}, \tau)$ be an $\operatorname{End}(\mathfrak{g})$-valued function on the interval $[0,1]$ depending on an anti-symmetric matrix $\mathcal{A} \in \mathfrak{s o}(\mathfrak{g}) \subset$ End(g) and defined by the following properties:

$$
\begin{aligned}
F(\mathcal{A}, \bullet) & \in \operatorname{Span}_{\operatorname{End}(\mathfrak{g})}\left(1, e^{-\mathcal{A} \tau}\right), \\
\int_{0}^{1} d \tau F(\mathcal{A}, \tau) & =0 \\
F(\mathcal{A}, 0) & =1 .
\end{aligned}
$$


Property (29) is equivalently stated as $(d+\mathcal{A}) F(\mathcal{A}, \bullet)=$ const (this constant depends on $\mathcal{A}$ ). It is convenient to introduce notation

$$
R(\mathcal{A})=-F(\mathcal{A}, 1) \in \operatorname{End}(\mathfrak{g}) .
$$

More explicitly, we have

$$
\begin{aligned}
F(\mathcal{A}, \tau) & =\frac{\frac{1}{2}\left(\operatorname{coth} \frac{\mathcal{A}}{2}+1\right) e^{-\mathcal{A} \tau}-\mathcal{A}^{-1}}{\frac{1}{2}\left(\operatorname{coth} \frac{\mathcal{A}}{2}+1\right)-\mathcal{A}^{-1}}, \\
R(\mathcal{A}) & =-\frac{\mathcal{A}^{-1}+\frac{1}{2}-\frac{1}{2} \operatorname{coth} \frac{\mathcal{A}}{2}}{\mathcal{A}^{-1}-\frac{1}{2}-\frac{1}{2} \operatorname{coth} \frac{\mathcal{A}}{2}}
\end{aligned}
$$

Since the function $x^{-1}-\frac{1}{2} \operatorname{coth} \frac{x}{2}$ is analytic at $x=0$, expressions (30), (31) are in fact well-defined for non-invertible $\mathcal{A}$. We will use notation

$$
\mu_{k}\left(A^{\prime}\right)=R\left(\operatorname{ad}_{A_{k-1}}\right) R\left(\operatorname{ad}_{A_{k-2}}\right) \cdots R\left(\operatorname{ad}_{A_{k+1}}\right) R\left(\operatorname{ad}_{A_{k}}\right) \in \operatorname{End}(\mathfrak{g}) .
$$

The following reflection properties

$$
F(-\mathcal{A}, \tau)=-\frac{F(\mathcal{A}, 1-\tau)}{R(\mathcal{A})}, \quad R(-\mathcal{A})=R(\mathcal{A})^{-1}, \quad \mu_{k}\left(A^{\prime}\right)^{T}=\mu_{k}\left(A^{\prime}\right)^{-1}
$$

mean that $R(\mathcal{A})$ and $\mu_{k}\left(A^{\prime}\right)$ take values in orthogonal matrices $O(\mathfrak{g}) \subset \operatorname{End}(\mathfrak{g})$.

We can now present the result for the integral kernel of the dressed chain homotopy $\kappa_{A^{\prime}}$ :

Lemma 2. The operator $\kappa_{A^{\prime}}$ defined by relations (26), (27), (28) acts on a $\mathfrak{g}$-valued 1-form $d \tau \cdot \beta \in \mathfrak{g} \otimes \Omega^{1}\left(\mathcal{S}^{1}\right)$ by formula

$$
\kappa_{A^{\prime}}(d \tau \cdot \beta)(k, \tau)=\sum_{k^{\prime}=1}^{n} \int_{\mathcal{I}_{k^{\prime}}} d \tau^{\prime} \cdot \kappa_{A^{\prime}}\left((k, \tau),\left(k^{\prime}, \tau^{\prime}\right)\right) \circ \beta\left(k^{\prime}, \tau^{\prime}\right),
$$

where the integral kernel is given by

$$
\begin{aligned}
& \kappa_{A^{\prime}}\left((k, \tau),\left(k^{\prime}, \tau^{\prime}\right)\right) \\
& = \begin{cases}\left(\theta\left(\tau-\tau^{\prime}\right)-\frac{1}{2}+\frac{1}{2} \operatorname{coth} \frac{\operatorname{ad}_{A_{k}}}{2}\right) e^{\left(\tau^{\prime}-\tau\right) \operatorname{ad}_{A_{k}}-\left(\operatorname{ad}_{A_{k}}\right)^{-1}} & \text { if } k=k^{\prime}, \\
& +F\left(\operatorname{ad}_{A_{k}}, \tau\right)\left(\frac{1}{1+\mu_{k}\left(A^{\prime}\right)}-\frac{1}{1+R\left(\operatorname{ad}_{A_{k}}\right)}\right) F\left(-\operatorname{ad}_{A_{k}}, \tau^{\prime}\right) \\
(-1)^{k-k^{\prime}} F\left(\operatorname{ad}_{A_{k}}, \tau\right) R\left(\operatorname{ad}_{A_{k-1}}\right) \cdots R\left(\operatorname{ad}_{A_{k^{\prime}}}\right) \frac{1}{1+\mu_{k^{\prime}}\left(A^{\prime}\right)} F\left(-\operatorname{ad}_{A_{k^{\prime}}}, \tau^{\prime}\right) & \text { if } k^{\prime}<k<k^{\prime}+n .\end{cases}
\end{aligned}
$$

This integral kernel is anti-symmetric:

$$
\kappa_{A^{\prime}}\left(\left(k^{\prime}, \tau^{\prime}\right),(k, \tau)\right)^{T}=-\kappa_{A^{\prime}}\left((k, \tau),\left(k^{\prime}, \tau^{\prime}\right)\right) .
$$

To obtain (34) we proceed as in the derivation of (23). The differential equation implied by (26) is as follows:

$$
\left(\frac{\partial}{\partial \tau}+\operatorname{ad}_{A_{k}}\right) \kappa_{A^{\prime}}\left((k, \tau),\left(k^{\prime}, \tau^{\prime}\right)\right)=\delta_{k, k^{\prime}} \delta\left(\tau-\tau^{\prime}\right)+C_{k}\left(k^{\prime}, \tau^{\prime}\right) .
$$

Conditions (25) are still fulfilled. Solving (35) with these conditions imposed yields formula (34). 
2.3.3. Simplicial action. Simplicial Chern-Simons action $S_{\Xi_{n}}$ for the triangulation $\Xi_{n}$ of circle is defined by the fiber BV integral

$$
e^{\frac{1}{\hbar} S_{\Xi_{n}}\left(\psi^{\prime}, A^{\prime}, \hbar\right)}=\int_{\mathcal{L}} e^{\frac{1}{\hbar} S\left(\psi^{\prime}+\psi^{\prime \prime}, A^{\prime}+A^{\prime \prime}\right)}
$$

As in the case of induction to cohomology, one puts $\left.A^{\prime \prime}\right|_{\mathcal{L}}=0$, and the integral becomes Gaussian:

$$
e^{\frac{1}{\hbar} S_{\Xi_{n}}\left(\psi^{\prime}, A^{\prime}, \hbar\right)}=\int \mathcal{D} \psi^{\prime \prime} e^{\frac{1}{2 \hbar} \int_{\mathcal{S}^{1}}\left(\psi^{\prime}+\psi^{\prime \prime}, d_{A^{\prime}}\left(\psi^{\prime}+\psi^{\prime \prime}\right)\right)} .
$$

Expanding the integrand as

$$
\left(\psi^{\prime}, d_{A^{\prime}} \psi^{\prime}\right)+\left(\psi^{\prime \prime}, d \psi^{\prime \prime}\right)+\left(\psi^{\prime \prime}, \operatorname{ad}_{A^{\prime}} \psi^{\prime \prime}\right)+2\left(\psi^{\prime \prime}, \operatorname{ad}_{A^{\prime}} \psi^{\prime}\right),
$$

one can consider the second term as the "free" part of the action and the third and fourth terms as a perturbation. In this way, we arrive at the following Feynman diagram expansion for $S_{\Xi_{n}}$ :

$$
\begin{aligned}
S_{\Xi_{n}}= & \underbrace{\frac{1}{2}\left(\psi^{\prime}, d \psi^{\prime}\right)+\frac{1}{2}\left(\psi^{\prime}, \operatorname{ad}_{A^{\prime}} \psi^{\prime}\right)-\frac{1}{2}\left(\psi^{\prime}, \operatorname{ad}_{A^{\prime}} \kappa \operatorname{ad}_{A^{\prime}} \psi^{\prime}\right)+\frac{1}{2}\left(\psi^{\prime}, \operatorname{ad}_{A^{\prime}} \kappa \operatorname{ad}_{A^{\prime}} \kappa \operatorname{ad}_{A^{\prime}} \psi^{\prime}\right)-\cdots}_{S_{\Xi_{n}}^{0}} \\
& +\underbrace{\hbar \frac{1}{2 \cdot 2} \operatorname{tr}_{\mathfrak{g} \otimes \Omega^{0}\left(\mathcal{S}^{1}\right)}\left(\kappa \operatorname{ad}_{A^{\prime}} \kappa \operatorname{ad}_{A^{\prime}}\right)-\hbar \frac{1}{2 \cdot 3} \operatorname{tr}_{\mathfrak{g} \otimes \Omega^{0}\left(\mathcal{S}^{1}\right)}\left(\kappa \operatorname{ad}_{A^{\prime}} \kappa \operatorname{ad}_{A^{\prime}} \kappa \operatorname{ad}_{A^{\prime}}\right)+\cdots}_{\hbar S_{\Xi_{n}}^{1}}
\end{aligned}
$$

Here the first line is the sum of "tree" diagrams and the second line is the sum of "wheel diagrams".

The tree part of $S_{\Xi_{n}}$ can be expressed in terms of the dressed chain homotopy,

$$
S_{\Xi_{n}}^{0}=\frac{1}{2} \int_{\mathcal{S}^{1}}\left(\psi^{\prime}, d \psi^{\prime}\right)+\frac{1}{2} \int_{\mathcal{S}^{1}}\left(\psi^{\prime}, \operatorname{ad}_{A^{\prime}} \psi^{\prime}\right)-\frac{1}{2} \int_{\mathcal{S}^{1}}\left(\psi^{\prime}, \operatorname{ad}_{A^{\prime}} \kappa_{A^{\prime}} \operatorname{ad}_{A^{\prime}} \psi^{\prime}\right) .
$$

For the 1-loop part, we first write

$$
S_{\Xi_{n}}^{1}=\frac{1}{2} \operatorname{tr}_{\mathfrak{g} \otimes \Omega^{0}\left(\mathcal{S}^{1}\right)} \log \left(1+\kappa \operatorname{ad}_{A^{\prime}}\right) .
$$

Then, by using the general formula

$$
\frac{\partial}{\partial s} \operatorname{tr} \log M_{s}=\operatorname{tr}\left(M_{s}^{-1} \frac{\partial M_{s}}{\partial s}\right)
$$

one obtains

$$
\begin{aligned}
S_{\Xi_{n}}^{1} & =\int_{0}^{1} d s \frac{\partial}{\partial s}\left(\frac{1}{2} \operatorname{tr}_{\mathfrak{g} \otimes \Omega^{0}\left(\mathcal{S}^{1}\right)} \log \left(1+\kappa \operatorname{ad}_{s A^{\prime}}\right)\right) \\
& =\frac{1}{2} \int_{0}^{1} d s \operatorname{tr}_{\mathfrak{g} \otimes \Omega^{0}\left(\mathcal{S}^{1}\right)} \underbrace{\left(1+\kappa \operatorname{ad}_{s A^{\prime}}\right)^{-1} \kappa}_{\kappa_{s A^{\prime}}} \operatorname{ad}_{A^{\prime}} .
\end{aligned}
$$


One can evaluate the functional trace in the integrand in the coordinate representation (i.e. in the basis of delta-functions) by expressing it in terms of the integral kernel (34) restricted to the diagonal $\mathcal{S}^{1} \subset \mathcal{S}^{1} \times \mathcal{S}^{1}$ :

$$
S_{\Xi_{n}}^{1}=\int_{0}^{1} d s \frac{1}{2} \sum_{k=1}^{n} \int_{0}^{1} d \tau \operatorname{tr}_{\mathfrak{g}}\left(\kappa_{s A^{\prime}}((k, \tau),(k, \tau)) \operatorname{ad}_{A_{k}}\right) .
$$

There is an ambiguity in this expression since the integral kernel (34) is discontinuous on the diagonal. We regularize this ambiguity by using the convention

$$
\theta(0)=\frac{1}{2}
$$

Observe that the value assigned to $\theta(0)$ does not really matter: changing the convention for $\theta(0)$ changes $S_{\Xi_{\eta}}^{1}$ by $\propto \operatorname{tr}_{\mathfrak{g}} a_{A^{\prime}}=0$. It is interesting that the integral over the auxiliary parameter $s$ in (40) can be computed explicitly. By putting together (39) and (40) and substituting (34) we obtain the following explicit result for the simplicial Chern-Simons action on the triangulated circle.

Theorem 1. Simplicial Chern-Simons action on the triangulated circle is given by

$$
\begin{aligned}
S_{\Xi_{n}}= & -\frac{1}{2} \sum_{k=1}^{n}\left(\left(\psi_{k}, \psi_{k+1}\right)+\frac{1}{3}\left(\psi_{k}, \operatorname{ad}_{A_{k}} \psi_{k}\right)+\frac{1}{3}\left(\psi_{k+1}, \operatorname{ad}_{A_{k}} \psi_{k+1}\right)+\frac{1}{3}\left(\psi_{k}, \operatorname{ad}_{A_{k}} \psi_{k+1}\right)\right) \\
& +\frac{1}{2} \sum_{k=1}^{n}\left(\psi_{k+1}-\psi_{k},\left(\frac{1-R\left(\operatorname{ad}_{A_{k}}\right)}{2}\left(\frac{1}{1+\mu_{k}\left(A^{\prime}\right)}-\frac{1}{1+R\left(\operatorname{ad}_{A_{k}}\right)}\right) \frac{1-R\left(\operatorname{ad}_{A_{k}}\right)}{2 R\left(\operatorname{ad}_{A_{k}}\right)}\right.\right. \\
& \left.\left.+\left(\operatorname{ad}_{A_{k}}\right)^{-1}+\frac{1}{12} \operatorname{ad}_{A_{k}}-\frac{1}{2} \operatorname{coth} \frac{\operatorname{ad}_{A_{k}}}{2}\right) \circ\left(\psi_{k+1}-\psi_{k}\right)\right) \\
& +\frac{1}{2} \sum_{k^{\prime}=1}^{n} \sum_{k=k^{\prime}+1}^{k^{\prime}+n-1}(-1)^{k-k^{\prime}}\left(\psi_{k+1}-\psi_{k}, \frac{1-R\left(\operatorname{ad}_{A_{k}}\right)}{2} R\left(\operatorname{ad}_{A_{k-1}}\right) \cdots R\left(\operatorname{ad}_{A_{k^{\prime}}}\right)\right. \\
& \left.+\frac{1-R\left(\operatorname{ad}_{A_{k^{\prime}}}\right)}{1+\mu_{k^{\prime}}\left(\mathcal{A}^{\prime}\right)} \cdot \frac{1}{2 R\left(\operatorname{ad}_{A_{k^{\prime}}}\right)}\left(\psi_{k^{\prime}+1}-\psi_{k^{\prime}}\right)\right) \\
& +\hbar \frac{1}{2} \operatorname{tr}_{\mathfrak{g}} \log \left(\left(1+\mu_{\bullet}\left(A^{\prime}\right)\right) \prod_{k=1}^{n}\left(\frac{1}{1+R\left(\operatorname{ad}_{A_{k}}\right)} \cdot \frac{\sinh \frac{\operatorname{ad}_{A_{k}}}{2}}{\frac{\operatorname{ad}_{A_{k}}}{2}}\right)\right) .
\end{aligned}
$$

In the last term (the 1-loop contribution) $\mu_{\bullet}\left(A^{\prime}\right)$ stands for $\mu_{l}\left(A^{\prime}\right)$ for arbitrary $l$. For different $l$, these matrices differ by conjugation. Hence, the expression $\operatorname{det}_{\mathfrak{g}}\left(1+\mu_{\bullet}\left(A^{\prime}\right)\right)$ is well defined.

\subsubsection{Remarks.}

Remark 3. In deriving formula (42) we were sloppy about additive constants (we did not pay attention to normalization of the measure in the functional integral (37)). We chose an ad hoc normalization

$$
S_{\Xi_{n}}(0,0, \hbar)=-\hbar \frac{n-1}{2} \operatorname{dim} \mathfrak{g} \log 2
$$

which turns out to be consistent with the operator formalism (see Sect. 3.1). 
Remark 4. Setting $n=1$ in (42) yields the effective action on cohomology (13).

Remark 5. By expanding (42) in a power series with respect to $A^{\prime}$ we get back the perturbative expansion (38)),

$$
\begin{aligned}
S_{\Xi_{n}}= & -\frac{1}{2} \sum_{k=1}^{n}\left(\psi_{k}, \psi_{k+1}\right)-\frac{1}{2} \sum_{k=1}^{n}\left(\frac{1}{3}\left(\psi_{k}, \operatorname{ad}_{A_{k}} \psi_{k}\right)+\frac{1}{3}\left(\psi_{k+1}, \operatorname{ad}_{A_{k}} \psi_{k+1}\right)\right. \\
& \left.+\frac{1}{3}\left(\psi_{k}, \operatorname{ad}_{A_{k}} \psi_{k+1}\right)\right) \\
& +\frac{1}{2} \sum_{k^{\prime}=1}^{n} \sum_{k=k^{\prime}+1}^{k^{\prime}+n-1} \frac{1}{72}(-1)^{k-k^{\prime}}\left(\psi_{k+1}-\psi_{k}, \operatorname{ad}_{A_{k}} \operatorname{ad}_{A_{k^{\prime}}}\left(\psi_{k^{\prime}+1}-\psi_{k^{\prime}}\right)\right) \\
& -\hbar \frac{1}{2} \operatorname{dim} \mathfrak{g} \log 2+\hbar \frac{1}{2 \cdot 2}\left(\sum_{k=1}^{n} \frac{1}{12} \operatorname{tr}_{\mathfrak{g}}\left(\operatorname{ad}_{A_{k}}\right)^{2}\right. \\
& \left.+\sum_{k^{\prime}=1}^{n} \sum_{k=k^{\prime}+1}^{k^{\prime}+n-1} \frac{1}{36} \operatorname{tr}_{\mathfrak{g}}\left(\operatorname{ad}_{A_{k}} \operatorname{ad}_{A_{k^{\prime}}}\right)\right)+\mathcal{O}\left(\left(A^{\prime}\right)^{3}\right) .
\end{aligned}
$$

Remark 6. Naïvely, at large $n$ the simplicial action $S_{\Xi_{n}}$ can be viewed as a lattice approximation to the continuum action (8). For $\psi$ and $A$ fixed, we have

$$
S_{\Xi_{n}}\left(\left\{\psi_{k}=\psi(k / n)\right\}, \quad\left\{A_{k}=\int_{k / n}^{(k+1) / n} A\right\}\right) \longrightarrow S(\psi, A)+\mathcal{O}(1 / n)
$$

when $n$ tends to infinity. The point is that rather than being just an approximation the simplicial theory $\left(\mathcal{F}_{\Xi_{n}}, S_{\Xi_{n}}\right)$ is exactly equivalent to the continuum theory $(\mathcal{F}, S)$ for any finite $n$.

Remark 7. The simplicial theory is constructed by the fiber BV integral from the continuum theory. Hence, we expect the simplicial action to satisfy the quantum master equation

$$
\Delta_{\Xi_{n}} e^{\frac{1}{\hbar} S_{\Xi_{n}}}=0
$$

with BV Laplacian associated to the BV 2-form (17),

$$
\Delta_{\Xi_{n}}=\sum_{k=1}^{n} \frac{\partial}{\partial \tilde{\psi}_{k}^{a}} \frac{\partial}{\partial A_{k}^{a}},
$$

where

$$
\tilde{\psi}_{k}=\frac{\psi_{k}+\psi_{k+1}}{2}
$$

However, the BV Laplacian in the continuum theory is ill-defined. Therefore, the quantum master equation (44) is not automatic, and it must be checked independently. It is easy to do it in low degrees in $A^{\prime}$ by using the expansion (43). We will prove the quantum master equation via the operator approach in Sect. 3.2.3. 
Remark 8. Another property we expect from the simplicial theory is its compatibility with simplicial aggregations $\Xi_{n+2} \rightarrow \Xi_{n}$. We will prove this property in Sect. 3.2.2.

Remark 9. The gauge choice (14) is actually rigid up to diffeomorphisms of intervals $\mathcal{I}_{k}$. More exactly, if we require compatibility of the splitting $\Omega=\Omega^{\prime} \oplus \Omega^{\prime \prime}$ with the de Rham differential and with the pairing $\int_{\mathcal{S}^{1}} \bullet \wedge \bullet$ (i.e. so that $\Omega^{\prime}$ and $\Omega^{\prime \prime}$ are subcomplexes of $\Omega$, and $\Omega^{\prime \prime}$ is orthogonal to $\Omega^{\prime}$ ), then the splitting is completely determined by the images of basis 1-cochains of $\Xi_{n}$ in $\Omega^{1}\left(\mathcal{S}^{1}\right)$. If in addition, we require that the image of each basis 1-cochain $e_{k}^{(1)}$ be supported exactly on the respective interval $\mathcal{I}_{k} \subset \mathcal{S}^{1}$ (a kind of simplicial locality property), we obtain the splitting (14) up to diffeomorphisms of intervals $\mathcal{I}_{k}$ taking the representatives of basis 1 -cochains $e_{k}^{(1)}$ to constant forms $\theta_{\mathcal{I}_{k}} d \tau$. It is important to note that we are implicitly assuming that the splitting for fields is induced by the splitting for real-valued differential forms. If we drop this assumption and allow splittings of $\mathfrak{g} \otimes \Omega$ which are not obtained from a splitting of $\Omega$ by tensoring with $\mathfrak{g}$, we can introduce other gauges.

Remark 10. The embedding of cell cochains of $\Xi_{n}$ to the space of differential forms in (14) is the same as in the 1-dimensional simplicial BF theory [15]: 0-cochains are represented by continuous piecewise-linear functions, and 1-cochains are represented by piecewise-constant 1 -forms. However, the projection (18) is very different. In fact, it is non-local: for 0 -forms instead of evaluation at vertices (as in simplicial BF) we have a sum of integrals over all intervals $\mathcal{I}_{k}$ with certain signs. And for 1 -forms, instead of integration over one interval we have an integral over the whole circle with a certain piecewise-linear integral kernel. Thus, in the splitting $\Omega=\Omega^{\prime} \oplus \Omega^{\prime \prime}$ the infrared part is like in the simplicial BF theory, but the ultraviolet part is different.

Remark 11. The action (42) can be viewed as a generating function of a certain infinitystructure on $\mathfrak{g} \otimes C^{\bullet}\left(\Xi_{n}\right)$. In more detail, this is a structure of a loop-enhanced (or "quantum", or "unimodular") cyclic $L_{\infty}$ algebra with the structure maps (operations) $c_{k}^{(l)}: \wedge^{k}\left(\mathfrak{g} \otimes C^{\bullet}\left(\Xi_{n}\right)\right) \rightarrow \mathbb{R}$ related to the action (42) by

$$
S_{\Xi_{n}}=\sum_{l=0}^{1} \sum_{k=2}^{\infty} \frac{\hbar^{l}}{k !} c_{k}^{(l)}(\underbrace{\psi^{\prime}+A^{\prime}, \cdots, \psi^{\prime}+A^{\prime}}_{k}),
$$

where the superfield $\psi^{\prime}+A^{\prime}$ is understood as the parity-shifted identity map $\mathcal{F}_{\Xi_{n}} \rightarrow$ $\mathfrak{g} \otimes C^{\bullet}\left(\Xi_{n}\right)$ (as in (3)). The quantum master equation (44) generates a family of structure equations on operations $c_{k}^{(l)}$. In particular, $c_{k}^{(0)}$ satisfy the structure equations of the usual (nonunimodular) cyclic $L_{\infty}$ algebra. This algebraic structure on $\mathfrak{g}$-valued cochains of $\Xi_{n}$ can be viewed as a homotopy transfer of the unimodular cyclic DGLA structure on $\mathfrak{g} \otimes \Omega^{\bullet}\left(\mathcal{S}^{1}\right)$. Only the first two cyclic operations, $c_{2}^{(0)}: \wedge^{2}\left(\mathfrak{g} \otimes C^{\bullet}\left(\Xi_{n}\right)\right) \rightarrow \mathbb{R}$ and $c_{3}^{(0)}: \wedge^{3}\left(\mathfrak{g} \otimes C^{\bullet}\left(\Xi_{n}\right)\right) \rightarrow \mathbb{R}$ are simplicially-local. All the other operations are nonlocal. We can also use the pairing on $\mathfrak{g} \otimes C^{\bullet}\left(\Xi_{n}\right)$ (induced by the pairing $\int_{\mathcal{S}^{1}}(\bullet, \bullet)$ on $\mathfrak{g}$-valued differential forms) to invert one input in cyclic operations. This gives an oriented (non-cyclic) version of the unimodular $L_{\infty}$ structure (see e.g. [11]) on $\mathfrak{g} \otimes C^{\bullet}\left(\Xi_{n}\right)$ with structure maps $l_{k}^{(0)}: \wedge^{k}\left(\mathfrak{g} \otimes C^{\bullet}\left(\Xi_{n}\right)\right) \rightarrow \mathfrak{g} \otimes C^{\bullet}\left(\Xi_{n}\right)$ and $l_{k}^{(1)}: \wedge^{k}\left(\mathfrak{g} \otimes C^{\bullet}\left(\Xi_{n}\right)\right) \rightarrow \mathbb{R}$ 
related to the cyclic operations $c_{k}^{(l)}$ by the following formula:

$$
\begin{gathered}
c_{k+1}^{(0)}(\underbrace{\psi^{\prime}+A^{\prime}, \cdots, \psi^{\prime}+A^{\prime}}_{k+1})=\left(\psi^{\prime}+A^{\prime}, l_{k}^{(0)}(\underbrace{\psi^{\prime}+A^{\prime}, \cdots, \psi^{\prime}+A^{\prime}}_{k})\right), \quad k \geq 1 \\
c_{k}^{(1)}=l_{k}^{(1)}, \quad k \geq 2 .
\end{gathered}
$$

In this unimodular $L_{\infty}$ algebra, only the differential $l_{1}^{(0)}$ is local while all other operations (including the binary bracket $l_{2}^{(0)}: \wedge^{2}\left(\mathfrak{g} \otimes C^{\bullet}\left(\Xi_{n}\right)\right) \rightarrow \mathfrak{g} \otimes C^{\bullet}\left(\Xi_{n}\right)$ ) are non-local unlike in the simplicial BF theory [15] where all higher operations are simplicially-local.

Remark 12. The dressed chain homotopy $\kappa_{A^{\prime}}$ can be used to construct the non-linear $\operatorname{map} U: \mathcal{F}_{\Xi_{n}} \rightarrow \mathcal{F}$

$$
U: \psi^{\prime}+A^{\prime} \mapsto \psi^{\prime}+A^{\prime}-\kappa_{A^{\prime}} \operatorname{ad}_{A^{\prime}} \psi^{\prime} .
$$

It sends the infrared field $\psi^{\prime}+A^{\prime}$ to the conditional extremum of the continuum action $S$ restricted to $\left\{\psi^{\prime}+A^{\prime}\right\} \oplus \mathcal{L}$. The tree part of the simplicial action can be expressed in terms of $U$,

$$
\begin{aligned}
S_{\Xi_{n}}^{0} & =S\left(U\left(\psi^{\prime}+A^{\prime}\right)\right) \\
& =\int_{\mathcal{S}^{1}} \frac{1}{2}\left(U\left(\psi^{\prime}+A^{\prime}\right), d U\left(\psi^{\prime}+A^{\prime}\right)\right)+\frac{1}{6}\left(U\left(\psi^{\prime}+A^{\prime}\right),\left[U\left(\psi^{\prime}+A^{\prime}\right), U\left(\psi^{\prime}+A^{\prime}\right)\right]\right) .
\end{aligned}
$$

In the language of infinity algebras, $U$ is an $L_{\infty}$ morphism intertwining the DGLA structure on $\mathfrak{g} \otimes \Omega^{\bullet}\left(\mathcal{S}^{1}\right)$ and the $L_{\infty}$ structure on $\mathfrak{g} \otimes C^{\bullet}\left(\Xi_{n}\right)$.

Remark 13. There are two natural systems of $\mathfrak{g}$-valued coordinates on the space of simplicial BV fields $\mathcal{F}_{\Xi_{n}}:\left\{\psi_{k}, A_{k}\right\}$ and $\left\{\tilde{\psi}_{k}, A_{k}\right\}$ (where variables $\tilde{\psi}_{k}$ are defined by (46)). The first coordinate system is associated to the realization of the space of fields through the cochain complex of a triangulation: $\mathcal{F}_{\Xi_{n}}=\Pi \mathfrak{g} \otimes C^{\bullet}\left(\Xi_{n}\right)$. The second coordinate system is associated to the realization through 1-chains and 1-cochains: $\mathcal{F}_{\Xi_{n}} \cong$ $\mathfrak{g} \otimes C_{1}\left(\Xi_{n}\right) \oplus \Pi \mathfrak{g} \otimes C^{1}\left(\Xi_{n}\right)$ (or instead of 1-chains of $\Xi_{n}$ one can talk of 0-cochains of the dual cell decomposition $\left.\Xi_{n}^{\vee}: \mathcal{F}_{\Xi_{n}} \cong \Pi \mathfrak{g} \otimes C^{0}\left(\Xi_{n}^{\vee}\right) \oplus \Pi \mathfrak{g} \otimes C^{1}\left(\Xi_{n}\right)\right)$. The convenience of the first coordinate system is that the abelian part of the simplcial action (42) is local in variables $\left(\psi_{k}, A_{k}\right)$. The convenience of the second coordinate system $\left\{\tilde{\psi}_{k}, A_{k}\right\}$ is that the BV Laplacian becomes diagonal (45).

\section{Approach Through Operator Formalism}

In this section, our strategy is to give a new definition of the one-dimensional ChernSimons theory. It will be inspired by the definition of Sect. 2.1, but we will be able to consider our theory on an interval and to define a concatenation (gluing) procedure. We will check that the results obtained by the new approach are consistent with those of Sect. 2.

For the rest of the paper, we will assume that $\operatorname{dim} \mathfrak{g}=2 m$ is even. This is important for Theorem 2 (the correspondence between the operator and path integral formalism): its proof relies on recovering the path integral by using the fundamental representation of Clifford algebra $C l(\mathfrak{g})$ (Sect. 4.1) which is simpler for $\operatorname{dim} \mathfrak{g}=2 \mathrm{~m}$. 


\subsection{One-dimensional Chern-Simons theory in operator formalism.}

3.1.1. First approximation. We would like to define the one-dimensional Chern-Simons theory (8) as quantum mechanics where components of the quantized odd field $\left\{\hat{\psi}^{a}\right\}$ are subject to the anti-commutation relations

$$
\hat{\psi}^{a} \hat{\psi}^{b}+\hat{\psi}^{b} \hat{\psi}^{a}=\hbar \delta^{a b},
$$

i.e. $\left\{\hat{\psi}^{a}\right\}$ are generators of the Clifford algebra $C l(\mathfrak{g})$. The even 1-form (connection) field $A=d \tau T^{a} A^{a}(\tau)$ is non-dynamical, and it is treated as a classical background. The evolution operator for the theory on the interval is defined as a path-ordered exponential of $A$ in the spin representation:

$$
U_{\mathcal{I}}(A)=\overleftarrow{P \exp }\left(-\frac{1}{2 \hbar} \int_{\mathcal{I}} d \tau f^{a b c} \hat{\psi}^{a} A^{b}(\tau) \hat{\psi}^{c}\right) \in C l(\mathfrak{g})
$$

Here, the intuition is as follows: the term $\frac{1}{2} \int(\psi, d \psi)$ in the action (8) generates the canonical anti-commutation relations (47) while the term $\frac{1}{2} \int(\psi,[A, \psi])$ generates the time-dependent quantum Hamiltonian $\hat{H}(\tau)=-\frac{1}{2} f^{a b c} \hat{\psi}^{a} A^{b}(\tau) \hat{\psi}^{c}$ which appears in (48). The evolution operator for the concatenation of intervals $\mathcal{I}_{1}=\left[\mathfrak{p}_{1}, \mathfrak{p}_{2}\right], \mathcal{I}_{2}=$ $\left[\mathfrak{p}_{2}, \mathfrak{p}_{3}\right]$ with connections $A_{1}, A_{2}$ is naturally given by the product of the corresponding evolution operators in the Clifford algebra:

$$
U_{\left[\mathfrak{p}_{1}, \mathfrak{p}_{3}\right]}\left(A_{1} \theta_{\left[\mathfrak{p}_{1}, \mathfrak{p}_{2}\right]}+A_{2} \theta_{\left[\mathfrak{p}_{2}, \mathfrak{p}_{3}\right]}\right)=U_{\left[\mathfrak{p}_{2}, \mathfrak{p}_{3}\right]}\left(A_{2}\right) \cdot U_{\left[\mathfrak{p}_{1}, \mathfrak{p}_{2}\right]}\left(A_{1}\right) .
$$

As in Sect. 2.3.2, $\theta_{\left[\mathfrak{p}_{k}, \mathfrak{p}_{k+1}\right]}=\theta_{\mathcal{I}_{k}}$ denotes the function taking value 1 on the interval $\mathcal{I}_{k}$ and zero everywhere else. The partition function for a circle is given by

$$
Z_{\mathcal{S}^{1}}(A)=\operatorname{Str}_{C l(\mathfrak{g})} \overleftarrow{P \exp }\left(-\frac{1}{2 \hbar} \int_{\mathcal{I}} d \tau f^{a b c} \hat{\psi}^{a} A^{b}(\tau) \hat{\psi}^{c}\right) \in \mathbb{R}
$$

where $\operatorname{Str}_{C l(\mathfrak{g})}$ is the super-trace on $C l(\mathfrak{g})$ defined as

$$
\operatorname{Str}_{C l(\mathfrak{g})}: \quad \hat{a} \mapsto(i \hbar)^{m} \cdot\left(\text { Coefficient of } \hat{\psi}^{1} \cdots \hat{\psi}^{2 m} \text { in } \hat{a}\right) .
$$

3.1.2. Imposing the cyclic Whitney gauge. Our next task is to model in operator formalism the fiber BV integral (36) for the theory on the circle. We will look for the analogue of the cyclic Whitney gauge introduced in Sect. 2.3.1. For the connection $A$, imposing the gauge just amounts to saying that $A$ is now infrared, i.e. a piecewise-constant connection $A^{\prime}=\sum_{k=1}^{n} d \tau A_{k} \theta_{\mathcal{I}_{k}}$ with $A_{k} \in \mathfrak{g}$. For $\psi$, we would like to restrict the integration to $\psi$ 's with given integrals (average values) $\tilde{\psi}_{k}=\frac{\psi_{k}+\psi_{k+1}}{2}$ over intervals $\mathcal{I}_{k}$. So, we are interested in the integral

$$
\begin{aligned}
e^{\frac{1}{\hbar} S_{\Xi_{n}}} & =\int \mathcal{D} \psi e^{\frac{1}{2 \hbar} \int_{\mathcal{S}^{1}}\left((\psi, d \psi)+\left(\psi, \mathrm{ad}_{A^{\prime}} \psi\right)\right)} \prod_{k=1}^{n} \delta\left(\int_{\mathcal{I}_{k}} d \tau \psi-\tilde{\psi}_{k}\right) \\
& =\int \prod_{k=1}^{n} D \lambda_{k} e^{-\sum_{k=1}^{n}\left(\lambda_{k}, \tilde{\psi}_{k}\right)} \underbrace{\int \mathcal{D} \psi e^{\frac{1}{2 \hbar} \int_{\mathcal{S}^{1}}\left((\psi, d \psi)+\left(\psi, \mathrm{ad}_{A^{\prime}} \psi\right)\right)+\sum_{k=1}^{n}\left(\lambda_{k}, \psi\right)}}_{Z_{\Xi_{n}}\left(\lambda^{\prime}, A^{\prime}\right)} .
\end{aligned}
$$


Here we got rid of $\delta$-functions at the cost of introducing odd auxiliary variables $\lambda_{k}=$ $T^{a} \lambda_{k}^{a} \in \Pi \mathfrak{g}$. One can organize them into an odd piecewise-constant function on the circle $\lambda^{\prime}=\sum_{k=1}^{n} \lambda_{k} \theta_{\mathcal{I}_{k}}$ which plays the role of a source for the field $\psi$. The entity $Z_{\Xi_{n}}\left(\lambda^{\prime}, A^{\prime}\right)$ that appeared in the integrand can be written in the operator formalism as

$$
Z_{\Xi_{n}}\left(\lambda^{\prime}, A^{\prime}\right)=\operatorname{Str}_{C l(\mathfrak{g})} \prod_{k=1}^{\overleftarrow{n}} \exp \left(-\frac{1}{2 \hbar} f^{a b c} \hat{\psi}^{a} A_{k}^{b} \hat{\psi}^{c}+\lambda_{k}^{a} \hat{\psi}^{a}\right)
$$

Then, the partition function of the one-dimensional Chern-Simons theory on the circle (in the Whitney gauge) is given by the odd Fourier transform of (51):

$$
Z_{\Xi_{n}}\left(\psi^{\prime}, A^{\prime}\right)=(i \hbar)^{-n m} \int \prod_{k=1}^{n} D \lambda_{k} e^{-\sum_{k=1}^{n} \lambda_{k}^{a} \tilde{\psi}_{k}^{a}} Z_{\Xi_{n}}\left(\lambda^{\prime}, A^{\prime}\right) .
$$

Remark 14. To be precise with signs, we should introduce an ordering convention for the Berezin measure in (52). We set

$$
\prod_{k=1}^{n} D \lambda_{k}=\prod_{k=1}^{n} \vec{\prod}_{a=1}^{2 m} D \lambda_{k}^{a}
$$

Theorem 2. For $n$ odd and $\operatorname{dim} \mathfrak{g}=2 m$ even, one has

$$
Z_{\Xi_{n}}\left(\psi^{\prime}, A^{\prime}\right)=e^{\frac{1}{\hbar} S_{\Xi_{n}}\left(\psi^{\prime}, A^{\prime}\right)},
$$

where the right hand side is given by (42) and the left hand side is defined by (51, 52).

We will prove this theorem in Sect. 4.4 .2 by constructing a path integral representation for $Z_{\Xi_{n}}$. But first (see Sects. 3.1.3, 3.1.4) we will perform some direct tests of formula (53).

Remark 15. Note that we can define the right-hand side of (53) only for $n$ odd while the definition of left-hand side makes sense for both even and odd $n$. A simple computation shows that for $n$ even the partition function $Z_{\Xi_{n}}\left(\psi^{\prime}, A^{\prime}\right)$ vanishes at $\psi^{\prime}=0, A^{\prime}=0$. This agrees with the observation that for $n$ even the Whitney gauge does not apply (see Sect. 2).

Remark 16. We chose the normalization for the super-trace in Clifford algebra (49) and for $Z_{\Xi_{n}}$ (52) in a way consistent with the path integral formalism (see (76), (77)).

3.1.3. Consistency check: the effective action on cohomology. 9 The first test of the correspondence (53) is the case of $n=1$. Let us first compute the following expression in the Clifford algebra with two generators $\mathrm{Cl}_{2}$ :

$$
\begin{aligned}
& \varphi\left(\tilde{\psi}^{1}, \tilde{\psi}^{2}, a\right) \\
& =(i \hbar)^{-1} \int D \lambda^{1} D \lambda^{2} e^{-\lambda^{1} \tilde{\psi}^{1}-\lambda^{2} \tilde{\psi}^{2}} \operatorname{Str}_{C l_{2}} \exp \left(-\frac{1}{\hbar} \hat{\psi}^{1} a \hat{\psi}^{2}+\lambda^{1} \hat{\psi}^{1}+\lambda^{2} \hat{\psi}^{2}\right),
\end{aligned}
$$

\footnotetext{
9 For the reader's convenience, we present explicit calculations in the Clifford algebra here and in subsequent sections; the general reference for the Clifford calculus is [4].
} 
where $a \in \mathbb{R}$ is a number. For the exponential under the super-trace we have

$$
\begin{aligned}
\exp \left(-\frac{1}{\hbar} \hat{\psi}^{1} a \hat{\psi}^{2}+\lambda^{1} \hat{\psi}^{1}+\lambda^{2} \hat{\psi}^{2}\right)= & \left(-\frac{2}{\hbar} \sin (a / 2)-\frac{\sin (a / 2)}{a / 2} \lambda^{1} \lambda^{2}\right) \hat{\psi}^{1} \hat{\psi}^{2} \\
& +\frac{\sin (a / 2)}{a / 2}\left(\lambda^{1} \hat{\psi}^{1}+\lambda^{2} \hat{\psi}^{2}\right) \\
& +\left(\cos (a / 2)+\hbar \frac{1}{a}\left(\cos (a / 2)-\frac{\sin (a / 2)}{a / 2}\right) \lambda^{1} \lambda^{2}\right)
\end{aligned}
$$

In this expression, only the first term contributes to the super-trace in (54), and we obtain

$$
\begin{aligned}
\varphi\left(\tilde{\psi}^{1}, \tilde{\psi}^{2}, a\right) & =\int D \lambda^{1} D \lambda^{2} e^{-\lambda^{1} \tilde{\psi}^{1}-\lambda^{2} \tilde{\psi}^{2}}\left(-\frac{2}{\hbar} \sin (a / 2)-\frac{\sin (a / 2)}{a / 2} \lambda^{1} \lambda^{2}\right) \\
& =\frac{\sin (a / 2)}{a / 2}-\frac{2}{\hbar} \sin (a / 2) \tilde{\psi}^{1} \tilde{\psi}^{2}=\frac{\sin (a / 2)}{a / 2} e^{-\frac{1}{\hbar} \tilde{\psi}^{1} a \tilde{\psi}^{2}} .
\end{aligned}
$$

Next let us consider an anti-symmetric block-diagonal matrix with blocks $2 \times 2$

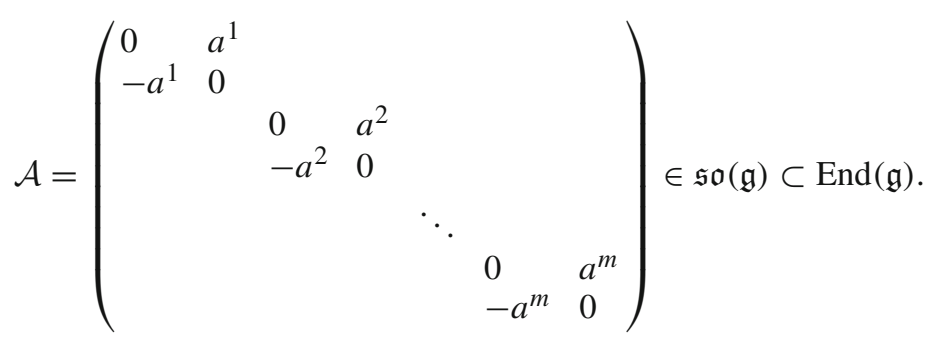

Then, we have

$$
\begin{aligned}
(i \hbar)^{-m} \int \prod_{a} D \lambda^{a} e^{-\lambda^{a} \tilde{\psi}^{a}} \operatorname{Str}_{C l(\mathfrak{g})} \exp \left(-\frac{1}{2 \hbar} \hat{\psi}^{a} \mathcal{A}^{a b} \hat{\psi}^{b}+\lambda^{a} \hat{\psi}^{a}\right) \\
=\prod_{p=1}^{m} \varphi\left(\tilde{\psi}^{2 p-1}, \tilde{\psi}^{2 p}, a^{p}\right)=\operatorname{det}_{g}^{1 / 2}\left(\frac{\sinh \frac{\mathcal{A}}{2}}{\frac{\mathcal{A}}{2}}\right) \cdot e^{-\frac{1}{2 \hbar} \tilde{\psi}^{a} \mathcal{A}^{a b} \tilde{\psi}^{b}} .
\end{aligned}
$$

Since both the left-hand side and the right hand side of (57) are $S O(\mathfrak{g})$-invariant, the equality actually holds for all anti-symmetric matrices $\mathcal{A}$, and in particular for $\mathcal{A}=\operatorname{ad}_{A_{0}}$, where $A_{0} \in \mathfrak{g}$. Thus, we have shown that

$$
Z_{\Xi_{1}}\left(\tilde{\psi}, A_{0}\right)=\operatorname{det}_{g}^{1 / 2}\left(\frac{\sinh \frac{\operatorname{ad}_{A_{0}}}{2}}{\frac{\operatorname{ad}_{A_{0}}}{2}}\right) \cdot e^{-\frac{1}{2 \hbar}\left(\tilde{\psi}, \operatorname{ad}_{A_{0}} \tilde{\psi}\right)}
$$

The right-hand side coincides with (13), so we have checked the correspondence (53) in the case of $n=1$. 
3.1.4. Consistency check: the case of mutually commuting $\left\{A_{k}\right\}$ and $\psi^{\prime}=0$. Now we would like to perform a direct check of the correspondence (53) at the point $\psi^{\prime}=0$ (i.e. neglecting the tree part of the simplicial action) and assuming that $\left[A_{k}, A_{k^{\prime}}\right]=0$ for all $k, k^{\prime}=1, \ldots, n$. That is, we will check that

$$
\begin{aligned}
& (i \hbar)^{-n m} \int \prod_{k=1}^{n} D \lambda_{k} \operatorname{Str}_{C l(\mathfrak{g})} \overleftarrow{\prod_{k=1}^{n}} \exp \left(-\frac{1}{2 \hbar} f^{a b c} \hat{\psi}^{a} A_{k}^{b} \hat{\psi}^{c}+\lambda_{k}^{a} \hat{\psi}^{a}\right) \\
& =\operatorname{det}_{\mathfrak{g}}^{1 / 2}\left(\frac{1+\prod_{k=1}^{n} R\left(\operatorname{ad}_{A_{k}}\right)}{\prod_{k=1}^{n}\left(1+R\left(\operatorname{ad}_{A_{k}}\right)\right)} \cdot \prod_{k=1}^{n} \frac{\sinh \frac{\operatorname{ad}_{A_{k}}}{2}}{\frac{\operatorname{ad}_{A_{k}}}{2}}\right) .
\end{aligned}
$$

We use the idea of Sect. 3.1.3 to reduce (58) to a computation in $C l_{2}$. Since all $A_{k}$ mutually commute, we can choose an orthonormal basis in $\mathfrak{g}$, such that the matrices $\operatorname{ad}_{A_{k}}$ simultaneously assume the standard form (56). Then, both sides of (58) factorize into contributions of $2 \times 2$ blocks, and it suffices to check the identity

$$
\begin{aligned}
& (i \hbar)^{-n} \int \prod_{k=1}^{n}\left(D \lambda_{k}^{1} D \lambda_{k}^{2}\right) \operatorname{Str}_{C l_{2}} \prod_{k=1}^{n} \exp \left(-\frac{1}{\hbar} \hat{\psi}^{1} a_{k} \hat{\psi}^{2}+\lambda_{k}^{1} \hat{\psi}^{1}+\lambda_{k}^{2} \hat{\psi}^{2}\right) \\
& =\operatorname{det}^{1 / 2}\left(\frac{1+\prod_{k=1}^{n} R\left(i \sigma_{2} a_{k}\right)}{\prod_{k=1}^{n}\left(1+R\left(i \sigma_{2} a_{k}\right)\right)} \cdot \prod_{k=1}^{n} \frac{\sinh \frac{i \sigma_{2} a_{k}}{2}}{\frac{i \sigma_{2} a_{k}}{2}}\right) .
\end{aligned}
$$

Here $\sigma_{2}=\left(\begin{array}{cc}0 & -i \\ i & 0\end{array}\right)$ is the second Pauli matrix, and $i \sigma_{2} a_{k}=\left(\begin{array}{cc}0 & a_{k} \\ -a_{k} & 0\end{array}\right)$. To evaluate the left-hand side of (59), we use the result (55) for the Clifford exponential:

1.h.s of $(59)=(i \hbar)^{-n} \operatorname{Str}_{C l_{2}} \prod_{k=1}^{\overleftarrow{n}}\left(\frac{\sin \left(a_{k} / 2\right)}{a_{k} / 2} \hat{\psi}^{1} \hat{\psi}^{2}+\hbar \frac{1}{a_{k}}\left(\frac{\sin \left(a_{k} / 2\right)}{a_{k} / 2}-\cos \left(a_{k} / 2\right)\right)\right)$

An easy way to evaluate this expression is to use the matrix representation $\mathrm{Cl}_{2} \rightarrow$ $\operatorname{End}\left(\mathbb{C}^{1 \mid 1}\right)$ which maps

$$
\hat{\psi}^{1} \mapsto\left(\frac{\hbar}{2}\right)^{1 / 2}\left(\frac{0 \mid 1}{1 \mid 0}\right), \quad \hat{\psi}^{2} \mapsto\left(\frac{\hbar}{2}\right)^{1 / 2}\left(\frac{0 \mid-i}{i \mid 0}\right), \quad \operatorname{Str}_{C l_{2}} \mapsto \operatorname{Str}_{\operatorname{End}\left(\mathbb{C}^{1 \mid 1}\right)},
$$

and

$$
\operatorname{Str}_{\operatorname{End}\left(\mathbb{C}^{1 \mid 1}\right)}:\left(\begin{array}{c|c}
\alpha & \beta \\
\hline \gamma \mid \delta
\end{array}\right) \mapsto \alpha-\delta
$$

is the standard super-trace on matrices of the size $(1 \mid 1) \times(1 \mid 1)$. Using this representation, we obtain

$$
\begin{aligned}
& \text { 1.h.s of (59) } \\
& =i^{-n} \operatorname{Str}_{\operatorname{End}\left(\mathbb{C}^{1 \mid 1}\right)} \prod_{k=1}^{n}\left(\frac{\frac{1}{a_{k}}\left(\frac{\sin \left(a_{k} / 2\right)}{a_{k} / 2}-\cos \left(a_{k} / 2\right)\right)+\frac{i}{2} \frac{\sin \left(a_{k} / 2\right)}{a_{k} / 2} \mid 0}{0} \mid \frac{1}{a_{k}}\left(\frac{\sin \left(a_{k} / 2\right)}{a_{k} / 2}-\cos \left(a_{k} / 2\right)\right)-\frac{i}{2} \frac{\sin \left(a_{k} / 2\right)}{a_{k} / 2}\right) \\
& =i^{-n} \prod_{k=1}^{n} \frac{\sin \left(a_{k} / 2\right)}{a_{k} / 2} \cdot 2 i \operatorname{Im} \prod_{k=1}^{n}\left(\frac{i}{2}+\frac{1}{a_{k}}-\frac{1}{2} \cot \left(a_{k} / 2\right)\right) \\
& =2 \prod_{k=1}^{n} \frac{\sin \left(a_{k} / 2\right)}{a_{k} / 2} \cdot \operatorname{Re} \prod_{k=1}^{n} \frac{1}{1+R\left(i a_{k}\right)} .
\end{aligned}
$$

In the last line we used the assumption that $n$ is odd. 
To evaluate the right-hand side of (59), first observe that the matrix

$$
\frac{1+\prod_{k=1}^{n} R\left(i \sigma_{2} a_{k}\right)}{\prod_{k=1}^{n}\left(1+R\left(i \sigma_{2} a_{k}\right)\right)}=\frac{1}{\prod_{k=1}^{n}\left(1+R\left(i \sigma_{2} a_{k}\right)\right)}+\frac{1}{\prod_{k=1}^{n}\left(1+R\left(-i \sigma_{2} a_{k}\right)\right)}
$$

is constructed from matrices $i \sigma_{2} a_{k}=\left(\begin{array}{cc}0 & a_{k} \\ -a_{k} & 0\end{array}\right)$ and is therefore of form $\left(\begin{array}{cc}\alpha & \beta \\ -\beta & \alpha\end{array}\right)$ (i.e. belongs to $\operatorname{Span}_{\mathbb{R}}\left(1, i \sigma_{2}\right)$ ), and that it is symmetric. Hence, it is actually a multiple of the identity matrix $\left(\begin{array}{ll}\alpha & 0 \\ 0 & \alpha\end{array}\right)$, and the determinant may be expressed as

$$
\begin{aligned}
\operatorname{det}^{1 / 2}\left(\frac{1+\prod_{k=1}^{n} R\left(i \sigma_{2} a_{k}\right)}{\prod_{k=1}^{n}\left(1+R\left(i \sigma_{2} a_{k}\right)\right)}\right) & =\frac{1}{2} \operatorname{tr}\left(\frac{1+\prod_{k=1}^{n} R\left(i \sigma_{2} a_{k}\right)}{\prod_{k=1}^{n}\left(1+R\left(i \sigma_{2} a_{k}\right)\right)}\right) \\
& =\prod_{k=1}^{n} \frac{1}{1+R\left(i a_{k}\right)}+\prod_{k=1}^{n} \frac{1}{1+R\left(-i a_{k}\right)} \\
& =2 \operatorname{Re} \prod_{k=1}^{n} \frac{1}{1+R\left(i a_{k}\right)}
\end{aligned}
$$

Now it is obvious that (59) is verified, and this implies (58).

\subsection{One-dimensional Chern-Simons with boundary.}

3.2.1. One-dimensional simplicial Chern-Simons in the operatorformalism. Concatenations. There is a natural construction of the one-dimensional simplicial Chern-Simons theory in the operator formalism: to a one-dimensional oriented simplicial complex $\Theta$ (a collection of $i(\Theta)$ triangulated intervals and $c(\Theta)$ circles) it associates a partition function

$$
Z_{\Theta} \in \operatorname{Fun}(\underbrace{\mathfrak{g} \otimes C_{1}(\Theta) \oplus \Pi \mathfrak{g} \otimes C^{1}(\Theta)}_{\mathcal{F}_{\Theta}^{\text {bulk }}}) \otimes C l(\mathfrak{g})^{\otimes i(\Theta)},
$$

where we think of variables $A_{k} \in \mathfrak{g}$ as coordinates on $\mathfrak{g}$-valued simplicial 1-cochains of $\Theta$ with parity shifted, and we think of variables $\tilde{\psi}_{k} \in \Pi \mathfrak{g}$ as coordinates on $\mathfrak{g}$-valued simplicial 1-chains of $\Theta$. The partition function $Z_{\Theta}$ is defined by the following properties:

- For a single interval with a standard triangulation, we have

$$
Z_{\mathcal{I}}=(i \hbar)^{-m} \int \prod_{a=1}^{2 m} D \lambda^{a} e^{-\lambda^{a} \tilde{\psi}^{a}} \exp \left(-\frac{1}{2 \hbar} f^{a b c} \hat{\psi}^{a} A^{b} \hat{\psi}^{c}+\lambda^{a} \hat{\psi}^{a}\right)
$$

- For a disjoint union of $\Theta_{1}$ and $\Theta_{2}$,

$$
Z_{\Theta_{1} \sqcup \Theta_{2}}=Z_{\Theta_{1}} \otimes Z_{\Theta_{2}} .
$$


- For a concatenation $\Theta_{1} \cup \Theta_{2}$ with intersection $\Theta_{1} \cap \Theta_{2}=\mathfrak{p}$ being a single point embedded as a boundary point of positive orientation $\mathfrak{p}_{1} \in \Theta_{1}$ belonging to the $i^{\text {th }}$ triangulated interval of $\Theta_{1}$, and embedded as a boundary point of negative orientation $\mathfrak{p}_{2} \in \Theta_{2}$ belonging to the $j^{\text {th }}$ triangulated interval of $\Theta_{2}$, we have:

$$
Z_{\Theta_{1} \cup \Theta_{2}}=\mathfrak{m}\left(Z_{\Theta_{2}} \otimes Z_{\Theta_{1}}\right) .
$$

Here $\mathfrak{m}: C l(\mathfrak{g}) \otimes C l(\mathfrak{g}) \rightarrow C l(\mathfrak{g})$ is the Clifford algebra multiplication, and $\mathfrak{m}$ in (62) acts on Clifford algebras associated to the $j^{\text {th }}$ triangulated interval of $\Theta_{2}$ and the $i^{\text {th }}$ triangulated interval of $\Theta_{1}$.

- If the simplicial complex $\Theta^{\prime}$ is obtained from $\Theta$ by closing the $i^{\text {th }}$ triangulated interval into a circle, we have:

$$
Z_{\Theta^{\prime}}=\operatorname{Str}_{C l(\mathfrak{g})} Z_{\Theta},
$$

where the super-trace is taken over the Clifford algebra associated to the $i^{\text {th }}$ interval of $\Theta$.

Obviously, this construction gives (52) for a triangulated circle $\Theta=\Xi_{n}$, and due to the correspondence (53) it is consistent with the results of Sect. 2.

Remark 17 . We may regard $Z_{\mathcal{I}}$ as a contribution of an open interval. Then, the concatenation (62) is understood as taking a disjoint union of open triangulated intervals and then gluing them together at the point $\mathfrak{p}$. Likewise, (63) is understood as gluing together two end-points of the same interval. Thus, a contribution of point is either the $(1,2)-$ tensor ${ }^{10} \mathfrak{m}$ on the Clifford algebra or the $(0,1)$-tensor $\operatorname{Str}_{C l(\mathfrak{g})}$, depending on whether we are gluing together two different or the same connected component.

Remark 18. Once we come to a description of the one-dimensional Chern-Simons theory in terms of Atiyah-Segal Axioms and specify the vector space associated to a point (the space of states), properties (62) and (63) become a single sewing axiom (see Sect. 4.2 below).

3.2.2. Simplicial aggregations. Let $\Theta^{\prime}$ be the interval $\left[\mathfrak{p}_{1}, \mathfrak{p}_{3}\right]$ with the standard triangulation, and let $\Theta$ be the subdivision of $\Theta^{\prime}$ with three 0 -simplices $\mathfrak{p}_{1}, \mathfrak{p}_{2}, \mathfrak{p}_{3}$ and two 1 -simplices $\left[\mathfrak{p}_{1}, \mathfrak{p}_{2}\right],\left[\mathfrak{p}_{2}, \mathfrak{p}_{3}\right]$. The aggregation morphism $r^{\xi}$ acts on simplicial chains as

$$
\begin{aligned}
r_{C_{\bullet}}^{\xi}: & C_{\bullet}(\Theta) \rightarrow C_{\bullet}\left(\Theta^{\prime}\right) \\
& \alpha_{\mathfrak{p}_{1}} e^{\mathfrak{p}_{1}}+\alpha_{\mathfrak{p}_{2}} e^{\mathfrak{p}_{2}}+\alpha_{\mathfrak{p}_{3}} e^{\mathfrak{p}_{3}}+\alpha_{\mathfrak{p}_{1} \mathfrak{p}_{2}} e^{\mathfrak{p}_{1} \mathfrak{p}_{2}}+\alpha_{\mathfrak{p}_{2} \mathfrak{p}_{3}} e^{\mathfrak{p}_{2} \mathfrak{p}_{3}} \\
& \mapsto\left(\alpha_{\mathfrak{p}_{1}}+(1-\xi) \alpha_{\mathfrak{p}_{2}}\right) e^{\prime \mathfrak{p}_{1}}+\left(\alpha_{\mathfrak{p}_{3}}+\xi \alpha_{\mathfrak{p}_{2}}\right) e^{\prime \mathfrak{p}_{3}}+\left(\xi \alpha_{\mathfrak{p}_{1} \mathfrak{p}_{2}}+(1-\xi) \alpha_{\mathfrak{p}_{2} \mathfrak{p}_{3}}\right) e^{\prime \mathfrak{p}_{1} \mathfrak{p}_{3}},
\end{aligned}
$$

and on simplicial cochains as

$$
\begin{aligned}
& r_{C^{\bullet}}^{\xi}: C^{\bullet}(\Theta) \rightarrow C^{\bullet}\left(\Theta^{\prime}\right) \\
& \alpha^{\mathfrak{p}_{1}} e_{\mathfrak{p}_{1}}+\alpha^{\mathfrak{p}_{2}} e_{\mathfrak{p}_{2}}+\alpha^{\mathfrak{p}_{3}} e_{\mathfrak{p}_{3}}+\alpha^{\mathfrak{p}_{1} \mathfrak{p}_{2}} e_{\mathfrak{p}_{1} \mathfrak{p}_{2}}+\alpha^{\mathfrak{p}_{2} \mathfrak{p}_{3}} e_{\mathfrak{p}_{2} \mathfrak{p}_{3}} \\
& \mapsto \alpha^{\mathfrak{p}_{1}} e_{\mathfrak{p}_{1}}^{\prime}+\alpha^{\mathfrak{p}_{3}} e_{\mathfrak{p}_{3}}^{\prime}+\left(\alpha^{\mathfrak{p}_{1} \mathfrak{p}_{2}}+\alpha^{\mathfrak{p}_{2} \mathfrak{p}_{3}}\right) e_{\mathfrak{p}_{1} \mathfrak{p}_{3}}^{\prime} .
\end{aligned}
$$

Here $0<\xi<1$ is a parameter determining the relative weight of intervals $\left[\mathfrak{p}_{1}, \mathfrak{p}_{2}\right]$ and $\left[\mathfrak{p}_{2}, \mathfrak{p}_{3}\right]$ inside $\left[\mathfrak{p}_{1}, \mathfrak{p}_{3}\right]$ (the symmetric choice corresponds to $\xi=1 / 2$ ). Basis chains

10 We mean the rank of the tensor: one time covariant, two times contravariant. 
and cochains corresponding a simplex $\sigma$ are denoted by $e^{\sigma}$ and $e_{\sigma}$, respectively. We use primes to distinguish the basis of $C_{\bullet}\left(\Theta^{\prime}\right), C^{\bullet}\left(\Theta^{\prime}\right) ; \alpha_{\ldots}, \alpha^{\cdots}$ are numerical coefficients.

One can also introduce the subdivision morphism which is dual to the aggregation morphism:

$$
i_{C_{\bullet}}^{\xi}=\left(r_{C^{\bullet}}^{\xi}\right)^{*}: C_{\bullet}\left(\Theta^{\prime}\right) \rightarrow C_{\bullet}(\Theta), \quad i_{C^{\bullet}}^{\xi}=\left(r_{C_{\bullet}}^{\xi}\right)^{*}: C^{\bullet}\left(\Theta^{\prime}\right) \rightarrow C^{\bullet}(\Theta)
$$

More explicitly,

$$
\begin{aligned}
i_{C \bullet}^{\xi}: & \alpha_{\mathfrak{p}_{1}}^{\prime} e^{\prime \mathfrak{p}_{1}}+\alpha_{\mathfrak{p}_{3}}^{\prime} e^{\prime \mathfrak{p}_{3}}+\alpha_{\mathfrak{p}_{1} \mathfrak{p}_{3}}^{\prime} e^{\prime \mathfrak{p}_{1} \mathfrak{p}_{3}} \mapsto \alpha_{\mathfrak{p}_{1}}^{\prime} e^{\mathfrak{p}_{1}}+\alpha_{\mathfrak{p}_{3}}^{\prime} e^{\mathfrak{p}_{3}}+\alpha_{\mathfrak{p}_{1} \mathfrak{p}_{3}}^{\prime}\left(e^{\mathfrak{p}_{1} \mathfrak{p}_{2}}+e^{\mathfrak{p}_{2} \mathfrak{p}_{3}}\right), \\
i_{C}^{\xi}: & \alpha^{\prime \mathfrak{p}_{1}} e_{\mathfrak{p}_{1}}^{\prime}+\alpha^{\prime \mathfrak{p}_{3}} e_{\mathfrak{p}_{3}}^{\prime}+\alpha^{\prime \mathfrak{p}_{1} \mathfrak{p}_{3}} e_{\mathfrak{p}_{1} \mathfrak{p}_{3}}^{\prime} \\
& \mapsto \alpha^{\prime \mathfrak{p}_{1}} e_{\mathfrak{p}_{1}}+\left((1-\xi) \alpha^{\prime \mathfrak{p}_{1}}+\xi \alpha^{\prime \mathfrak{p}_{3}}\right) e_{\mathfrak{p}_{2}}+\alpha^{\prime \mathfrak{p}_{3}} e_{\mathfrak{p}_{3}}+\alpha^{\prime \mathfrak{p}_{1} \mathfrak{p}_{3}}\left(\xi e_{\mathfrak{p}_{1} \mathfrak{p}_{2}}+(1-\xi) e_{\mathfrak{p}_{2} \mathfrak{p}_{3}}\right) .
\end{aligned}
$$

Aggregation and subdivision morphisms are chain maps. Moreover, they are quasiisomorphisms.

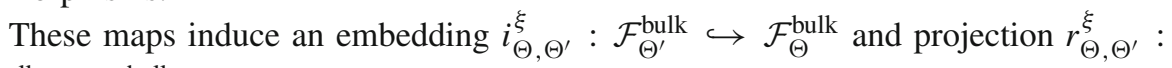
$\mathcal{F}_{\Theta}^{\text {bulk }} \rightarrow \mathcal{F}_{\Theta^{\prime}}^{\text {bulk }}$ for the spaces of "bulk fields",

$$
\mathcal{F}_{\Theta}^{\text {bulk }}=\mathfrak{g} \otimes C_{1}(\Theta) \oplus \Pi \mathfrak{g} \otimes C^{1}(\Theta), \quad \mathcal{F}_{\Theta^{\prime}}^{\text {bulk }}=\mathfrak{g} \otimes C_{1}\left(\Theta^{\prime}\right) \oplus \Pi \mathfrak{g} \otimes C^{1}\left(\Theta^{\prime}\right) .
$$

That is, we have a splitting

$$
\mathcal{F}_{\Theta}^{\text {bulk }}=i_{\Theta, \Theta^{\prime}}^{\xi}\left(\mathcal{F}_{\Theta^{\prime}}^{\text {bulk }}\right) \oplus \mathcal{F}_{\Theta, \Theta^{\prime}}^{\text {'bulk }, \xi}
$$

In more detail, we split the bulk fields as

$$
\tilde{\psi}_{1}=\tilde{\psi}^{\prime}+(1-\xi) \tilde{\psi}^{\prime \prime}, \quad \tilde{\psi}_{2}=\tilde{\psi}^{\prime}-\xi \tilde{\psi}^{\prime \prime}, \quad A_{1}=\xi A^{\prime}-A^{\prime \prime}, \quad A_{2}=(1-\xi) A^{\prime}+A^{\prime \prime} .
$$

The maps $i_{\Theta, \Theta^{\prime}}^{\xi}$ and $r_{\Theta, \Theta^{\prime}}^{\xi}$ are dual to each other with respect to the odd pairing on $\mathcal{F}_{\Theta}^{\text {bulk }}$ and $\mathcal{F}_{\Theta^{\prime}}^{\text {bulk }}$ (thus, (64) is an orthogonal decomposition). We denote by $\mathcal{L}_{\Theta, \Theta^{\prime}}^{\xi} \subset \mathcal{F}_{\Theta, \Theta^{\prime}}^{\prime \prime \text { bul }, \xi}$ the Lagrangian subspace defined by setting to zero the 1-cochain part of the ultraviolet field $A^{\prime \prime}$.

We define the action of the aggregation map $\Theta \rightarrow \Theta^{\prime}$ on the partition function of the one-dimensional simplicial Chern-Simons theory by the fiber BV integral associated to the splitting (64):

$$
\left(r_{\Theta, \Theta^{\prime}}^{\xi}\right)_{*}\left(Z_{\Theta}\right)=\int_{\mathcal{L}_{\Theta, \Theta^{\prime}}^{\xi}} Z_{\Theta} \in \operatorname{Fun}\left(\mathcal{F}_{\Theta^{\prime}}^{\text {bulk }}\right) \otimes C l(\mathfrak{g}) .
$$

It is easy to give a direct check of the following statement.

\section{Lemma 3.}

$$
\left(r_{\Theta, \Theta^{\prime}}^{\xi}\right)_{*}\left(Z_{\Theta}\right)=Z_{\Theta^{\prime}}
$$


Proof. Indeed, by definition (65) we have

$$
\begin{aligned}
& \left(r_{\Theta, \Theta^{\prime}}^{\xi}\right)_{*}\left(Z_{\Theta}\right)
\end{aligned}
$$

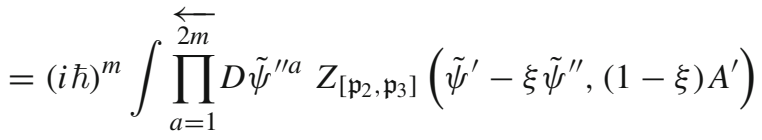

$$
\begin{aligned}
& \cdot Z_{\left[\mathfrak{p}_{1}, \mathfrak{p}_{2}\right]}\left(\tilde{\psi}^{\prime}+(1-\xi) \tilde{\psi}^{\prime \prime}, \xi A^{\prime}\right) \\
& =(i \hbar)^{-m} \int \prod_{a=1}^{\overleftarrow{2 m}} D \tilde{\psi}^{\prime \prime a} \prod_{a=1}^{\overrightarrow{2 m}} D \lambda_{2}^{a} \prod_{a=1}^{\overrightarrow{2 m}} D \lambda_{1}^{a} e^{-\lambda_{1}^{a}\left(\tilde{\psi}^{\prime a}+(1-\xi) \tilde{\psi}^{\prime \prime a}\right)-\lambda_{2}^{a}\left(\tilde{\psi}^{\prime a}-\xi \tilde{\psi}^{\prime \prime a}\right)} \\
& \cdot \exp \left(-\frac{1-\xi}{2 \hbar} f^{a b c} \hat{\psi}^{a} A^{\prime b} \hat{\psi}^{c}+\lambda_{2}^{a} \hat{\psi}^{a}\right) \cdot \exp \left(-\frac{\xi}{2 \hbar} f^{a b c} \hat{\psi}^{a} A^{\prime b} \hat{\psi}^{c}+\lambda_{1}^{a} \hat{\psi}^{a}\right) \text {. }
\end{aligned}
$$

Here we made a change of coordinates $\left(\lambda_{1}, \lambda_{2}\right) \rightarrow\left(\lambda=\lambda_{1}+\lambda_{2}, v=(1-\xi) \lambda_{1}-\xi \lambda_{2}\right)$. The integral over $\tilde{\psi}^{\prime \prime}$ produces the delta-function $\delta(v)$; by integrating over $v$ we obtain

$$
\begin{aligned}
\left(r_{\Theta, \Theta^{\prime}}^{\xi}\right)_{*}\left(Z_{\Theta}\right)= & (i \hbar)^{-m} \int \prod_{a=1}^{\overrightarrow{2 m}} D \lambda^{a} e^{-\lambda^{a} \tilde{\psi}^{\prime a}} . \\
& \cdot \exp \left(-\frac{1-\xi}{2 \hbar} f^{a b c} \hat{\psi}^{a} A^{\prime b} \hat{\psi}^{c}+(1-\xi) \lambda^{a} \hat{\psi}^{a}\right) \\
& \cdot \exp \left(-\frac{\xi}{2 \hbar} f^{a b c} \hat{\psi}^{a} A^{\prime b} \hat{\psi}^{c}+\xi \lambda^{a} \hat{\psi}^{a}\right) \\
= & (i \hbar)^{-m} \int \prod_{a=1}^{\overrightarrow{2 m} D \lambda^{a} e^{-\lambda^{a}} \tilde{\psi}^{\prime a}} \\
& \cdot \exp \left(-\frac{1}{2 \hbar} f^{a b c} \hat{\psi}^{a} A^{\prime b} \hat{\psi}^{c}+\lambda^{a} \hat{\psi}^{a}\right)=Z_{\Theta^{\prime}} .
\end{aligned}
$$

Remark 19. We normalize the measure on $\mathcal{L}_{\Theta, \Theta^{\prime}}^{\xi}$ in such a way that relation (66) holds with no additional factors.

Up to now, we only discussed the elementary aggregation which takes an interval subdivided into two smaller intervals and into an interval with the standard triangulation (that is, one removes the middle point $\mathfrak{p}_{2}$ and merges intervals $\left[\mathfrak{p}_{1}, \mathfrak{p}_{2}\right]$ and $\left[\mathfrak{p}_{2}, \mathfrak{p}_{3}\right]$ ). A general simplicial aggregation for a one-dimensional simplicial complex is a sequence of elementary aggregations made at each step on an incident pair of intervals. In particular, there are many simplicial aggregations for triangulated circles: $\Xi_{n} \rightarrow \Xi_{n^{\prime}}$ with $n>n^{\prime}$.

The following is an immediate consequence of (66):

Proposition 2. For a general simplicial aggregation

$$
r=r_{\Theta_{l-1}, \Theta^{\prime}}^{\xi_{l}} \circ \cdots \circ r_{\Theta_{1}, \Theta_{2}}^{\xi_{2}} \circ r_{\Theta, \Theta_{1}}^{\xi_{1}}: \Theta \rightarrow \Theta^{\prime}
$$


(where $\Theta$ is an arbitrary one-dimensional simplicial complex and $\Theta^{\prime}$ is some aggregation of $\Theta$ ), one has

$$
r_{*}\left(Z_{\Theta}\right)=Z_{\Theta^{\prime}} .
$$

The compatibility with aggregations is an important property expected from a simplicial theory. In particular, (53) implies that the simplicial action for a circle $S_{\Xi_{n}}$ given by (42) is compatible with simplicial aggregations $\Xi_{n} \rightarrow \Xi_{n^{\prime}}$ for $n, n^{\prime}$ odd.

\subsubsection{Quantum master equation.}

Lemma 4. ${ }^{11}$ The partition function for an interval (61) satisfies the following differential equation:

$$
\hbar \frac{\partial}{\partial \tilde{\psi}^{a}} \frac{\partial}{\partial A^{a}} Z_{\mathcal{I}}+\frac{1}{\hbar}\left[\frac{1}{6} f^{a b c} \hat{\psi}^{a} \hat{\psi}^{b} \hat{\psi}^{c}, Z_{\mathcal{I}}\right]_{C l(\mathfrak{g})}=0,
$$

where $[,]_{C l(\mathfrak{g})}$ denotes the super-commutator on $\mathrm{Cl}(\mathfrak{g})$.

Proof. We will check (68) using variables $(\lambda, A)$. We have,

$$
\left(\hbar \lambda^{a} \frac{\partial}{\partial A^{a}}+\frac{1}{\hbar}\left[\frac{1}{6} f^{a b c} \hat{\psi}^{a} \hat{\psi}^{b} \hat{\psi}^{c}, \bullet\right]_{C l(\mathfrak{g})}\right) \exp \left(-\frac{1}{2 \hbar} f^{a b c} \hat{\psi}^{a} A^{b} \hat{\psi}^{c}+\lambda^{a} \hat{\psi}^{a}\right)=0
$$

After the Fourier transform from the variable $\lambda$ to the variable $\tilde{\psi}$, this expression becomes (68)). Observe that

$$
\begin{aligned}
& \hbar \lambda^{a} \frac{\partial}{\partial A^{a}} \exp \left(-\frac{1}{2 \hbar} f^{a b c} \hat{\psi}^{a} A^{b} \hat{\psi}^{c}+\lambda^{a} \hat{\psi}^{a}\right) \\
& =\int_{0}^{1} d \tau e^{\tau\left(-\frac{1}{2 \hbar} f^{a b c} \hat{\psi}^{a} A^{b} \hat{\psi}^{c}+\lambda^{a} \hat{\psi}^{a}\right)} \cdot \frac{1}{2} f^{a b c} \hat{\psi}^{a} \lambda^{b} \hat{\psi}^{c} \cdot e^{(1-\tau)\left(-\frac{1}{2 \hbar} f^{a b c} \hat{\psi}^{a} A^{b} \hat{\psi}^{c}+\lambda^{a} \hat{\psi}^{a}\right)} .
\end{aligned}
$$

Next, compute commutators in the Clifford algebra,

$$
\left[\frac{1}{6} f^{a b c} \hat{\psi}^{a} \hat{\psi}^{b} \hat{\psi}^{c}, \lambda^{a^{\prime}} \hat{\psi}^{a^{\prime}}\right]_{C l(\mathfrak{g})}=-\frac{\hbar}{2} f^{a b c} \hat{\psi}^{a} \lambda^{b} \hat{\psi}^{c},
$$

and

$$
\begin{aligned}
& {\left[\frac{1}{6} f^{a b c} \hat{\psi}^{a} \hat{\psi}^{b} \hat{\psi}^{c}, \frac{1}{2} f^{a^{\prime} b^{\prime} c^{\prime}} \hat{\psi}^{a^{\prime}} A^{b^{\prime}} \hat{\psi}^{c^{\prime}}\right]_{C l(\mathfrak{g})}} \\
& =\frac{1}{12} f^{a b c} f^{a^{\prime} b^{\prime} c^{\prime}} A^{b^{\prime}}\left(\left[\hat{\psi}^{a} \hat{\psi}^{b} \hat{\psi}^{c}, \hat{\psi}^{a^{\prime}}\right] \hat{\psi}^{c^{\prime}}-\hat{\psi}^{a^{\prime}}\left[\hat{\psi}^{a} \hat{\psi}^{b} \hat{\psi}^{c}, \hat{\psi}^{c^{\prime}}\right]\right) \\
& =\frac{\hbar}{4} f^{a b c} f^{a^{\prime} b^{\prime} c^{\prime}} A^{b^{\prime}}\left(\delta^{c a^{\prime}} \hat{\psi}^{a} \hat{\psi}^{b} \hat{\psi}^{c^{\prime}}-\delta^{c c^{\prime}} \hat{\psi}^{a^{\prime}} \hat{\psi}^{a} \hat{\psi}^{b}\right) \\
& =\frac{\hbar}{4} f^{a b c} f^{c b^{\prime} c^{\prime}} A^{b^{\prime}}\left(\hat{\psi}^{a} \hat{\psi}^{b} \hat{\psi}^{c^{\prime}}+\hat{\psi}^{c^{\prime}} \hat{\psi}^{a} \hat{\psi}^{b}\right)
\end{aligned}
$$

11 In a different setting Eq. (68) appeared in [2]. 


$$
\begin{aligned}
= & \underbrace{\frac{\hbar}{6} f^{a b c} f^{c b^{\prime} c^{\prime}} A^{b^{\prime}}\left(\hat{\psi}^{a} \hat{\psi}^{b} \hat{\psi}^{c^{\prime}}+\hat{\psi}^{c^{\prime}} \hat{\psi}^{a} \hat{\psi}^{b}+\hat{\psi}^{b} \hat{\psi}^{c^{\prime}} \hat{\psi}^{a}\right)}_{=0 \text { by Jacobi identity }} \\
& +\frac{\hbar}{12} f^{a b c} f^{c b^{\prime} c^{\prime}} A^{b^{\prime}}\left(\hat{\psi}^{a} \hat{\psi}^{b} \hat{\psi}^{c^{\prime}}+\hat{\psi}^{c^{\prime}} \hat{\psi}^{a} \hat{\psi}^{b}-2 \hat{\psi}^{b} \hat{\psi}^{c^{\prime}} \hat{\psi}^{a}\right) \\
= & \frac{\hbar}{24} f^{a b c} f^{c b^{\prime} c^{\prime}} A^{b^{\prime}}\left(\left(\hat{\psi}^{a} \hat{\psi}^{b} \hat{\psi}^{c^{\prime}}+\hat{\psi}^{c^{\prime}} \hat{\psi}^{a} \hat{\psi}^{b}-2 \hat{\psi}^{b} \hat{\psi}^{c^{\prime}} \hat{\psi}^{a}\right)\right. \\
& \left.-\left(\hat{\psi}^{b} \hat{\psi}^{a} \hat{\psi}^{c^{\prime}}+\hat{\psi}^{c^{\prime}} \hat{\psi}^{b} \hat{\psi}^{a}-2 \hat{\psi}^{a} \hat{\psi}^{c^{\prime}} \hat{\psi}^{b}\right)\right) \\
= & \frac{\hbar}{24} f^{a b c} f^{c b^{\prime} c^{\prime}} A^{b^{\prime}}\left(\hat{\psi}^{a}\left[\hat{\psi}^{b}, \hat{\psi}^{c^{\prime}}\right]+\left[\hat{\psi}^{c^{\prime}}, \hat{\psi}^{a}\right] \hat{\psi}^{b}-\hat{\psi}^{b}\left[\hat{\psi}^{c^{\prime}}, \hat{\psi}^{a}\right]-\left[\hat{\psi}^{b}, \hat{\psi}^{c^{\prime}}\right] \hat{\psi}^{a}\right) \\
= & 0 .
\end{aligned}
$$

For brevity, we are omitting the subscript in $[,]_{C l(\mathfrak{g})}$ in computations. Identities $(71,72)$ imply

$$
\begin{aligned}
& \frac{1}{\hbar}\left[\frac{1}{6} f^{a b c} \hat{\psi}^{a} \hat{\psi}^{b} \hat{\psi}^{c}, \exp \left(-\frac{1}{2 \hbar} f^{a b c} \hat{\psi}^{a} A^{b} \hat{\psi}^{c}+\lambda^{a} \hat{\psi}^{a}\right)\right]_{C l(\mathfrak{g})} \\
& =-\int_{0}^{1} d \tau e^{\tau\left(-\frac{1}{2 \hbar} f^{a b c} \hat{\psi}^{a} A^{b} \hat{\psi}^{c}+\lambda^{a} \hat{\psi}^{a}\right)} \cdot \frac{1}{2} f^{a b c} \hat{\psi}^{a} \lambda^{b} \hat{\psi}^{c} \cdot e^{(1-\tau)\left(-\frac{1}{2 \hbar} f^{a b c} \hat{\psi}^{a} A^{b} \hat{\psi}^{c}+\lambda^{a} \hat{\psi}^{a}\right)} .
\end{aligned}
$$

Together with (70), this implies (69) which finishes the proof of (68).

Let us denote ${ }^{12}$ by

$$
\hat{\theta}:=\frac{1}{6} f^{a b c} \hat{\psi}^{a} \hat{\psi}^{b} \hat{\psi}^{c}
$$

the Clifford element in (68).

Theorem 3. The partition function for any one-dimensional simplicial complex $\Theta$ satisfies the differential equation

$$
\left(\hbar \Delta_{\Theta}^{\text {bulk }}+\frac{1}{\hbar} \delta_{\Theta}\right) Z_{\Theta}=0
$$

where

$$
\Delta_{\Theta}^{\mathrm{bulk}}=\sum_{k} \frac{\partial}{\partial \tilde{\psi}_{k}^{a}} \frac{\partial}{\partial A_{k}^{a}}
$$

(the sum goes over all 1-simplices of $\Theta$ ), and

$$
\delta_{\Theta}=\sum_{j=1}^{i(\Theta)}\left[\hat{\theta}^{(j)}, \bullet\right]_{C l(\mathfrak{g})} .
$$

Here the sum goes over connected components of $\Theta$ that are triangulated intervals; $\hat{\theta}^{(j)}$ denotes $\hat{\theta}$ as an element of the $j^{\text {th }}$ copy of $C l(\mathfrak{g})$.

\footnotetext{
12 The notation stems from the fact that this is a quantization of the Maurer-Cartan element $\theta \in \operatorname{Fun}(\Pi \mathfrak{g})$ (4).
} 
Proof. Equation (73) follows from (68). The compatibility with disjoint unions is obvious as $\Delta_{\Theta_{1} \sqcup \Theta_{2}}^{\text {bulk }}=\Delta_{\Theta_{1}}^{\text {bulk }}+\Delta_{\Theta_{2}}^{\text {bulk }}$, and $\delta_{\Theta_{1}} \sqcup \Theta_{2}=\delta_{\Theta_{1}}+\delta_{\Theta_{2}}$. For concatenations, it suffices to check the case of two triangulated intervals $\Theta_{1}$ and $\Theta_{2}$ :

$$
\begin{aligned}
\left(\hbar \Delta_{\Theta_{1} \cup \Theta_{2}}^{\text {bulk }}+\hbar^{-1} \delta_{\Theta_{1} \cup \Theta_{2}}\right) Z_{\Theta_{1} \cup \Theta_{2}=} & \left(\hbar \Delta_{\Theta_{1}}^{\text {bulk }}+\hbar \Delta_{\Theta_{2}}^{\text {bulk }}+\hbar^{-1}[\hat{\theta}, \bullet]_{C l(\mathfrak{g})}\right) \circ\left(Z_{\Theta_{2}} \cdot Z_{\Theta_{1}}\right) \\
= & Z_{\Theta_{2}} \cdot\left(\left(\hbar \Delta_{\Theta_{1}}^{\text {bulk }}+\hbar^{-1}[\hat{\theta}, \bullet]_{C l(\mathfrak{g})}\right) \circ Z_{\Theta_{1}}\right) \\
& +\left(\left(\hbar \Delta_{\Theta_{2}}^{\text {bulk }}+\hbar^{-1}[\hat{\theta}, \bullet]_{C l(\mathfrak{g})}\right) \circ Z_{\Theta_{2}}\right) \cdot Z_{\Theta_{1}} \\
= & 0 .
\end{aligned}
$$

The compatibility with closure of a triangulated interval into a triangulated circle follows from $\operatorname{Str}_{C l(\mathfrak{g})}\left[\hat{\theta}, Z_{\Theta}\right]_{C l(\mathfrak{g})}=0$.

Remark 20. We understand (73) as a kind of quantum master equation with the boundary term $\delta_{\Theta} Z_{\Theta}$. It is tempting to think of the operator $\Delta_{\Theta}^{\text {bulk }}+\delta_{\Theta}$ appearing in (73) as a new BV Laplacian adjusted for the presence of the boundary.

Corollary 1. In the case of a triangulated circle $\Theta=\Xi_{n}$, the partition function satisfies the usual (non-modified) quantum master equation

$$
\Delta_{\Xi_{n}} Z_{\Xi_{n}}=0 .
$$

\section{Back to Path Integral}

Sections 4.1 and 4.4 mostly go along the lines of the standard derivation of the path integral representation for quantum mechanics, see [9].

4.1. Representation of $C l(\mathfrak{g})$, complex polarization of $\mathfrak{g}$. The Clifford algebra $\mathrm{Cl}(\mathfrak{g})$ admits a representation $\rho$ on the space of polynomials of $m$ odd variables Fun $\left(\mathbb{C}^{0 \mid m}\right) \cong$ $\mathbb{C}\left[\eta^{1}, \ldots, \eta^{m}\right]$. This representation is defined on generators of $C l(\mathfrak{g})$ as

$$
\rho: \hat{\psi}^{a} \mapsto \begin{cases}\frac{1}{\sqrt{2}}\left(\eta^{p}+\hbar \frac{\partial}{\partial \eta^{p}}\right) & \text { if } a=2 p-1, \\ \frac{i}{\sqrt{2}}\left(\eta^{p}-\hbar \frac{\partial}{\partial \eta^{p}}\right) & \text { if } a=2 p .\end{cases}
$$

In fact, $\rho: C l(\mathfrak{g}) \rightarrow \operatorname{End}\left(\operatorname{Fun}\left(\mathbb{C}^{0 \mid m}\right)\right) \cong \operatorname{End}\left(\mathbb{C}^{2^{m-1} \mid 2^{m-1}}\right)$ is an isomorphism of superalgebras. There is a natural identification

$$
\phi: \quad \operatorname{End}\left(\operatorname{Fun}\left(\mathbb{C}^{0 \mid m}\right)\right) \stackrel{\sim}{\rightarrow} \operatorname{Fun}\left(\mathbb{C}^{0 \mid m} \oplus \mathbb{C}^{0 \mid m}\right) \cong \mathbb{C}\left[\eta^{1}, \ldots, \eta^{m}, \bar{\eta}^{1}, \ldots, \bar{\eta}^{m}\right] .
$$

This is not an algebra morphism with respect to the standard algebra structure on polynomials. Instead, it takes the product of endomorphisms into the convolution; see [3,9]). We are interested in the composition $\Phi=\phi \circ \rho: C l(\mathfrak{g}) \rightarrow \operatorname{Fun}\left(\mathbb{C}^{0 \mid m} \oplus \mathbb{C}^{0 \mid m}\right)$ which maps

$$
\Phi: \quad\left\{\begin{array}{l}
\hat{1} \mapsto e^{\frac{1}{\hbar} \sum_{q} \eta^{q} \bar{\eta}^{q}} \\
\hat{\psi}^{2 p-1} \mapsto \frac{1}{\sqrt{2}}\left(\eta^{p}+\bar{\eta}^{p}\right) e^{\frac{1}{\hbar} \sum_{q} \eta^{q} \bar{\eta}^{q}} \\
\hat{\psi}^{2 p} \mapsto \frac{i}{\sqrt{2}}\left(\eta^{p}-\bar{\eta}^{p}\right) e^{\frac{1}{\hbar} \sum_{q} \eta^{q} \bar{\eta}^{q}}
\end{array}\right.
$$


and sends the product in $C l(\mathfrak{g})$ into the convolution

$$
\begin{aligned}
& \Phi(\hat{\alpha} \cdot \hat{\beta})\left(\eta_{2}, \bar{\eta}_{1}\right) \\
& \quad=\hbar^{m} \int \prod_{p}\left(D \eta_{1}^{p} D \bar{\eta}_{2}^{p}\right) \Phi(\hat{\alpha})\left(\eta_{2}, \bar{\eta}_{2}\right) \cdot e^{\frac{1}{\hbar} \sum_{q} \bar{\eta}_{2}^{q} \eta_{1}^{q}} \cdot \Phi(\hat{\beta})\left(\eta_{1}, \bar{\eta}_{1}\right) .
\end{aligned}
$$

Formula (76) is a key point in reconstructing the path integral from the operator formalism. Another useful identity is as follows:

$$
\operatorname{Str}_{C l(\mathfrak{g})}(\hat{\alpha})=\hbar^{m} \int \prod_{p}\left(D \eta^{p} D \bar{\eta}^{p}\right) e^{\frac{1}{\hbar} \sum_{q} \bar{\eta}^{q} \eta^{q}} \cdot \Phi(\hat{\alpha})(\eta, \bar{\eta}) .
$$

More generally, a representation of type (74) is associated to a choice of a linear complex structure $J$ on $\mathfrak{g}$ compatible with the pairing:

$$
J: \mathfrak{g} \rightarrow \mathfrak{g}, \quad J^{2}=-\mathrm{id}_{\mathfrak{g}}, \quad(J a, b)=-(a, J b) \quad \text { for } a, b \in \mathfrak{g} .
$$

It induces the splitting of the complexified Lie algebra $\mathfrak{g}_{\mathbb{C}}=\mathbb{C} \otimes \mathfrak{g}$ into "holomorphic" and "anti-holomorphic" subspaces:

$$
\mathfrak{g} \mathbb{C}=\mathfrak{h} \oplus \overline{\mathfrak{h}},
$$

where $J$ acts on $\mathfrak{h}, \overline{\mathfrak{h}}$ by multiplication by $+i$ and $-i$, respectively. (Note that $\mathfrak{h}$ and $\overline{\mathfrak{h}}$ are complex subspaces of $\mathfrak{g}_{\mathbb{C}}$ with respect to the standard complex structure; bar in $\overline{\mathfrak{h}}$ does not mean conjugation.) The subspaces $\mathfrak{h}$ are $\overline{\mathfrak{h}}$ are Lagrangian with respect to the pairing (, ). The complex Lagrangian polarization (78) induces a polarization for the parity-reversed Lie algebra

$$
\Pi \mathfrak{g}=\Pi \mathfrak{h} \oplus \Pi \overline{\mathfrak{h}} .
$$

We denote coordinates on $\Pi \mathfrak{h}$ by $\eta^{1}, \ldots, \eta^{m}$ and coordinates on $\Pi \overline{\mathfrak{h}}$ by $\bar{\eta}^{1}, \ldots, \bar{\eta}^{m}$. The representation $\rho: C l(\mathfrak{g})=\widehat{\operatorname{Fun}(\Pi \mathfrak{g})} \rightarrow \operatorname{End}(\operatorname{Fun}(\Pi \mathfrak{h}))$ sends quantized holomorphic coordinates to multiplication operators and quantized anti-holomorphic coordinates to partial derivatives:

$$
\rho:\left\{\begin{array}{l}
\hat{\eta}^{p} \mapsto \eta^{p} \cdot \\
\hat{\bar{\eta}}^{p} \mapsto \hbar \frac{\partial}{\partial \eta^{p}}
\end{array}\right.
$$

Morphism (75) from $C l(\mathfrak{g})$ to the convolution algebra $\operatorname{Fun}(\Pi \mathfrak{h} \oplus \Pi \overline{\mathfrak{h}})$ is given on generators by

$$
\Phi:\left\{\begin{array}{l}
\hat{\eta}^{p} \mapsto \eta^{p} e^{\frac{1}{\hbar} \sum_{q} \eta^{q} \bar{\eta}^{q}} \\
\hat{\bar{\eta}}^{p} \mapsto \bar{\eta}^{p} e^{\frac{1}{\hbar} \sum_{q} \eta^{q} \bar{\eta}^{q}}
\end{array}\right.
$$

and it extends to the other elements of $C l(\mathfrak{g})$ by the convolution formula (76).

We will use notation $\pi, \bar{\pi}$ for projections from $\Pi \mathfrak{g}$ to $\Pi \mathfrak{h}$ and $\Pi \overline{\mathfrak{h}}$, respectively. We denote by $\iota, \bar{\imath}$ embeddings of $\Pi \mathfrak{h}$ and $\Pi \overline{\mathfrak{h}}$ into $\Pi \mathfrak{g}$. 
4.2. One-dimensional Chern-Simons in terms of Atiyah-Segal's axioms. To a point with positive orientation we associate the vector super-space (the space of states)

$$
\mathcal{H}_{p t^{+}}=\operatorname{Fun}(\Pi \mathfrak{h}) \cong \mathbb{C}\left[\eta^{1}, \ldots, \eta^{m}\right],
$$

and to a point with negative orientation - the dual space

$$
\mathcal{H}_{p t^{-}}=\left(\mathcal{H}_{p t^{+}}\right)^{*}=\operatorname{Fun}(\Pi \overline{\mathfrak{h}}) \cong \mathbb{C}\left[\bar{\eta}^{1}, \ldots, \bar{\eta}^{m}\right] .
$$

To an interval $\mathcal{I}=\left[\mathfrak{p}_{1}, \mathfrak{p}_{2}\right]$ we associate the partition function

$$
Z_{\mathcal{I}}^{\rho}:=\rho\left(Z_{\mathcal{I}}\right) \in \operatorname{Fun}(\Pi \mathfrak{g} \oplus \mathfrak{g}) \otimes \underbrace{\mathcal{H}_{\mathfrak{p}_{2}^{+}} \otimes \mathcal{H}_{\mathfrak{p}_{1}^{-}}}_{\cong \operatorname{End}\left(\mathcal{H}_{p t^{+}}\right)}
$$

given by formula (61) in representation $\rho$ (see Eq. (74)). In general, to a one-dimensional simplicial complex $\Theta$ we associate the partition function (60) of Sect. 3.2.1 taken in representation $\rho$ :

$$
Z_{\Theta}^{\rho}:=\rho^{\otimes i(\Theta)} \circ Z_{\Theta} \in \operatorname{Fun}\left(\mathcal{F}_{\Theta}^{\text {bulk }}\right) \otimes\left(\mathcal{H}_{p t^{+}} \otimes \mathcal{H}_{p t^{-}}\right)^{\otimes i(\Theta)} .
$$

The one-dimensional Chern-Simons theory features three types of operations:

- To a disjoint union $\Theta_{1} \sqcup \Theta_{2}$ corresponds the tensor product for partition functions.

- To a sewing of boundary points $\mathfrak{p}_{1}^{-}$and $\mathfrak{p}_{2}^{+}$in a simplicial complex $\Theta$ corresponds the convolution of spaces of states $\mathcal{H}_{\mathfrak{p}_{2}^{+}}$and $\mathcal{H}_{\mathfrak{p}_{1}^{-}}$.

- To a simplicial aggregation $r: \Theta \rightarrow \Theta^{\prime}$ corresponds a fiber BV integral $r_{*}$ which reduces the space of bulk fields from $\mathcal{F}_{\Theta}^{\text {bulk }}$ to $\mathcal{F}_{\Theta^{\prime}}^{\text {bulk }}$.

In addition, $\mathcal{H}_{p t^{+}}$is equipped with an odd third-order differential operator

$$
\delta^{\rho}=\rho(\hat{\theta}): \mathcal{H}_{p t^{+}} \rightarrow \mathcal{H}_{p t^{+}},
$$

and $\mathcal{H}_{p t^{-}}$is equipped with minus its dual $-\left(\delta^{\rho}\right)^{*}: \mathcal{H}_{p t^{-}} \rightarrow \mathcal{H}_{p t^{-}}$. The partition function $Z_{\Theta}^{\rho}$ satisfies the quantum master equation

$$
\left(\hbar \Delta_{\Theta}^{\text {bulk }}+\hbar^{-1} \delta_{\Theta}^{\rho}\right) Z_{\Theta}^{\rho}=0,
$$

where the "boundary BV operator" $\delta_{\Theta}^{\rho}$ is the sum over boundary points of $\Theta$ of operators $\delta^{\rho}$ or $-\left(\delta^{\rho}\right)^{*}$ acting on the corresponding $\mathcal{H}_{p t}$ (depending on whether the orientation of $p t$ is positive or negative).

Remark 21. $\delta^{\rho}$ is "almost" a coboundary operator: its square is proportional to identity:

$$
\left(\delta^{\rho}\right)^{2}=-\frac{\hbar^{3}}{48} f^{a b c} f^{a b c} \cdot \mathrm{id}_{\mathcal{H}_{p t^{+}}}
$$

(see [12]). This implies that the boundary BV operator for an interval

$$
\delta_{\mathcal{I}}^{\rho}=\delta^{\rho} \otimes \operatorname{id}_{\mathcal{H}_{p t^{-}}}-\mathrm{id}_{\mathcal{H}_{p t^{+}}} \otimes\left(\delta^{\rho}\right)^{*}: \underbrace{\mathcal{H}_{p t^{+}} \otimes \mathcal{H}_{p t^{-}}}_{\cong \operatorname{End}\left(\mathcal{H}_{p t^{+}}\right)} \rightarrow \underbrace{\mathcal{H}_{p t^{+}} \otimes \mathcal{H}_{p t^{-}}}_{\cong \operatorname{End}\left(\mathcal{H}_{p t^{+}}\right)}
$$

squares to zero

$$
\left(\delta_{\mathcal{I}}^{\rho}\right)^{2}=0
$$

Cases when $\delta^{\rho}$ squares to zero (i.e. when $f^{a b c} f^{a b c}=0$ ) are quite interesting as then the reduced space of states for a point $\mathcal{H}_{p t^{+}}^{\text {red }}$ emerges (see Remarks 25, 34, 35 below). 
Remark 22. The space of states $\mathcal{H}_{p t^{+}}$can be viewed as a geometric quantization of the classical phase super-space $\Pi \mathfrak{g}$ (viewed as an odd Kähler manifold). The operator $\delta^{\rho}$ is the quantization of the Maurer-Cartan element $\theta$ (4); the operator $\hbar^{-1} \delta_{\mathcal{I}}^{\rho}$ is the quantization of the Hamiltonian vector field $\{\theta, \bullet\}$ on $\Pi \mathfrak{g}$.

Remark 23. Topological quantum mechanics (TQM) in the sense of A. Losev [13] assigns to an interval a manifold Geom (the "space of geometric data") and to a point - a vector superspace $\mathcal{H}$ endowed with an odd coboundary operator $Q$. The evolution operator $U$ for an interval is a differential form on $\operatorname{Geom}$ with values in $\operatorname{End}(\mathcal{H})$ and has to satisfy the "homotopy topologicity" equation

$$
\left(d+\operatorname{ad}_{Q}\right) U=0
$$

where $d$ is the de Rham operator on Geom. A standard class of examples of TQMs comes from choosing Geom $=\mathbb{R}_{>0}$ (with coordinate $t>0$ ) and setting

$$
U(t, d t)=e^{[Q, G] t+d t G}=e^{\left(d+\mathrm{ad}_{Q}\right) \circ(t G)},
$$

where $G$ is an odd operator on $\mathcal{H}$. For instance, for the Hodge TQM [13] on a Riemannian manifold $M$, one sets $\mathcal{H}=\Omega^{\bullet}(M), Q=d_{M}$ and $G=d_{M}^{*}$ - the Hodge operator on forms on $M$. For the Morse TQM [10,18], one takes the same $\mathcal{H}$ and $Q$, but now $G=\iota_{v}$ is the substitution of the gradient vector field. The one-dimensional Chern-Simons theory on an interval can be viewed as a TQM: here Geom $=\mathfrak{g}$ (with coordinates $A^{a}$ ), $\mathcal{H}=\operatorname{Fun}(\Pi \mathfrak{h}), Q=\hbar^{-1} \delta^{\rho}$. The odd Fourier transform in variable $\tilde{\psi}$ of the partition function for an interval (61) is

$$
U(A, \lambda)=\rho\left(e^{-\frac{1}{2 \hbar} f^{a b c} \hat{\psi}^{a} A^{b} \hat{\psi}^{c}+\lambda^{a} \hat{\psi}^{a}}\right)=e^{\left(d+\hbar^{-1} \operatorname{ad}_{\delta} \rho\right) \circ \hbar^{-1} \rho\left(A^{a} \hat{\psi}^{a}\right)},
$$

where $d=\hbar \lambda^{a} \frac{\partial}{\partial A^{a}}$ is the de Rham operator on Geom. Note that the expression (86) is similar to (85) where we make a substitution $t G \mapsto \hbar^{-1} \rho\left(A^{a} \hat{\psi}^{a}\right)$. The quantum master equation (68) is equivalent to

$$
\left(d+\hbar^{-1} \operatorname{ad}_{\delta^{\rho}}\right) U(A, \lambda)=0
$$

which is exactly the "homotopy topologicity" equation (84). The peculiarity of the onedimensional Chern-Simons theory viewed as a TQM is that $\delta^{\rho}$ is not necessarily a coboundary operator on $\mathcal{H}$.

4.3. Integrating out the bulk fields. In Sect. 3.2.2, we discussed simplicial aggregations which reduce the space of bulk fields of the 1-dimensional Chern-Simons theory $\mathcal{F}_{\Theta}^{\text {bulk }} \rightarrow \mathcal{F}_{\Theta^{\prime}}^{\text {bulk }}$ according to combinatorial moves applied to the triangulation $\Theta \rightarrow \Theta^{\prime}$. It is interesting to consider the "ultimate aggregation" — integrating out the bulk fields completely. This procedure should yield the partition function in the sense of AtiyahSegal (i.e. without bulk fields). We will denote it by $Z^{\circ}$.

For an interval, we have

$$
(i \hbar)^{m} \int D \tilde{\psi} Z_{\mathcal{I}}(\tilde{\psi}, A)=e^{-\frac{1}{2 \hbar} f^{a b c} \hat{\psi}^{a} A^{b} \hat{\psi}^{c}}
$$


We view (88) as a BV integral over the Lagrangian subspace

$$
\mathcal{L}_{A}=\{\tilde{\psi}+A \mid \tilde{\psi} \text { is free, } A \text { fixed }\} \subset \mathcal{F}_{\mathcal{I}}^{\text {bulk }} .
$$

This subspace depends on the value of $A$, and integral (88) also depends on $A$. However, this dependence is $\operatorname{ad}_{\hat{\theta}}$-exact:

$$
\begin{aligned}
& e^{-\frac{1}{2 \hbar} f^{a b c} \hat{\psi}^{a}(A+\delta A)^{b} \hat{\psi}^{c}}-e^{-\frac{1}{2 \hbar} f^{a b c} \hat{\psi}^{a} A^{b} \hat{\psi}^{c}} \\
& \quad=\frac{1}{\hbar}\left[\hat{\theta}, e^{-\frac{1}{2 \hbar} f^{a b c} \hat{\psi}^{a} A^{b} \hat{\psi}^{c}+\frac{1}{\hbar} \delta A^{a} \hat{\psi}^{a}}\right]_{C l(\mathfrak{g})}+\mathcal{O}\left((\delta A)^{2}\right)
\end{aligned}
$$

(this can be checked analogously to the proof of Lemma 4). Therefore, we should understand the partition function $Z_{\mathcal{I}}^{\circ}$ as an element of cohomology of the operator $\operatorname{ad}_{\hat{\theta}}$ (the fact that (88) is $\operatorname{ad}_{\hat{\theta}}$-closed is an immediate consequence of the quantum master equation (68)). More exactly, $Z_{\mathcal{I}}^{\circ}$ is the class of Clifford unit $\hat{1}$ in $\operatorname{ad}_{\hat{\theta}}$-cohomology:

$$
Z_{\mathcal{I}}^{\circ}=[\hat{1}] \in H_{\mathrm{ad}_{\hat{\theta}}}(C l(\mathfrak{g})) .
$$

Equivalently, in terms of representation $\rho$, we have

$$
\rho\left(Z_{\mathcal{I}}^{\circ}\right)=\left[\operatorname{id}_{\mathcal{H}_{p t^{+}}}\right] \in H_{\delta_{\mathcal{I}}^{\rho}}\left(\operatorname{End}\left(\mathcal{H}_{p t^{+}}\right)\right)
$$

Remark 24. If the contraction of structure constants $f^{a b c} f^{a b c}$ for $\mathfrak{g}$ is nonzero, the cohomology class (91), (92) vanishes since

$$
\hat{1}=-\frac{24 \hbar^{-3}}{f^{a b c} f^{a b c}} \operatorname{ad}_{\hat{\theta}} \hat{\theta} .
$$

In fact, the whole cohomology group $H_{\mathrm{ad}} \cong H_{\delta_{\mathcal{I}}^{\rho}}$ vanishes since every $\operatorname{ad}_{\hat{\theta}}$-cocycle $\hat{\alpha} \in C l(\mathfrak{g})$ is automatically exact:

$$
\hat{\alpha}=-\frac{24 \hbar^{-3}}{f^{a b c} f^{a b c}} \operatorname{ad}_{\hat{\theta}}(\hat{\theta} \cdot \hat{\alpha}) .
$$

Remark 25. If $f^{a b c} f^{a b c}=0$, we can define the reduced space of states for a point as the $\delta^{\rho}$-cohomology:

$$
\mathcal{H}_{p t^{+}}^{\mathrm{red}}:=H_{\delta^{\rho}}\left(\mathcal{H}_{p t^{+}}\right), \quad \mathcal{H}_{p t^{-}}^{\mathrm{red}}:=H_{-\left(\delta^{\rho}\right)^{*}}\left(\mathcal{H}_{p t^{-}}\right)=\left(\mathcal{H}_{p t^{+}}^{\mathrm{red}}\right)^{*} .
$$

By the Künneth formula, we have

$$
H_{\delta_{\mathcal{I}}^{\rho}}\left(\operatorname{End}\left(\mathcal{H}_{p t^{+}}\right)\right) \cong \mathcal{H}_{p t^{+}}^{\mathrm{red}} \otimes \mathcal{H}_{p t^{-}}^{\mathrm{red}} .
$$

The partition function (92) is then represented by the identity operator

$$
\rho\left(Z_{\mathcal{I}}^{\circ}\right): \quad \mathcal{H}_{p t^{+}}^{\text {red }} \stackrel{\text { id }}{\rightarrow} \mathcal{H}_{p t^{+}}^{\text {red }}
$$

For a circle, we can obtain the partition function $Z_{\mathcal{S}^{1}}^{\circ}$ (which is just a number) either as a Clifford super-trace of (88) or as a BV integral of the effective action (13) over the Lagrangian subspace (89). Either way, we have

$$
Z_{\mathcal{S}^{1}}^{\circ}=0,
$$

due to non-saturation of fermionic modes either in the Clifford super-trace or in the Berezin integral over $\tilde{\psi}$. 


\subsection{From operator formalism to path integral.}

4.4.1. Abelian one-dimensional Chern-Simons theory. The one-dimensional abelian Chern-Simons theory associates to an interval $\mathcal{I}$ the unit of $C l(\mathfrak{g})$ (here $\mathfrak{g}$ can be viewed as a Euclidean vector space; the Lie algebra structure is irrelevant). The path integral arises upon applying the map (75) to this trivial partition function:

$$
\begin{aligned}
\Phi(\hat{1})\left(\eta_{\text {out }}, \bar{\eta}_{\text {in }}\right)= & \Phi(\underbrace{\hat{1} \cdot \hat{1} \cdots \hat{1}}_{N})\left(\eta_{\text {out }}, \bar{\eta}_{\text {in }}\right) \\
= & \int\left(\prod_{k=1}^{N-1} \hbar^{m} D \eta_{k} D \bar{\eta}_{k+1}\right) . \\
& \cdot \exp \frac{1}{\hbar}\left(\left\langle\eta_{\text {out }}, \bar{\eta}_{N}\right\rangle+\left\langle\bar{\eta}_{N}, \eta_{N-1}\right\rangle+\left\langle\eta_{N-1}, \bar{\eta}_{N-1}\right\rangle\right. \\
& \left.+\cdots+\left\langle\eta_{2}, \bar{\eta}_{2}\right\rangle+\left\langle\bar{\eta}_{2}, \eta_{1}\right\rangle+\left\langle\eta_{1}, \bar{\eta}_{i n}\right\rangle\right) \\
= & \int\left(\prod_{k=1}^{N-1} \hbar^{m} D \eta_{k} D \bar{\eta}_{k+1}\right) \cdot \exp \frac{1}{\hbar}\left(\left\langle\eta_{1}, \bar{\eta}_{i n}\right\rangle+\sum_{k=2}^{N}\left\langle\eta_{k}-\eta_{k-1}, \bar{\eta}_{k}\right\rangle\right) .
\end{aligned}
$$

For convenience, we set $\bar{\eta}_{1}:=\bar{\eta}_{i n}, \eta_{N}:=\eta_{o u t}$ and introduced a notation

$$
\langle\eta, \bar{\eta}\rangle:=\sum_{q} \eta^{q} \bar{\eta}^{q}
$$

In the exponential of (95), $N$ terms of type $\left\langle\eta_{k}, \bar{\eta}_{k}\right\rangle$ correspond to Clifford units, and $N-1$ terms of type $\left\langle\bar{\eta}_{k+1}, \eta_{k}\right\rangle$ correspond to convolutions kernels as in (76); the symbol $D \eta_{k} D \bar{\eta}_{k+1}$ is defined as $\prod_{p}\left(D \eta_{k}^{p} D \bar{\eta}_{k+1}^{p}\right)$. Expression (95) corresponds to triangulating an interval by $N$ smaller intervals; the terms in the exponential correspond to 0 - and 1 -simplices of this triangulation. In the limit $N \rightarrow \infty$, one formally writes (95) as a path integral over paths $\eta(\tau), \bar{\eta}(\tau)$ with $\eta$ at the right end-point and $\bar{\eta}$ at left end-point of $\mathcal{I}$ fixed by the boundary conditions.

\section{Lemma 5.}

$$
\Phi(\hat{1})\left(\eta_{\text {out }}, \bar{\eta}_{\text {in }}\right)=\int_{\bar{\eta}(0)=\bar{\eta}_{\text {in }}, \eta(1)=\eta_{\text {out }}} \mathcal{D} \eta \mathcal{D} \bar{\eta} \cdot \exp \frac{1}{\hbar}\left(\langle\eta(0), \bar{\eta}(0)\rangle+\int_{\mathcal{I}}\langle d \eta, \bar{\eta}\rangle\right) .
$$

A perturbative computation of the path integral (96) is trivial: the integral is given by the contribution of the critical point

$$
\eta(\tau)=\eta_{\text {out }}, \quad \bar{\eta}(\tau)=\bar{\eta}_{\text {in }} \text { for all } \tau \in[0,1]
$$

and yields

$$
\Phi(\hat{1})\left(\eta_{\text {out }}, \bar{\eta}_{\text {in }}\right)=e^{\frac{1}{\hbar}\left\langle\eta_{\text {out }}, \bar{\eta}_{\text {in }}\right\rangle} .
$$

It is instructive to write the integral (96) in terms of the field $\psi$ instead of fields $\eta, \bar{\eta}$. For simplicity, we first choose a complex polarization (as in (74))

$$
\left\{\begin{array} { r l } 
{ \psi ^ { 2 p - 1 } } & { = \frac { 1 } { \sqrt { 2 } } ( \eta ^ { p } + \overline { \eta } ^ { p } ) } \\
{ \psi ^ { 2 p } } & { = \frac { i } { \sqrt { 2 } } ( \eta ^ { p } - \overline { \eta } ^ { p } ) }
\end{array} \Leftrightarrow \left\{\begin{array}{rl}
\eta^{p} & =\frac{1}{\sqrt{2}}\left(\psi^{2 p-1}-i \psi^{2 p}\right) \\
\bar{\eta}^{p} & =\frac{1}{\sqrt{2}}\left(\psi^{2 p-1}+i \psi^{2 p}\right)
\end{array}\right.\right.
$$


(this corresponds to the complex structure $J$ on $\mathfrak{g}$ which assigns $\psi^{2 p-1}$ as "real" coordinates and $\psi^{2 p}$ as "imaginary" coordinates on $\left.\Pi \mathfrak{g}\right)$. We have,

$$
\begin{aligned}
\sum_{p} \eta_{k}^{p} \bar{\eta}_{k}^{p} & =\sum_{p} i \psi_{k}^{2 p-1} \psi_{k}^{2 p} \\
\sum_{p} \bar{\eta}_{k+1}^{p} \eta_{k}^{p} & =\sum_{p} \frac{\psi_{k+1}^{2 p-1} \psi_{k}^{2 p-1}+\psi_{k+1}^{2 p} \psi_{k}^{2 p}}{2}-\sum_{p} i \frac{\psi_{k+1}^{2 p-1} \psi_{k}^{2 p}-\psi_{k+1}^{2 p} \psi_{k}^{2 p-1}}{2}
\end{aligned}
$$

Substituting these expressions into the integral representation (95) for $\Phi(1)$, we obtain

$$
\begin{aligned}
& \Phi(\hat{1})\left(\eta_{\text {out }}, \bar{\eta}_{\text {in }}\right)=\int\left(\hbar^{m / 2} D \eta_{1}\right)\left(\prod_{k=2}^{N-1}(i \hbar)^{m} D \psi_{k}\right)\left(\hbar^{m / 2} D \bar{\eta}_{N}\right) \\
& \quad \cdot \exp \frac{1}{\hbar}\left(\sum_{k=1}^{N-1} \frac{1}{2}\left(\psi_{k+1}, \psi_{k}\right)+\sum_{p} \sum_{k=1}^{N-1} \frac{i}{2}\left(\psi_{k+1}^{2 p-1}-\psi_{k}^{2 p-1}\right)\left(\psi_{k+1}^{2 p}-\psi_{k}^{2 p}\right)\right. \\
& \left.\quad+\sum_{p} \frac{i}{2} \psi_{1}^{2 p-1} \psi_{1}^{2 p}+\sum_{p} \frac{i}{2} \psi_{N}^{2 p-1} \psi_{N}^{2 p}\right) .
\end{aligned}
$$

Here $D \psi_{k}:=\coprod_{a} D \psi_{k}^{a}$ is the Berezin measure on $\Pi \mathfrak{g}$; the variable $\psi_{1}$ is constructed by formulae (97) from the integration variable $\eta_{1}$ and the boundary value $\bar{\eta}_{1}:=\bar{\eta}_{i n}$, and $\psi_{N}$ is constructed from the integration variable $\bar{\eta}_{N}$ and the boundary value $\eta_{N}:=\eta_{\text {out }}$.

Remark 26. We think of the integral (95) as corresponding to cutting the interval $\mathcal{I}=\left[\mathfrak{p}_{\text {in }}, \mathfrak{p}_{\text {out }}\right]$ into $N$ intervals $\left[\mathfrak{p}_{\text {in }}, \mathfrak{p}_{2}\right] \cup\left[\mathfrak{p}_{2}, \mathfrak{p}_{3}\right] \cup \cdots \cup\left[\mathfrak{p}_{N}, \mathfrak{p}_{\text {out }}\right]$. Integration variables $\eta_{k}, \bar{\eta}_{k+1}$ are associated to the point $\mathfrak{p}_{k+1}$ (more specifically, to the right end of the interval $\left[\mathfrak{p}_{k}, \mathfrak{p}_{k+1}\right]$ and to the left end of the interval $\left[\mathfrak{p}_{k+1}, \mathfrak{p}_{k+2}\right]$, respectively); the boundary value $\bar{\eta}_{i n}$ corresponds to the point $\mathfrak{p}_{i n}$, the boundary value $\eta_{\text {out }}$ — to the point $\mathfrak{p}_{\text {out }}$. However, variables $\psi_{k}$ are linear combinations of $\eta_{k}$ and $\bar{\eta}_{k}$. Thus, they are not associated to any single point, but rather to a pair of neighboring points $\left(\mathfrak{p}_{k}, \mathfrak{p}_{k+1}\right)$.

Again, we formally write the limit $N \rightarrow \infty$ of the integral (98) as a path integral over paths $\psi: \mathcal{I} \rightarrow \Pi \mathfrak{g}$ with a fixed anti-holomorphic projection of $\psi$ at the right end-point of the interval and a fixed holomorphic projection at the left end-point:

Lemma 6. The path integral expression for the partition function of the abelian ChernSimons theory on an interval is given by

$$
\begin{aligned}
\Phi(\hat{1})\left(\eta_{\text {out }}, \bar{\eta}_{\text {in }}\right)= & \int_{\bar{\pi}(\psi(0))=\bar{\eta}_{\text {in }}, \pi(\psi(1))=\eta_{\text {out }}} \mathcal{D} \psi \\
& \cdot \exp \frac{1}{\hbar}\left(\sum_{p} \frac{i}{2} \psi^{2 p-1}(0) \psi^{2 p}(0)+\int_{\mathcal{I}} \frac{1}{2}(\psi, d \psi)\right. \\
& \left.+\sum_{p} \frac{i}{2} \psi^{2 p-1}(1) \psi^{2 p}(1)\right)
\end{aligned}
$$


The exact meaning of the conditional measure on paths in (99) is the formal $N \rightarrow \infty$ limit of the measure in (98). The second term in the exponential in (98) does not contribute to the limit $N \rightarrow \infty$ : once we assume that $\psi_{k}$ are values of a differentiable path $\psi(\tau)$ at times $\tau=k / N$, the contribution of this term becomes of order $\mathcal{O}(1 / N)$.

For a general complex structure $J$ on $\mathfrak{g}$, path integral (99) becomes

$$
\begin{aligned}
\Phi(\hat{1})\left(\eta_{\text {out }}, \bar{\eta}_{\text {in }}\right)= & \int_{\bar{\pi}(\psi(0))=\bar{\eta}_{\text {in }}, \pi(\psi(1))=\eta_{\text {out }}} \mathcal{D} \psi \\
& \cdot \exp \frac{1}{\hbar}\left(\frac{i}{4}(\psi(0), J \psi(0))+\int_{\mathcal{I}} \frac{1}{2}(\psi, d \psi)+\frac{i}{4}(\psi(1), J \psi(1))\right) .
\end{aligned}
$$

4.4.2. Path integral for the non-abelian one-dimensional Chern-Simons theory in the cyclic Whitney gauge. End of proof of Theorem 2. To obtain a path integral representation for the partition function of the one-dimensional Chern-Simons theory on an interval (61) we use the same strategy as in Sect. 4.4.1: we cut the interval into $N$ smaller intervals and then apply the map $\Phi$ (75). The new point here is that for small intervals we have to use the "heat kernel" approximation which gives an exact result only in the limit $N \rightarrow \infty$.

Applying $\Phi$ to $Z_{\mathcal{I}}(61)$, we have

$$
\begin{aligned}
\Phi\left(Z_{\mathcal{I}}\right)\left(\eta_{\text {out }}, \bar{\eta}_{\text {in }}\right)= & \int(i \hbar)^{-m} D \lambda \cdot e^{-(\lambda, \tilde{\psi})} \\
& \left.\cdot \Phi\left(\exp \left(-\frac{1}{2 \hbar}(\hat{\psi},[A, \hat{\psi}])+(\lambda, \hat{\psi})\right)\right) \eta_{\text {out }}, \bar{\eta}_{\text {in }}\right) \\
= & \int(i \hbar)^{-m} D \lambda \cdot e^{-(\lambda, \tilde{\psi})} \\
& \cdot \Phi\left(\left(\exp \left(-\frac{1}{2 \hbar N}(\hat{\psi},[A, \hat{\psi}])+\frac{1}{N}(\lambda, \hat{\psi})\right)\right)^{N}\right)\left(\eta_{\text {out }}, \bar{\eta}_{\text {in }}\right) \\
= & \int(i \hbar)^{-m} D \lambda \cdot e^{-(\lambda, \tilde{\psi})} \int\left(\prod_{k=1}^{N-1} \hbar^{m} D \eta_{k} D \bar{\eta}_{k+1}\right) \\
& \cdot \Phi\left(\exp \left(-\frac{1}{2 \hbar N}(\hat{\psi},[A, \hat{\psi}])+\frac{1}{N}(\lambda, \hat{\psi})\right)\right)\left(\eta_{\text {out }}, \bar{\eta}_{N}\right) \cdot e^{\frac{1}{\hbar}\left\langle\bar{\eta}_{N}, \eta_{N-1}\right\rangle} \\
& \ldots e^{\frac{1}{\hbar}\left\langle\bar{\eta}_{2}, \eta_{1}\right\rangle} \cdot \Phi\left(\exp \left(-\frac{1}{2 \hbar N}(\hat{\psi},[A, \hat{\psi}])+\frac{1}{N}(\lambda, \hat{\psi})\right)\right)\left(\eta_{1}, \bar{\eta}_{i n}\right) .
\end{aligned}
$$

Next, we need to evaluate the partition function for a small interval in the limit $N \rightarrow \infty$ :

$$
\begin{aligned}
& \Phi\left(\exp \left(-\frac{1}{2 \hbar N}(\hat{\psi},[A, \hat{\psi}])+\frac{1}{N}(\lambda, \hat{\psi})\right)\right)(\eta, \bar{\eta}) \\
& =\Phi\left(\hat{1}-\frac{1}{2 \hbar N}(\hat{\psi},[A, \hat{\psi}])+\frac{1}{N}(\lambda, \hat{\psi})+\mathcal{O}\left(\frac{1}{N^{2}}\right)\right)(\eta, \bar{\eta}) \\
& =e^{\frac{1}{\hbar}\langle\eta, \bar{\eta}\rangle}\left(1-\frac{1}{2 \hbar N}(\psi,[A, \psi])+\frac{1}{N}(\lambda, \psi)+\frac{i}{4 N} \operatorname{tr}\left(J \cdot \operatorname{ad}_{A}\right)+\mathcal{O}\left(\frac{1}{N^{2}}\right)\right) .
\end{aligned}
$$


Here $\psi$ is a linear combination of $\eta, \bar{\eta}$, prescribed by the choice of a complex structure $J$ (e.g. (97)); the term with a trace appeared due to the following identity:

$$
\Phi\left(\hat{\psi}^{a} \hat{\psi}^{b}\right)(\eta, \bar{\eta})=e^{\frac{1}{\hbar}\langle\eta, \bar{\eta}\rangle}\left(\psi^{a} \psi^{b}+\frac{\hbar}{2} \delta^{a b}+\frac{i \hbar}{2} J^{a b}\right) .
$$

Here the third term generates the trace term in (101). Substituting the "heat kernel" asymptotics (101) into (100), we get

$$
\begin{aligned}
\Phi\left(Z_{\mathcal{I}}\right)\left(\eta_{\text {out }}, \bar{\eta}_{\text {in }}\right) \\
=\int\left(\prod_{k=1}^{N-1} \hbar^{m} D \eta_{k} D \bar{\eta}_{k+1}\right) \int(i \hbar)^{-m} D \lambda \cdot e^{\left(\lambda, \frac{1}{N} \sum_{k=1}^{N} \psi_{k}-\tilde{\psi}\right)} \\
\quad \cdot e^{\frac{i}{4} \operatorname{tr}\left(J \cdot \mathrm{ad}_{A}\right)} \cdot e^{\frac{1}{\hbar}\left(\left\langle\eta_{\text {out }}, \bar{\eta}_{N}\right\rangle+\left\langle\bar{\eta}_{N}, \eta_{N-1}\right\rangle \cdots+\left\langle\bar{\eta}_{2}, \eta_{1}\right\rangle+\left\langle\eta_{1}, \bar{\eta}_{i n}\right\rangle\right)} \\
\quad \cdot e^{-\frac{1}{2 \hbar N} \sum_{k=1}^{N}\left(\psi_{k},\left[A, \psi_{k}\right]\right)}+\mathcal{O}\left(\frac{1}{N}\right) .
\end{aligned}
$$

Taking the limit $N \rightarrow \infty$, we obtain the following.

Proposition 3. The Chern-Simons partition function for an interval is given by the path integral:

$$
\begin{aligned}
\Phi\left(Z_{\mathcal{I}}\right)\left(\eta_{\text {out }}, \bar{\eta}_{\text {in }}\right)= & e^{\frac{i}{4} \operatorname{tr}\left(J \cdot \operatorname{ad}_{A}\right)} \int_{\overline{\bar{\pi}}(\psi(0))=\bar{\eta}_{\text {in }}, \pi(\psi(1))=\eta_{\text {out }}, \int_{\mathcal{I}} d \tau \psi(\tau)=\tilde{\psi}} \mathcal{D} \psi \\
& \cdot \exp \frac{1}{\hbar}\left(\int_{\mathcal{I}} \frac{1}{2}\left(\psi,\left(d+d \tau \cdot \operatorname{ad}_{A}\right) \psi\right)\right. \\
& \left.+\frac{i}{4}(\psi(0), J \psi(0))+\frac{i}{4}(\psi(1), J \psi(1))\right) .
\end{aligned}
$$

The conditional measure on paths $\psi(\tau)$ with a fixed holomorphic projection at $\tau=1$, a fixed anti-holomorphic projection at $\tau=0$ and with a fixed integral over $\tau$ in (103) is the $N \rightarrow \infty$ limit of the measure in (102).

Applying the concatenation formulae (76), (77) to (103), we obtain a path integral representation of the Chern-Simons partition function for one-dimensional simplicial complexes:

Corollary 2. For a triangulated interval $\Theta=\left[\mathfrak{p}_{\text {in }}, \mathfrak{p}_{2}\right] \cup\left[\mathfrak{p}_{2}, \mathfrak{p}_{3}\right] \cup \cdots \cup\left[\mathfrak{p}_{n}, \mathfrak{p}_{\text {out }}\right]$ we have

$$
\begin{aligned}
\Phi\left(Z_{\Theta}\right)\left(\eta_{\text {out }}, \bar{\eta}_{\text {in }}\right)= & e^{\sum_{k=1}^{n} \frac{i}{4} \operatorname{tr}\left(J \cdot \operatorname{ad}_{A_{k}}\right)} \int_{\bar{\pi}\left(\psi\left(\mathfrak{p}_{\text {in }}\right)\right)=\bar{\eta}_{\text {in }}, \pi\left(\psi\left(\mathfrak{p}_{\text {out }}\right)\right)=\eta_{\text {out }}, \int_{\mathfrak{p}_{k}}^{\mathfrak{p}_{k+1}} d \tau \psi(\tau)=\tilde{\psi}_{k}} \mathcal{D} \psi \\
& \cdot \exp \frac{1}{\hbar}\left(\int_{\mathcal{I}} \frac{1}{2}\left(\psi,\left(d+d \tau \cdot \operatorname{ad}_{A}\right) \psi\right)+\frac{i}{4}\left(\psi\left(\mathfrak{p}_{\text {in }}\right), J \psi\left(\mathfrak{p}_{\text {in }}\right)\right)\right. \\
& \left.+\frac{i}{4}\left(\psi\left(\mathfrak{p}_{\text {out }}\right), J \psi\left(\mathfrak{p}_{\text {out }}\right)\right)\right) .
\end{aligned}
$$

For a triangulated circle $\Xi_{n}=\left[\mathfrak{p}_{1}, \mathfrak{p}_{2}\right] \cup \cdots \cup\left[\mathfrak{p}_{n-1}, \mathfrak{p}_{n}\right] \cup\left[\mathfrak{p}_{n}, \mathfrak{p}_{1}\right]$, we obtain:

$$
Z_{\Xi_{n}}=e^{\sum_{k=1}^{n} \frac{i}{4} \operatorname{tr}\left(J \cdot \operatorname{ad}_{A_{k}}\right)} \int_{\int_{\mathfrak{p}_{k}}^{\mathfrak{p}_{k+1}} d \tau \psi(\tau)=\tilde{\psi}_{k}} \mathcal{D} \psi \cdot e^{\frac{1}{2 \hbar} \int_{\mathcal{I}}\left(\psi,\left(d+d \tau \cdot \mathrm{ad}_{A}\right) \psi\right)}
$$


Remark 27. Expression (105) returns us to the "naïve" path-integral (37) for the simplicial Chern-Simons on a circle, up to a somewhat puzzling factor $e^{\sum_{k} \frac{i}{4} \operatorname{tr}\left(J \cdot \mathrm{ad}_{A_{k}}\right)}$. The explanation is as follows: the path integral in (105) was obtained from the path integral with boundaries (104) by the concatenation formula (77). Hence, it is secretly using the normal ordering prescribed by the choice of a complex structure $J$ on $\mathfrak{g}$ (which dictates the regularization for the one-loop determinant in (105)). This implicit dependence on $J$ is exactly cancelled by the factor $e^{\sum_{k} \frac{i}{4} \operatorname{tr}\left(J \cdot \mathrm{ad}_{A_{k}}\right)}$ (indeed, we know that the left hand side of (105) is defined in terms of the Clifford algebra $C l(\mathfrak{g})$ and therefore cannot possibly depend on $J$ ). For the naïve path integral (37), we implicitly assumed the symmetric normal ordering by making a regularization (41) in our computation of the one-loop determinant (the important point is that $\theta(0)$ is a number, and not a matrix).

Path integral representation (105) returns us to the perturbative computation of Sect. 2.3 and thus finishes the proof of Theorem 2.

Remark 28. It is easy to compute (103) in the case of $A=0$ :

$$
\Phi\left(\left.Z_{\mathcal{I}}\right|_{A=0}\right)\left(\eta_{\text {out }}, \bar{\eta}_{\text {in }}\right)=2^{-m} e^{\frac{1}{\hbar}\left(\left\langle\eta_{\text {out }}, \bar{\eta}_{\text {in }}\right\rangle-2\left\langle\eta_{\text {out }}-\tilde{\eta}, \bar{\eta}_{\text {in }}-\overline{\tilde{\eta}}\right\rangle\right)},
$$

where $\tilde{\eta}, \overline{\tilde{\eta}}$ are holomorphic and anti-holomorphic components of the bulk field $\tilde{\psi}$. We can also write (106) as

$$
\Phi\left(\left.Z_{\mathcal{I}}\right|_{A=0}\right)\left(\eta_{\text {out }}, \bar{\eta}_{i n}\right)=2^{-m} e^{\frac{1}{\hbar}\left(\frac{i}{2}\left(\psi_{b d}, J \psi_{b d}\right)-i\left(\psi_{b d}-\tilde{\psi}, J\left(\psi_{b d}-\tilde{\psi}\right)\right)\right)},
$$

where $\psi_{b d}=\iota\left(\eta_{\text {out }}\right)+\bar{\imath}\left(\bar{\eta}_{\text {in }}\right)$ is the linear combination of boundary fields $\eta_{\text {out }}, \bar{\eta}_{i n}$.

\subsection{Simplicial action on an interval.}

Proposition 4. The path integral for the Chern-Simons partition function on an interval (103) is given by

$$
\begin{aligned}
\Phi\left(Z_{\mathcal{I}}(\tilde{\psi}, A)\right)\left(\eta_{\text {out }}, \bar{\eta}_{\text {in }}\right)= & \operatorname{det}_{\mathfrak{g}}^{1 / 2}\left(\frac{\sinh \frac{\operatorname{ad}_{A}}{2}}{\frac{\operatorname{ad}_{A}}{2}}\right) \cdot \operatorname{det}_{\mathfrak{g}}^{-1 / 2} M\left(\operatorname{ad}_{A}\right) \\
& \cdot \exp \frac{1}{\hbar}\left(\left\langle\eta_{\text {out }}, \bar{\eta}_{\text {in }}\right\rangle-\frac{1}{2}\left(\tilde{\psi}, \operatorname{ad}_{A} \tilde{\psi}\right)\right. \\
& \left.+\frac{1}{2}\left(\tilde{\eta}-\eta_{\text {out }} \overline{\tilde{\eta}}-\bar{\eta}_{\text {in }}\right) \cdot M\left(\operatorname{ad}_{A}\right) \cdot\left(\begin{array}{c}
\tilde{\eta}-\eta_{\text {out }} \\
\tilde{\eta}-\bar{\eta}_{\text {in }}
\end{array}\right)\right),
\end{aligned}
$$

where the bilinear form $M\left(\operatorname{ad}_{A}\right)$ in basis $(\eta, \bar{\eta})$ is represented by the block matrix

$$
M\left(\operatorname{ad}_{A}\right)=\left(\begin{array}{cc}
R_{-+} R_{++}^{-1} & -1-R_{--}+R_{-+} R_{++}^{-1} R_{+-} \\
1+R_{++}^{-1} & R_{++}^{-1} R_{+-}
\end{array}\right) .
$$

Here symbols $R_{ \pm \pm}$stand for blocks of $R\left(\mathrm{ad}_{A}\right)$ (defined by formula (31)) in the basis $(\eta, \bar{\eta})$ :

$$
R\left(\operatorname{ad}_{A}\right)=\left(\begin{array}{ll}
R_{++} & R_{+-} \\
R_{-+} & R_{--}
\end{array}\right)
$$


Proof. The path integral (103) is Gaussian with a critical point $\psi^{c r}(\tau)$ being the solution of

$$
\left(d+d \tau \operatorname{ad}_{A}\right) \psi^{c r}=\text { const }
$$

subject to conditions

$$
\begin{aligned}
\int d \tau \psi^{c r}(\tau) & =\tilde{\psi}, \\
\pi\left(\psi^{c r}(1)\right) & =\eta_{o u t}, \\
\bar{\pi}\left(\psi^{c r}(0)\right) & =\bar{\eta}_{i n} .
\end{aligned}
$$

Equation (110) together with (111) gives

$$
\psi^{c r}(\tau)=\tilde{\psi}+F\left(\operatorname{ad}_{A}, \tau\right)\left(\psi^{c r}(0)-\tilde{\psi}\right),
$$

where $F\left(\operatorname{ad}_{A}, \tau\right)$ is defined by (30). The value of the action (together with boundary terms) for this path is

$$
\begin{aligned}
& S\left(\psi^{c r}\right)+\text { boundary terms } \\
& =\underbrace{-\frac{1}{2}\left(\tilde{\psi}, \operatorname{ad}_{A} \tilde{\psi}\right)-\frac{1}{2}\left(\tilde{\psi}, \psi^{c r}(1)-\psi^{c r}(0)\right)}_{\frac{1}{2} \int\left(\psi^{c r}, d_{A} \psi^{c r}\right)}+\frac{1}{2}\left\langle\eta^{c r}(0), \bar{\eta}_{\text {in }}\right\rangle+\frac{1}{2}\left\langle\eta_{\text {out }}, \bar{\eta}^{c r}(1)\right\rangle .
\end{aligned}
$$

Boundary values of $\psi^{c r}$ can be found as follows: (114) implies that

$$
\psi^{c r}(1)=\left(1+R\left(\operatorname{ad}_{A}\right)\right) \tilde{\psi}-R\left(\operatorname{ad}_{A}\right) \psi^{c r}(0) .
$$

Solving this equation together with (112) and (113) in coordinates $(\eta, \bar{\eta})$, we obtain

$$
\left(\begin{array}{l}
\eta_{\text {out }} \\
\bar{\eta}^{c r}(1)
\end{array}\right)=\left(\begin{array}{cc}
1+R_{++} & R_{+-} \\
R_{-+} & 1+R_{--}
\end{array}\right)\left(\begin{array}{l}
\tilde{\eta} \\
\tilde{\tilde{\eta}}
\end{array}\right)-\left(\begin{array}{ll}
R_{++} & R_{+-} \\
R_{-+} & R_{--}
\end{array}\right)\left(\begin{array}{l}
\eta^{c r}(0) \\
\bar{\eta}_{\text {in }}
\end{array}\right) .
$$

Here $\eta^{c r}(0)$ and $\bar{\eta}^{c r}(1)$ are the unknowns. Solving (116), we get

$$
\begin{aligned}
& \eta^{c r}(0)=\eta_{\text {out }}+\left(1+R_{++}^{-1}\right)\left(\tilde{\eta}-\eta_{\text {out }}\right)+R_{++}^{-1} R_{+-}\left(\overline{\tilde{\eta}}-\bar{\eta}_{\text {in }}\right), \\
& \bar{\eta}^{c r}(1)=\bar{\eta}_{\text {in }}-R_{-+} R_{++}^{-1}\left(\tilde{\eta}-\eta_{\text {out }}\right)-\left(-1-R_{--}+R_{-+} R_{++}^{-1} R_{+-}\right)\left(\overline{\tilde{\eta}}-\bar{\eta}_{\text {in }}\right) .
\end{aligned}
$$

By substituting into (115), we obtain the exponential in (107).

The pre-exponential in (107) can be derived as follows. We know that it is a function of $A$ only (since it is a square root of a functional determinant of the operator ${ }^{13} d_{A}$ acting on functions with vanishing integral, vanishing holomorphic part at $\tau=1$ and vanishing anti-holomorphic part at $\tau=0$ ); let us denote it by $G(A)$. Closing the interval into a circle (by using (77)), we find

$$
\begin{gathered}
\int \hbar^{m} D \eta_{\text {out }} D \bar{\eta}_{\text {in }} e^{\frac{1}{\hbar}\left\langle\bar{\eta}_{\text {in }}, \eta_{\text {out }}\right\rangle} \cdot \Phi\left(Z_{\mathcal{I}}(\tilde{\psi}, A)\right)\left(\eta_{\text {out }}, \bar{\eta}_{\text {in }}\right) \\
=\operatorname{det}_{\mathfrak{g}}^{1 / 2} M\left(\operatorname{ad}_{A}\right) \cdot G(A) \cdot e^{-\frac{1}{2 \hbar}\left(\tilde{\psi}, \operatorname{ad}_{A} \tilde{\psi}\right)} .
\end{gathered}
$$

Comparing with the known result for a circle (13), we obtain the pre-exponential in (107).

\footnotetext{
13 More exactly, the determinant of a matrix of the bilinear form $\int\left(\bullet, d_{A} \bullet\right)$.
} 
Remark 29. In this computation, we neglected the factor of $e^{\frac{i}{4} \operatorname{tr}\left(J \cdot \mathrm{ad}_{A}\right)}$. If we did not, it would anyway be cancelled by the pre-exponential obtained by comparison with (13) (and this result is obtained by an explicit computation in the operator formalism in Sect. 3.1.3). by

We can define the simplicial Chern-Simons action for the interval $S_{\mathcal{I}}\left(\tilde{\psi}, A ; \eta_{\text {out }}, \bar{\eta}_{\text {in }}\right)$

$$
e^{\frac{1}{\hbar} S_{\mathcal{I}}\left(\tilde{\psi}, A ; \eta_{\text {out }}, \bar{\eta}_{\text {in }}\right)}=\Phi\left(Z_{\mathcal{I}}(\tilde{\psi}, A)\right)\left(\eta_{\text {out }}, \bar{\eta}_{\text {in }}\right)
$$

or explicitly

$$
\begin{aligned}
& S_{\mathcal{I}}\left(\tilde{\psi}, A ; \eta_{\text {out }}, \bar{\eta}_{\text {in }}\right) \\
& =\left\langle\eta_{\text {out }}, \bar{\eta}_{\text {in }}\right\rangle-\frac{1}{2}\left(\tilde{\psi}, \operatorname{ad}_{A} \tilde{\psi}\right)+\frac{1}{2}\left(\tilde{\eta}-\eta_{\text {out }} \overline{\tilde{\eta}}-\bar{\eta}_{\text {in }}\right) \cdot M\left(\operatorname{ad}_{A}\right) \cdot\left(\begin{array}{l}
\tilde{\eta}-\eta_{\text {out }} \\
\overline{\tilde{\eta}}-\bar{\eta}_{\text {in }}
\end{array}\right) \\
& \quad+\frac{\hbar}{2} \operatorname{tr}_{\mathfrak{g}} \log \left(\frac{\sinh \frac{\operatorname{ad}_{A}}{2}}{\frac{\operatorname{ad}_{A}}{2}}\right)-\frac{\hbar}{2} \operatorname{tr}_{\mathfrak{g}} \log M\left(\operatorname{ad}_{A}\right) .
\end{aligned}
$$

Remark 30. Expansion of (117) as a power series in $A$ starts as

$$
\begin{aligned}
S_{\mathcal{I}} & \left(\tilde{\psi}, A ; \eta_{\text {out }}, \bar{\eta}_{i n}\right) \\
= & \frac{i}{2}\left(\psi_{b d}, J \psi_{b d}\right)-i\left(\psi_{b d}-\tilde{\psi}, J\left(\psi_{b d}-\tilde{\psi}\right)\right)-\frac{1}{2}\left(\tilde{\psi}, \operatorname{ad}_{A} \tilde{\psi}\right) \\
& -\frac{1}{6}\left(\psi_{b d}-\tilde{\psi}, J \operatorname{ad}_{A} J\left(\psi_{b d}-\tilde{\psi}\right)\right)+\mathcal{O}\left(A^{2}\right) \\
& -\hbar m \log 2+\hbar \frac{i}{12} \operatorname{tr}\left(J \operatorname{ad}_{A}\right)+\mathcal{O}\left(\hbar A^{2}\right),
\end{aligned}
$$

where $\psi_{b d}=\iota\left(\eta_{\text {out }}\right)+\bar{\imath}\left(\bar{\eta}_{\text {in }}\right)$.

We can obtain the action for a simplicial complex $\Theta$ by gluing actions (117) for individual intervals using concatenation formulae (76), (77). E.g. for a triangulated interval $\Theta=\left[\mathfrak{p}_{\text {in }}, \mathfrak{p}_{2}\right] \cup\left[\mathfrak{p}_{2}, \mathfrak{p}_{3}\right] \cup \cdots \cup\left[\mathfrak{p}_{n}, \mathfrak{p}_{\text {out }}\right]$ we have

$$
\begin{aligned}
e^{\frac{1}{\hbar} S_{\Theta}\left(\tilde{\psi}_{1}, A_{1}, \ldots, \tilde{\psi}_{n}, A_{n} ; \eta_{\text {out }}, \bar{\eta}_{\text {in }}\right)=} & \int \prod_{k=1}^{n-1}\left(\hbar^{m} D \eta_{k} D \bar{\eta}_{k+1}\right) . \\
& \cdot \exp \frac{1}{\hbar}\left(S_{\mathcal{I}}\left(\tilde{\psi}_{n}, A_{n} ; \eta_{\text {out }}, \bar{\eta}_{n}\right)+\left\langle\bar{\eta}_{n}, \eta_{n-1}\right\rangle\right. \\
& \left.+\cdots+\left\langle\bar{\eta}_{2}, \eta_{1}\right\rangle+S_{\mathcal{I}}\left(\tilde{\psi}_{1}, A_{1} ; \eta_{1}, \bar{\eta}_{\text {in }}\right)\right) .
\end{aligned}
$$

For a triangulated circle $\Xi_{n}=\left[\mathfrak{p}_{1}, \mathfrak{p}_{2}\right] \cup \cdots \cup\left[\mathfrak{p}_{n-1}, \mathfrak{p}_{n}\right] \cup\left[\mathfrak{p}_{n}, \mathfrak{p}_{1}\right]$, we have

$$
\begin{aligned}
e^{\frac{1}{\hbar} S_{\Xi_{n}}\left(\tilde{\psi}_{1}, A_{1}, \ldots, \tilde{\psi}_{n}, A_{n}\right)}= & \int \prod_{k=1}^{n}\left(\hbar^{m} D \eta_{k} D \bar{\eta}_{k+1}\right) \\
& \cdot \exp \frac{1}{\hbar} \sum_{k=1}^{n}\left(S_{\mathcal{I}}\left(\tilde{\psi}_{k}, A_{k} ; \eta_{k}, \bar{\eta}_{k}\right)+\left\langle\bar{\eta}_{k+1}, \eta_{k}\right\rangle\right) .
\end{aligned}
$$


Remark 31. Looking at formulae (119), (120), it is tempting to identify $\left\langle\bar{\eta}_{k+1}, \eta_{k}\right\rangle$ as a simplicial action for the point $\mathfrak{p}_{k+1}$.

Remark 32. Formula (120) explains how the simplicially non-local expression (42) is produced from a simplicially local expression (the sum of contributions of individual intervals — the integrand in (120)). The key is integration over boundary fields $\left\{\eta_{k}, \bar{\eta}_{k}\right\}$.

Let us introduce the notation $f_{ \pm \pm \pm}^{p q r}$ for structure constants ${ }^{14}$ of $\mathfrak{g}$ in the basis $(\eta, \bar{\eta})$ :

$$
\begin{aligned}
\theta & =\frac{1}{6} f^{a b c} \psi^{a} \psi^{b} \psi^{c} \\
& =\frac{1}{6} f_{+++}^{p q r} \eta^{p} \eta^{q} \eta^{r}+\frac{1}{2} f_{++-}^{p q r} \eta^{p} \eta^{q} \bar{\eta}^{r}+\frac{1}{2} f_{+--}^{p q r} \eta^{p} \bar{\eta}^{q} \bar{\eta}^{r}+\frac{1}{6} f_{---}^{p q r} \bar{\eta}^{p} \bar{\eta}^{q} \bar{\eta}^{r}
\end{aligned}
$$

(this is the same $\theta$ as in (4) rewritten in holomorphic-antiholomorphic coordinates on $\Pi \mathfrak{g})$. Then, the quantum master equation (68) for the action (117) has the following form:

$$
\begin{aligned}
\hbar & \frac{\partial}{\partial \tilde{\psi}^{a}} \frac{\partial}{\partial A^{a}} e^{\frac{1}{\hbar} S_{\mathcal{I}}(\tilde{\psi}, A ; \eta, \bar{\eta})}+\frac{1}{\hbar}\left(\frac{1}{6} f_{+++}^{p q r} \eta^{p} \eta^{q} \eta^{r}+\frac{\hbar}{2} f_{++-}^{p q r} \eta^{p} \eta^{q} \frac{\partial}{\partial \eta^{r}}-\frac{\hbar}{2} f_{++-}^{p q q} \eta^{p}\right. \\
& \left.+\frac{\hbar^{2}}{2} f_{+--}^{p q r} \eta^{p} \frac{\partial}{\partial \eta^{q}} \frac{\partial}{\partial \eta^{r}}-\frac{\hbar^{2}}{2} f_{+--}^{p p r} \frac{\partial}{\partial \eta^{r}}+\frac{\hbar^{3}}{6} f_{---}^{p q r} \frac{\partial}{\partial \eta^{p}} \frac{\partial}{\partial \eta^{q}} \frac{\partial}{\partial \eta^{r}}\right) e^{\frac{1}{\hbar} S_{\mathcal{I}}(\tilde{\psi}, A ; \eta, \bar{\eta})} \\
& -e^{\frac{1}{\hbar} S_{\mathcal{I}}(\tilde{\psi}, A ; \eta, \bar{\eta})} \frac{1}{\hbar}\left(\frac{\hbar^{3}}{6} f_{+++}^{p q r} \frac{\overleftarrow{\partial}}{\partial \bar{\eta}^{p}} \frac{\overleftarrow{\partial}}{\partial \bar{\eta}^{q}} \frac{\overleftarrow{\partial}}{\partial \bar{\eta}^{r}}+\frac{\hbar^{2}}{2} f_{++-}^{p q r} \frac{\overleftarrow{\partial}}{\partial \bar{\eta}^{p}} \frac{\overleftarrow{\partial}}{\partial \bar{\eta}^{q}} \bar{\eta}^{r}-\frac{\hbar^{2}}{2} f_{++-}^{p q q} \frac{\overleftarrow{\partial}}{\partial \bar{\eta}^{p}}\right. \\
& \left.+\frac{\hbar}{2} f_{+--}^{p q r} \frac{\grave{\partial}}{\partial \bar{\eta} p} \bar{\eta}^{q} \bar{\eta}^{r}-\frac{\hbar}{2} f_{+--}^{p p r} \bar{\eta}^{r}+\frac{\hbar^{3}}{6} f_{---}^{p q r} \bar{\eta}^{p} \bar{\eta}^{q} \bar{\eta}^{r}\right)=0
\end{aligned}
$$

Remark 33. The one-dimensional $B F$ theory is a special case of the one-dimensional Chern-Simons theory where the complex polarization (78) is compatible with the Lie algebra structure on $\mathfrak{g}$. In more detail, let $\mathfrak{h}$ in (78) be a Lie subalgebra, and let the Lie algebra structure on $\mathfrak{g}$ be given by a semidirect product of $\mathfrak{h}$ with its coadjoint module $\overline{\mathfrak{h}}$ :

$$
\mathfrak{g}=\mathfrak{h} \ltimes \overline{\mathfrak{h}} .
$$

In this case, formula (117) for $S_{\mathcal{I}}$ simplifies: the block $R_{+-}$in (109) vanishes, and the matrix $M\left(\operatorname{ad}_{A}\right)(108)$ becomes

$$
M\left(\operatorname{ad}_{A}\right)=\left(\begin{array}{cc}
R_{-+} R_{++}^{-1} & -1-R_{--} \\
1+R_{++}^{-1} & 0
\end{array}\right), \quad \operatorname{det}_{\mathfrak{g}}^{1 / 2} M\left(\operatorname{ad}_{A}\right)=\operatorname{det}_{\mathfrak{h}}\left(1+R_{++}^{-1}\right)
$$

In (121), only the second term on the right hand side survives:

$$
\theta=\frac{1}{2} F_{p q}^{r} \eta^{p} \eta^{q} \bar{\eta}_{r}
$$

\footnotetext{
${ }^{14}$ Here we mean the structure constants of the cyclic operation $(\bullet,[\bullet, \bullet]): \wedge^{3} \mathfrak{g} \rightarrow \mathbb{R}$.
} 
(Here $F_{p q}^{r}$ are the structure constants of $\mathfrak{h}$; we distinguish between upper and lower indices to emphasize that we do not assume that $\mathfrak{h}$ comes with a pairing). So, the quantum master equation (122) is simplified:

$$
\begin{aligned}
& \hbar \frac{\partial}{\partial \tilde{\psi}^{a}} \frac{\partial}{\partial A^{a}} e^{\frac{1}{\hbar} S_{\mathcal{I}}(\tilde{\psi}, A ; \eta, \bar{\eta})}+\frac{1}{\hbar}\left(\frac{\hbar}{2} F_{p q}^{r} \eta^{p} \eta^{q} \frac{\partial}{\partial \eta_{r}}-\frac{\hbar}{2} F_{p q}^{q} \eta^{p}\right) e^{\frac{1}{\hbar} S_{\mathcal{I}}(\tilde{\psi}, A ; \eta, \bar{\eta})} \\
& -e^{\frac{1}{\hbar} S_{\mathcal{I}}(\tilde{\psi}, A ; \eta, \bar{\eta})} \frac{1}{\hbar}\left(\frac{\hbar^{2}}{2} F_{p q}^{r} \frac{\overleftarrow{\partial}}{\partial \bar{\eta}^{p}} \frac{\overleftarrow{\partial}}{\partial \bar{\eta}^{q}} \bar{\eta}_{r}-\frac{\hbar^{2}}{2} F_{p q}^{q} \frac{\overleftarrow{\partial}}{\partial \bar{\eta}^{p}}\right)=0 .
\end{aligned}
$$

(If in addition $\mathfrak{h}$ is unimodular, the last terms in brackets vanish.) Note that the result for $B F$ theory that we obtain from (117) cannot be directly compared to the result in [15] as the choice of gauge fixing is very different. ${ }^{15}$

Remark 34. Another interesting point about the $B F$ case is that $f^{a b c} f^{a b c}=0$. Hence, the operator $\delta^{\rho}: \mathcal{H}_{p t^{+}} \rightarrow \mathcal{H}_{p t^{+}}$becomes a coboundary operator. If we assume in addition that $\mathfrak{h}$ is unimodular, then $\left(\mathcal{H}_{p t^{+}}, \delta^{\rho}\right)$ can be identified with the Chevalley-Eilenberg complex of the Lie algebra $\mathfrak{h}$. Thus, the reduced space of states associated to a point (see Remark 25) is the Chevalley-Eilenberg cohomology of $\mathfrak{h}$ :

$$
\mathcal{H}_{p t^{+}}^{\mathrm{red}} \cong H_{C E}(\mathfrak{h}) \text {. }
$$

Therefore, the cohomology space

$$
H_{\mathrm{ad}_{\hat{\theta}}}(C l(\mathfrak{g})) \cong H_{\delta_{\mathcal{I}}^{\rho}}\left(\operatorname{End}\left(\mathcal{H}_{p t^{+}}\right)\right) \cong H_{C E}(\mathfrak{h}) \otimes\left(H_{C E}(\mathfrak{h})\right)^{*}
$$

becomes non-trivial. In this case, the partition function $Z_{\mathcal{I}}^{\circ}$ can be understood as an identity operator acting on the Chevalley-Eilenberg cohomology $H_{C E}(\mathfrak{h})$.

Remark 35. One can also view the one-dimensional version of the $B F$ theory with a cosmological term [6] as a special case of the one-dimensional Chern-Simons theory for $\mathfrak{g}=\mathfrak{h} \oplus \mathfrak{h}^{*}$, where $\mathfrak{h}$ is itself a quadratic Lie algebra, and the Lie algebra structure on $\mathfrak{g}$ is given by

$$
\theta=\frac{1}{2} F^{p q r} \eta^{p} \eta^{q} \bar{\eta}^{r}+\kappa \frac{1}{6} F^{p q r} \bar{\eta}^{p} \bar{\eta}^{q} \bar{\eta}^{r} .
$$

Here $F^{p q r}$ are the structure constants of $\mathfrak{h}$ (in an orthonormal basis) and the parameter $\kappa$ is the "cosmological constant". For Lie algebra $\mathfrak{g}$, we automatically have $f^{a b c} f^{a b c}=0$, and Remark 25 applies in this case.

Let us denote $\mathfrak{g}$ with Lie algebra structure defined by (125) by $\mathfrak{g}_{B F, \kappa}$. Then, onedimensional Chern-Simons theories with Lie algebras $\mathfrak{g}_{B F, \kappa}$ and $\mathfrak{h}$ are related, similarly to the 3-dimensional case [6]. In particular, for continuum action on the circle we have

$$
\begin{aligned}
& S_{\mathfrak{g}_{B F, \kappa}}(\underbrace{\iota(\eta)+\bar{\iota}(\bar{\eta})}_{\psi}, \underbrace{\iota(\mathbf{A})+\bar{\iota}(\overline{\mathbf{A}})}_{A}) \\
& =\frac{1}{2 \kappa}\left(S_{\mathfrak{h}}(\eta+\kappa \bar{\eta}, \mathbf{A}+\kappa \overline{\mathbf{A}})-S_{\mathfrak{h}}(\eta-\kappa \bar{\eta}, \mathbf{A}-\kappa \overline{\mathbf{A}})\right),
\end{aligned}
$$

15 Indeed, here we fix the field $A$ to be constant on the interval, and we fix the integral $\tilde{\psi}$ of field $\psi$ over the interval, and the holomorphic and anti-holomorphic projections of $\psi$ at the right and left end-points of the interval. The gauge used in [15] fixes $\pi(A)$ to be constant, $\bar{\pi}(A)$ to be a sum of delta-functions at the ends of the interval; and it fixes the values $\pi(\psi)$ at the ends of the interval and the integral for $\bar{\pi}(\psi)$. The latter gauge choice features better simplicial locality properties, but is only $\mathfrak{h}$-equivariant. 
where $\iota$ and $\bar{\iota}$ denote the embeddings of $\mathfrak{h}, \mathfrak{h}^{*}$ into $\mathfrak{g}_{B F, \kappa}$ and on the right hand side we implicitly use the isomorphism $\mathfrak{h} \cong \mathfrak{h}^{*}$ given by the pairing on $\mathfrak{h}$. Relation (126) implies the following relation for partition functions for the triangulated circle $\Xi_{n}$ for Lie algebras $\mathfrak{g}_{B F, \kappa}$ and $\mathfrak{h}$ :

$$
\begin{aligned}
& Z_{\mathfrak{g}_{B F, \kappa}, \Xi_{n}}\left(\left\{\iota\left(\eta_{k}\right)+\bar{\imath}\left(\bar{\eta}_{k}\right)\right\},\left\{\iota\left(\mathbf{A}_{k}\right)+\bar{\imath}\left(\overline{\mathbf{A}}_{k}\right)\right\} ; \hbar\right) \\
& =Z_{\mathfrak{h}, \Xi_{n}}\left(\left\{\eta_{k}+\kappa \bar{\eta}_{k}\right\},\left\{\mathbf{A}_{k}+\kappa \overline{\mathbf{A}}_{k}\right\} ; 2 \kappa \hbar\right) \cdot Z_{\mathfrak{h}, \Xi_{n}}\left(\left\{\eta_{k}-\kappa \bar{\eta}_{k}\right\},\left\{\mathbf{A}_{k}-\kappa \overline{\mathbf{A}}_{k}\right\} ;-2 \kappa \hbar\right) .
\end{aligned}
$$

Remark 36. Another special case of a one-dimensional Chern-Simons theory can be constructed from a Lie bialgebra $\mathfrak{h}$. Here we set $\mathfrak{g}=\mathfrak{h} \oplus \mathfrak{h}^{*}$ with the canonical pairing and with Lie algebra structure on $\mathfrak{g}$ defined by

$$
\theta=\frac{1}{2} F_{p q}^{r} \eta^{p} \eta^{q} \bar{\eta}_{r}+\frac{1}{2} G_{p}^{q r} \eta^{p} \bar{\eta}_{q} \bar{\eta}_{r}
$$

Here $F_{p q}^{r}$ and $G_{p}^{q r}$ are structure constants of the Lie bracket and co-bracket on $\mathfrak{h}$. This is a one-dimensional version of the Lie bialgebra $B F$ theory, cf. [14] (the underlying unimodular Lie bialgebra for continuum theory on the circle is $\mathfrak{h} \otimes \Omega^{\bullet}\left(\mathcal{S}^{1}\right)$ ). It does not seem to enjoy any particular simplifications with respect to the general case other than having a canonical complex polarization on $\mathfrak{g}$.

Remark 37. The odd third-order differential operator in variables $\tilde{\psi}, A, \eta_{\text {out }}, \bar{\eta}_{\text {in }}$ that appears in (122) endows the algebra of functions Fun $(\Pi \mathfrak{g} \oplus \mathfrak{g} \oplus \Pi \mathfrak{h} \oplus \Pi \overline{\mathfrak{h}})$ with a structure of homotopy BV algebra in the sense of Tamarkin-Tsygan [16]. In general, the same applies to Fun $\left(\mathcal{F}_{\Theta}^{\text {bulk }} \oplus(\Pi \mathfrak{h})^{\times i(\Theta)} \oplus(\Pi \overline{\mathfrak{h}})^{\times i(\Theta)}\right)$ for any 1-dimensional simplicial complex $\Theta$. If $\Theta$ has no boundary, this homotopy BV structure is strict.

Acknowledgements. We wish to thank Alberto Cattaneo and Andrei Losev for enlightening discussions on the subject. Research of A. A. was supported in part by the grants of the Swiss National Science Foundation number 200020-129609 and 200020-126817; P. M. acknowledges partial support by SNF Grant 200020-121640/1 and by RFBR Grants 08-01-00638, 09-01-12150.

\section{References}

1. Aleksandrov, M., Kontsevich, M., Schwarz, A., Zaboronsky, O.: The geometry of the master equation and topological quantum field theory. Int. J. Mod. Phys. A 12, 1405-1430 (1997)

2. Alekseev, A., Meinrenken, E.: Clifford algebras and the classical dynamical Yang-Baxter equation. Math. Res. Lett. 10(2-3), 253-268 (2003)

3. Berezin, F.: Covariant and contravariant symbols of operators. (Russian) Izv. Akad. Nauk SSSR Ser. Mat. 66, 1134-1167 (1972)

4. Berline, N., Getzler, E., Vergne, M.: Heat kernels and Dirac operators, Grundlehren der Mathematischen Wissenschaften, Vol. 298. New York: Springer-Verlag, 1992

5. Atiyah, M.: Topological quantum field theories. Publ. Math. Inst. Hautes Etudes Sci. 68, 175-186 (1989)

6. Cattaneo, A.S., Cotta-Ramusino, P., Froehlich, J., Martellini, M.: Topological BF theories in 3 and 4 dimensions. J. Math. Phys. 36, 6137-6160 (1995)

7. Cattaneo, A.S., Mnev, P.: Remarks on Chern-Simons invariants. Commun. in Math. Phys. 293(3), 803-836 (2010)

8. Cattaneo, A.S., Mnev, P., Reshetikhin, N.: Perturbative topological quantum field theory with boundary. In preparation

9. Faddeev, L.D., Slavnov, A.A.: Gauge fields: an introduction to quantum theory. Reading, MA: Addison, Wesley, 1988

10. Frenkel, E., Losev, A., Nekrasov, N.: Instantons beyond topological theory I. http://arxiv.org/abs/hep-th/ 0610149v1, 2006 
11. Granåker, J.: Unimodular L-infinity algebras, http://arxiv.org/abs/0803.1763v1 [math.QA], 2008

12. Kostant, B., Sternberg, S.: Symplectic reduction, BRS cohomology, and infinite-dimensional Clifford algebras. Ann. Phys. 176(1), 49-113 (1987)

13. Losev, A.: Lectures on topological quantum field theory. 2008

14. Merkulov, S.A.: Wheeled pro(p)file of Batalin-Vilkovisky formalism. Commun. Math. Phys. 295, 585638 (2010)

15. Mnev, P.: Notes on simplicial BF theory. Moscow Math. J. 9(2), 371-410 (2009); Discrete BF theory. http://arxiv.org/abs/0809.1160v2 [hep-th], 2008

16. Tamarkin, D., Tsygan, B.: Noncommutative differential calculus, homotopy BV algebras and formality conjectures. Methods Funct. Anal. Topology 6(2), 85-100 (2000)

17. Whitney, H.: Geometric integration theory. Princeton, NJ: Princeton University Press, 1957

18. Witten, E.: Supersymmetry and Morse theory. J. Diff. Geom. 17(4), 661-692 (1982)

Communicated by A. Kapustin 